\title{
Stable concordance of knots in 3-manifolds
}

\author{
RoB SCHNEIDERMAN
}

\begin{abstract}
Knots and links in 3-manifolds are studied by applying intersection invariants to singular concordances. The resulting link invariants generalize the Arf invariant, the mod 2 Sato-Levine invariants and Milnor's triple linking numbers. Besides fitting into a general theory of Whitney towers, these invariants provide obstructions to the existence of a singular concordance which can be homotoped to an embedding after stabilization by connected sums with $S^{2} \times S^{2}$. Results include classifications of stably slice links in orientable 3-manifolds, stable knot concordance in products of an orientable surface with the circle and stable link concordance for many links of null-homotopic knots in orientable 3-manifolds.
\end{abstract}

57M27; 57M99

\section{Introduction}

A generic homotopy of a knotted circle in a 3-manifold $M$ traces out a properly immersed annulus in the product $M \times I$ of $M$ with an interval. Knot invariants can be extracted from homotopy invariants of such a singular concordance by factoring out the contributions from self-homotopies of knots. For instance, the (relative) selflinking invariants of the author [28] are determined by the self-intersection numbers of immersed annuli in the group ring $\mathbb{Z}\left[\pi_{1} M\right]$, and are characterized as the complete obstruction to finding an "improved" singular concordance in the sense that all of its singularities are paired by Whitney disks. In this setting, we will describe how a generalization of the intersection invariant of the author and P Teichner [31] can be used to define "higher order" linking invariants which give obstructions to building a "higher order Whitney tower," meaning that intersections between annuli and the interiors of previously existing Whitney disks can be paired by a second layer of Whitney disks. As a corollary, we can in many cases determine which links can cobound a collection $A$ of properly immersed annuli in $M \times I$ such that $A$ is homotopic (rel boundary) to an embedding after stabilization by taking connected sums with copies of $S^{2} \times S^{2}$. If one end of such a stable concordance is the unlink then it can be capped off to form stable slice disks.

Besides detecting many infinite families of nonconcordant knots and links, the invariants defined here will give: 
- A complete characterization of links in orientable 3-manifolds which are stably slice.

- A classification of stable concordance for many links of null-homotopic knots in orientable 3-manifolds with torsion-free fundamental group.

- A classification of stable concordance for knots in products $F \times S^{1}$ of an orientable surface with the circle.

The approach taken here fits into the theory of Whitney towers in 4-manifolds (as developed by the author in joint work with $\mathrm{J}$ Conant and $\mathrm{P}$ Teichner $[8 ; 5 ; 6 ; 7 ; 30 ; 29$; $31 ; 32]$ ), but of equal interest in the current setting is how the topology of a 3-manifold interacts with its knot theory. This interaction is reflected in the indeterminacies in the invariants coming from self-homotopies of knots, which can be studied using classical 3-dimensional structure theorems, following the approaches of Kalfagianni [21] and Kirk-Livingston [22; 23]. It turns out that these indeterminacies depend only on lower order invariants which can often be easily computed in the 3-manifold.

The algebraic linking invariants of [28] are defined for all knots in a large family of orientable 3-manifolds. For a link of null-homotopic knots the invariants reduce to the usual equivariant linking form, but for knots in nontrivial (free) homotopy classes these invariants are relative invariants, which compare homotopic knots to a chosen "base knot." One of the main observations of [28] is that although such a choice of base knot is not canonical, there are certain preferred knots which "maximize" the geometric characterization of the invariants by "minimizing" the indeterminacies. These knots are called spherical knots in [28] because all their corresponding indeterminacies come from intersections between the knots and 2-spheres in the 3-manifold. (In general, knot self-homotopies project to tori in $M$ which can contribute indeterminacies even when $M$ is irreducible.) It is only with respect to spherical knots that the invariants of [28] classify order 1 Whitney concordance, ie give the complete obstruction to the existence of a singular concordance having all double points paired by Whitney disks.

In the present paper we take a more streamlined approach by directly prescribing the analogous "indeterminacy-minimizing" base knots (and links) which give the desired classification of stable concordance and equivalent characterizations in terms of Whitney towers. Note that we now have many more equivalence classes to examine; for instance in a non-simply connected 3-manifold there are infinitely many order 1 Whitney concordance classes of null-homotopic knots. We will describe how to choose appropriate knots so that the resulting indeterminacies only depend on the given equivalence class. These indeterminacies will be computable in terms of intersections between knots and 2-spheres, disks and annuli in $M$. 
The rest of this introduction will introduce enough terminology to state the main results, leaving detailed definitions and proofs to the body of the paper. The reader should note that there are some essentially cosmetic differences in notation between this paper and the closely related papers [28] and [31]. The notation used here is chosen to fit with the more recent literature on Whitney towers, and clarifications will be made as terminology is introduced. While a familiarity with [28] and [31] is certainly helpful, the present paper is to a large extent self-contained, including sketches of much relevant background material. In particular, properties of Whitney disks are described in detail and also well-illustrated in the examples.

We work in the smooth oriented category, however the techniques of this paper do not distinguish smooth from locally flat by the basic results on topological immersions in Freedman-Quinn [10]. Maps and manifolds will be assumed to be smooth except when otherwise noted, with orientations usually suppressed from notation. The unit interval $I=[0,1]$ will be reparametrized without mention. When possible we follow the convention of knot theory to confuse an immersion with its image.

\subsection{Intersection trees and stable embeddings}

The homotopy invariant denoted by $\tau$ in [31] was defined for immersed 2-spheres in 4-manifolds with vanishing Wall intersection invariant by counting "higher order" intersections between Whitney disks and the 2 -spheres. This invariant has since been shown to fit into an obstruction theory for immersed surfaces in 4-manifolds in terms of Whitney towers, where an order $n$ Whitney tower $\mathcal{W}$ has an order $n$ obstruction $\tau_{n}(\mathcal{W})$ which lives in an abelian group generated by trivalent trees decorated by fundamental group elements $[8 ; 5 ; 6 ; 7 ; 30 ; 29 ; 32]$. The vanishing of $\tau_{n}(\mathcal{W})$ implies the existence of an order $n+1$ Whitney tower on the underlying immersed surfaces. As discussed next and described in detail in Section 2, in this language $\tau_{0}(\mathcal{W})$ corresponds to Wall's intersection form, and $\tau_{1}(\mathcal{W})$ corresponds to the $\tau$ in [31].

Let $A=A_{1}, A_{2}, \ldots, A_{m}$ be a collection of properly immersed simply connected compact oriented surfaces in an oriented 4-manifold $X$. Such an $A$ is called a Whitney tower of order zero. Wall's intersection $\lambda\left(A_{i}, A_{j}\right)$ and self-intersection $\mu\left(A_{i}\right)$ invariants count signed elements of $\pi_{1} X$ associated to sheet-changing paths through the intersection points of $A$ (see Section 2.1 below and Freedman-Quinn [10]). The equivalent order zero intersection tree $\tau_{0}(A)$ takes values in a group $\mathcal{T}_{0}\left(\pi_{1} X\right)$ of decorated order zero trees (oriented edges labelled by elements of $\pi_{1} X$ ), and gives the complete obstruction to the existence of an order 1 Whitney tower $\mathcal{W}$ on $A$, where $\mathcal{W}$ consists of the $A_{i}$ together with a collection of Whitney disks pairing all the intersections and self-intersections among the $A_{i}$. 
For $A$ admitting such an order one Whitney tower $\mathcal{W}$, the order one intersection tree $\tau_{1}(A):=\tau_{1}(\mathcal{W})$ takes values in an abelian group $\mathcal{T}_{1}\left(\pi_{1} X\right) / \operatorname{INT}(A)$ generated by decorated order one trees (unitrivalent $\mathrm{Y}$-trees having a single trivalent vertex). Here each Y-tree $t_{p} \in \tau_{1}(\mathcal{W})$ corresponds to a transverse intersection point $p$ between an $A_{k}$ and the interior of a Whitney disk $W_{(i, j)}$ pairing intersections between $A_{i}$ and $A_{j}$. The univalent vertices of $t_{p}$ inherit the labels $i, j$ and $k$; and the oriented edges of $t_{p}$ are decorated by elements of $\pi_{1} X$ determined by sheet-changing paths through $W_{(i, j)}$ (see Figure 1 and Section 2.3 below). The target group includes intersection relations $\operatorname{INT}(A)$ which are determined by the order zero invariants $\lambda\left(A_{i}, S\right)$ where $S$ ranges over immersed 2-spheres representing generators of $\pi_{2} X$.
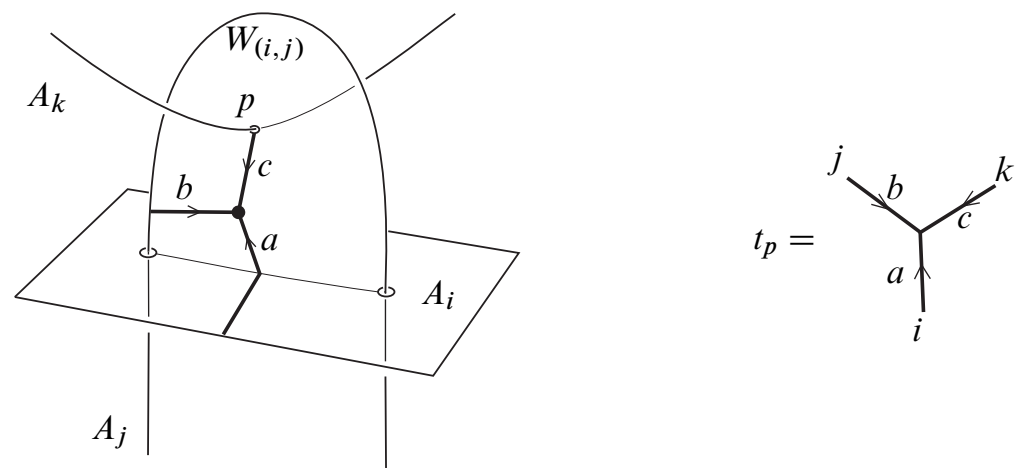

Figure 1: A Whitney disk $W_{(i, j)}$ pairing intersections between surface sheets $A_{i}$ and $A_{j}$ and the $\mathrm{Y}$-tree $t_{p}$ associated to an intersection point $p$ between the interior of $W_{(i, j)}$ and $A_{k}$.

As shown in [31], $\tau_{1}(A)$ does not depend on the choice of $\mathcal{W}$, and has the following geometric characterizations:

Theorem 1 [31] The following are equivalent:

(i) $\tau_{1}(A)$ vanishes.

(ii) $A$ is homotopic (rel boundary) to $A^{\prime}$ admitting an order 2 Whitney tower.

(iii) $A$ is homotopic (rel boundary) to $A^{\prime}$ admitting a height 1 Whitney tower.

Here an order 2 Whitney tower includes a second layer of Whitney disks pairing all intersections between the $A_{i}$ and the first layer of Whitney disks; and a height 1 Whitney tower is an order 1 Whitney tower such that all the interiors of the Whitney disks are disjoint from the $A_{i}$. This geometric characterization of $\tau_{1}$ corresponds to the fact that all the relations in the target are realizable by controlled manipulations 
of the Whitney tower. In the case where $A=A_{1}$ is a single component the group $\mathcal{T}_{1}\left(\pi_{1} X\right)$ is isomorphic to a quotient of the integral group ring $\mathbb{Z}\left[\pi_{1} X \times \pi_{1} X\right]$ by framing relations and an action of the symmetric group $\mathcal{S}_{3}$ on three elements; this is in complete analogy with the fact that Wall's self-intersection invariant $\mu$ (of order zero) takes values in $\mathbb{Z}\left[\pi_{1} X\right]$ modulo framing relations and an action of $\mathcal{S}_{2}$.

Definition 1 A collection $A$ of properly immersed surfaces $A_{i}$ in a 4-manifold $X$ can be stably embedded if for some $n$ the $A_{i}$ are homotopic (rel boundary) to pairwise disjoint embeddings in the connected sum of $X$ with $n$ copies of $S^{2} \times S^{2}$.

Here the sums are assumed to be taken along balls which are disjoint from $A$. Note that in the literature "stable embedding" usually means up to homology, rather than homotopy (see for example Bohr [1]).

The invariant $\tau_{1}(A)$ is an obstruction to homotoping $A$ to an embedding, and the following corollary gives a stable converse:

Corollary $1 \tau_{1}(A)$ vanishes if and only if $A$ can be stably embedded.

Corollary 1 follows from applying the "Norman trick" [26] to the height 1 Whitney tower in statement (iii) of Theorem 1, as explained in Section 2.5 below.

\subsection{Stably slice links}

A link $L$ in a 3-manifold $M$ is stably slice if the components of $L \subset M \times\{0\}$ bound a collection of properly immersed disks in $M \times I$ which can be stably embedded.

Note that a necessary condition for $L$ to be stably slice is that each component of $L$ is null-homotopic in $M$. As discussed later in Section 2.6, for a link $L$ of null-homotopic knots in $M$ the concordance invariant $\tau_{0}(L):=\tau_{0}(D) \in \mathcal{T}_{0}\left(\pi_{1} M\right)$, where $D$ is any collection of properly immersed disks in $M \times I$ bounded by $L \subset M \times\{0\}$, is the complete obstruction to $L$ bounding an order 1 Whitney tower on immersed disks in $M \times I$. For links with vanishing order zero obstruction, there is a similarly defined order 1 invariant:

Theorem 2 For any link of null-homotopic knots $L \subset M$ with vanishing $\tau_{0}(L)$, the order 1 intersection tree $\tau_{1}(L):=\tau_{1}(D) \in \mathcal{T}_{1}\left(\pi_{1} M\right)$ is a well-defined concordance invariant of $L$.

Theorem 2 is proved in Section 2.6. Note that in this setting the intersection relations $\operatorname{INT}(D)$ turn out to be trivial so $\tau_{1}(L)$ takes values in $\mathcal{T}_{1}\left(\pi_{1} M\right)$. We have the following characterization of stably slice links:

Corollary $2 L$ is stably slice if and only if $\tau_{1}(L)=0 \in \mathcal{T}_{1}\left(\pi_{1} M\right)$. Furthermore, each element of $\mathcal{T}_{1}\left(\pi_{1} M\right)$ is realized as $\tau_{1}(L)$ for some link $L$. 
The realization statement follows from iterating the construction in Figure 2.
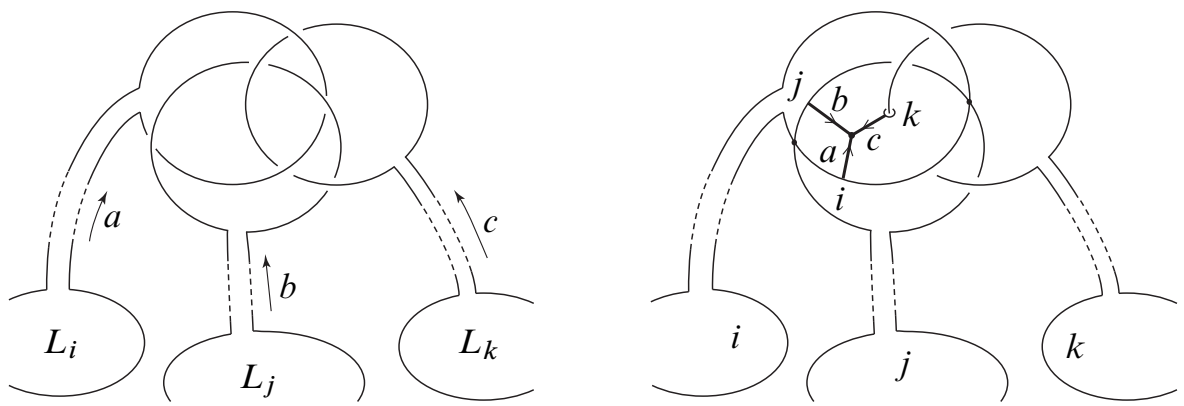

Figure 2: Tying the Borromean rings into an unlink along bands representing elements $a, b$ and $c$ in $\pi_{1} M$ yields a link $L$, with $\tau_{1}(L)$ represented by a Y-tree $t_{p}$ as in Figure 1. This is illustrated on the right, which shows $t_{p}$ in a Whitney disk for the canceling pair of intersection points in a null-homotopy of $L$.

If $\pi_{1} M$ is nontrivial and left-orderable, as many 3-manifold groups are (see BoyerRolfsen-Wiest [2]), then $\mathcal{T}_{1}\left(\pi_{1} M\right)$ is isomorphic to $\mathbb{Z}^{\infty} \oplus \mathbb{Z}_{2}^{\infty}$, and a normal form is given in Proposition 2.3.1 below. General methods for computing in $\mathcal{T}_{1}\left(\pi_{1} M\right)$ when $\pi_{1} M$ is not left-orderable would be useful.

Remark 1 (Finite type invariants) The reader familiar with finite type invariants will recognize that operation of Figure 2 can be effected by a Y-graph clasper or clover surgery $[16 ; 12]$. The links bounding order 1 Whitney towers are exactly the $\pi-$ algebraically split links which arise in the setting of finite type 3-manifold invariants proposed by Garoufalidis and Levine in [13]. The equivariant triple $\bar{\mu}$-invariants defined in [13], which characterize surgery equivalence of such links (in the sense of Levine [24]), correspond to the projection of $\tau_{1}(L)$ to the $\mathbb{Z}^{\infty}$ factors of $\mathcal{T}_{1}\left(\pi_{1} M\right)$ when $\pi_{1} M$ is left-orderable. Thus, in these cases the $\mathbb{Z}_{2}$ factors of $\tau_{1}$ detect infinite families of surgery equivalent links which are not (even stably) concordant.

Remark 2 (The Arf invariant) For a knot $K$ in a simply connected $M, \tau_{1}(K) \in$ $\mathcal{T}_{1}\left(\pi_{1} M\right) \cong \mathbb{Z}_{2}$ is just the $\operatorname{Arf}$ invariant $\operatorname{Arf}(K)$, which is known to be the complete obstruction to stably slicing $K[9 ; 25]$. For $K$ null-homotopic in general $M$, mapping $\pi_{1} M$ to the trivial group induces $\mathcal{T}_{1}\left(\pi_{1} M\right) \rightarrow \mathbb{Z}_{2}$ which takes $\tau_{1}(K)$ to $\operatorname{Arf}(K)$. It is easy to construct $K$ with trivial $\operatorname{Arf}(K)$ but nontrivial $\tau_{1}(K)$ by tying an even number of Borromean rings (with varying $\pi_{1} M$ decorations) into the unknot. Similar comments apply for the mod 2 reduction of the Sato-Levine invariant of a 2-component link of null-homotopic knots [27]. 
Remark 3 As noted in Section 2.6 below, for any link $L \subset M$ of null-homotopic knots with vanishing $\tau_{0}(L)$, we have $\tau_{1}(-L)=-\tau_{1}(L)$, where $-L$ denotes $L$ with reversed orientations on all components (fixing the orientation of $M$ ). So if $\pi_{1} M$ is left-orderable, then the $\mathbb{Z}^{\infty}$ factors in $\tau_{1}(L)$ represent obstructions to the existence of a (stable) concordance between $L$ and $-L$.

\subsection{Stable concordance of null-homotopic links}
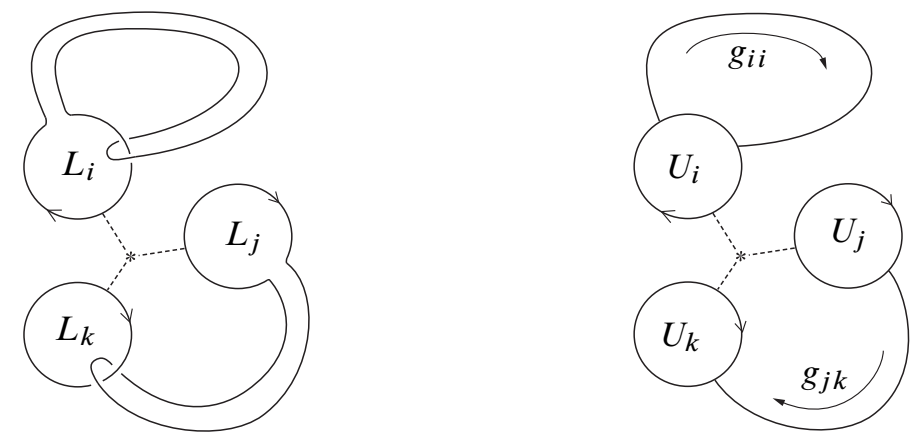

Figure 3: On the left a clasp link built from the unlink. On the right using the indicated guiding arcs.

Two links $L$ and $L^{\prime}$ in $M$ are stably concordant if there exists a collection $A$ of properly immersed annuli in $M \times I$, bounded by $L \subset M \times\{0\}$ and $L^{\prime} \subset M \times\{1\}$, such that $A$ can be stably embedded.

To classify stable concordance we will need to extend the definition of $\tau_{1}$ to properly immersed annuli supporting an order 1 Whitney tower in such a way that Theorem 1 and Corollary 1 still hold. Restricting our attention to links of null-homotopic knots corresponds to inessential annuli, that is, proper immersions of annuli $(A, \partial A) \rightarrow$ ( $X, \partial X)$ which take the circle factors of $A$ to the trivial element of $\pi_{1} X$. In this case, the new indeterminacies present in $\tau_{1}(A)$ correspond to the boundaries of the Whitney disks winding around the annulus, and are computable from $\partial A \subset \partial X$. In the setting of links in 3-manifolds, these new INT indeterminacies, and others coming from nonseparating 2-spheres, turn out to only depend on the lower order linking invariant $\tau_{0}(L)$, where the link $L \subset \partial A$ consists of the half of the boundary components of $A$ sitting in either end of $M \times I$.

For links of null-homotopic knots, order 1 Whitney concordance is classified by $\tau_{0}(L) \in \mathcal{T}_{0}\left(\pi_{1} M\right)$ (Section 4.3 below and [30]). Any element $z \in \mathcal{T}_{0}\left(\pi_{1} M\right)$ can be realized as $\tau_{0}\left(L_{z}\right)$ for a link $L_{z}$ created by adding clasps to the unlink, with the clasps guided by loops determining the group elements in a representative of $z$ (see Figure 3). 
Fixing such $L_{z}$, for any $L$ with $\tau_{0}(L)=z$ we define the relative order 1 intersection tree $\tau_{1}\left(L_{z}, L\right)$

$$
\tau_{1}\left(L_{z}, L\right):=\tau_{1}(A) \in \frac{\mathcal{T}_{1}\left(\pi_{1} M\right) / \operatorname{INT}(z)}{\Phi(z)}
$$

where $A$ is any singular concordance between $L_{z}$ and $L$ admitting an order 1 Whitney tower. Here, the intersection relations $\operatorname{INT}(z):=\operatorname{INT}(A)$ turn out to only depend on $z$; and the indeterminacy subgroup $\Phi(z)<\mathcal{T}_{1}\left(\pi_{1} M\right) / \operatorname{INT}(z)$, which is determined by order 1 intersections between 2-spheres and $L_{z}$, also turns out to only depend on $z$ (Lemma 3.2.1 and Section 3.3).

In irreducible 3-manifolds $\Phi(z)$ is trivial for all $z$, and in many 3-manifolds the quotient by $\Phi(z)$ is sufficient to account for all indeterminacies in the choice of $A$, so that $\tau_{1}\left(L_{z}, L\right)$ is a well-defined link concordance invariant for all $z$. However, as explained below in Section 5.2, slightly restricting $z$ will greatly expand the number of cases for which $\tau_{1}\left(L_{z}, L\right)$ is well-defined. For now, we observe that there is an action of the $m$-fold cartesian product $\Psi:=\left(\pi_{1} M\right)^{m}$ on $\mathcal{T}_{0}\left(\pi_{1} M\right)$ which corresponds to choices of basings of the $m$ components of $L$ in computing $\tau_{0}(L)$. The stabilizer $\Psi_{z}<\Psi$ of $z$ is called untwisted if all elements of $\Psi_{z}$ induce the trivial permutation of the elements of $\pi_{1} M$ in a representative of $z$, and twisted otherwise (see Section 4.5 below). This notion of twisting will be seen to correspond to Möbius bands and singular Seifert fibers, and in general a twisted stabilizer $\Psi_{z}$ can only occur for certain special forms of $z$.

As explained in Section 4, by choosing a clasp link $L_{z}$ in each order 1 Whitney concordance class we get the following classification theorem for many links of nullhomotopic knots:

Theorem 3 For any orientable 3-manifold $M$ with $\pi_{1} M$ torsion-free, and any $z \in \mathcal{T}_{0}\left(\pi_{1} M\right)$ with $\Psi_{z}$ untwisted, there exists a link $L_{z}$ in $M$ such that for any links $L$ and $L^{\prime}$ with $\tau_{0}(L)=\tau_{0}\left(L^{\prime}\right)=z \in \mathcal{T}_{0}\left(\pi_{1} M\right)$ the following are equivalent:

(i) $\tau_{1}\left(L_{z}, L\right)=\tau_{1}\left(L_{z}, L^{\prime}\right)$.

(ii) $L$ and $L^{\prime}$ are stably concordant.

(iii) $L$ and $L^{\prime}$ are order 2 Whitney concordant.

(iv) $L$ and $L^{\prime}$ are height 1 Whitney concordant.

Furthermore, for any such $L_{z}$ each element in the target group can be realized as $\tau_{1}\left(L_{z}, L\right)$ for some $L$. 
Here the equality in statement (i) is understood to be up to an action of $\Psi_{z}$ by left multiplication. By Proposition 5.2.1, $\Psi_{z}$ will be untwisted for all $z$ if $M$ contains no circle bundles over nonorientable surfaces with orientable total space, and no Seifert fibered submanifolds with singular fibers. The notions of order $n$ (resp. height $n$ ) Whitney concordance refer to the equivalence relations on links of cobounding immersed annuli supporting an order $n$ (resp. height $n$ ) Whitney tower in $M \times I$ (cf Theorem 1).

It follows that $\tau_{1}\left(L_{z}, \cdot\right)$ is a concordance invariant, and the effect of changing the choice of $L_{z}$ is computed in Section 5. The realization statement in Theorem 3 follows from repeatedly tying Borromean rings into $L_{z}$ (as in Figure 2), but the $\operatorname{INT}(z)$ and $\Phi(z)$ relations show that many such Borromean surgeries do not change the stable concordance class of $L_{z}$.

The target group for $\tau_{1}\left(L_{z}, \cdot\right)$ as in the setting of Theorem 5 is computed below in Section 3.1.1 for the case where $L_{z}$ is a knot in $S^{1} \times S^{2}$. Generating sets for the subgroups INT $(z)$ and $\Phi(z)$ in the general setting are given in Section 3, but the development of workable methods for computing the target quotients would be useful.

Note that the $\pi$-algebraically split links of [13] correspond to the case $z=0$ in Theorem 3; and, by taking $L_{0}$ to be the unlink, the relative invariant $\tau_{1}\left(L_{0}, L\right)$ reduces to the absolute invariant $\tau_{1}(L)$ (or $-\tau_{1}(L)$ depending on orientation conventions) since $\operatorname{INT}(0)$ and $\Phi(0)$ turn out to be trivial. It follows that any two $\pi$-algebraically split links $L$ and $L^{\prime}$ are stably concordant (and order 2 Whitney concordant and height 1 Whitney concordant) if and only if $\tau_{1}(L)=\tau_{1}\left(L^{\prime}\right) \in \mathcal{T}_{1}\left(\pi_{1} M\right)$.

The presence of twisted stabilizers can lead to additional indeterminacies coming from self-homotopies of $L_{z}$ as illustrated below in Section 5.3. A general method for dealing with such additional indeterminacies would be interesting, as would be an extension of $\tau_{1}$ and Theorem 3 to the case where $\pi_{1} M$ has torsion.

\subsection{Stable concordance of essential knots}

In the case where $M \cong F \times S^{1}$ is the product of an orientable surface $F \neq S^{2}$ with the circle, a stable concordance classification of knots is described by Theorem 5 at the end of Section 6, which extends the results of Theorem 3 to knots in arbitrary nontrivial free homotopy classes. The corresponding extension of $\tau_{1}$ to essential annuli requires two changes: To account for choices of paths, the decorations on Y-trees are taken in coset spaces of $\pi_{1} M$ by a cyclic group; and to account for choices of Whitney disk boundaries, the INT relations must be again modified. The resulting INT relations turn out to correspond to solutions of certain Baumslag-Solitar equations - which are highly restricted in 3-manifold groups - and can be computed in terms of lower order 
intersections among annuli, as explained in Section 6. Although these INT relations can be given explicitly, general methods for computing the target groups in this setting would be very useful.

\section{Intersection trees and stable embeddings}

This section recalls the definitions of the intersection invariants $\tau_{0}$ and $\tau_{1}$ for simply connected surfaces immersed in 4-manifolds, and sketches the proof that $\tau_{1}$ is a well-defined homotopy invariant - emphasizing aspects that will be relevant to the generalization of $\tau_{1}$ to immersed annuli. Proofs of Corollary 1 and Theorem 2 from the introduction are given in Sections 2.5 and 2.6. Additional background material on immersed surfaces in 4-manifolds can be found in Chapter 1 of Freedman-Quinn [10] (and absorbed from the examples below in Section 5).

\subsection{The order zero intersection tree $\tau_{0}$}

For $A=A_{1}, A_{2}, \ldots, A_{m}$ a collection of properly immersed simply connected oriented surfaces in an oriented 4-manifold $X$, Wall's hermitian intersection form $\lambda, \mu$ is defined as follows. The surfaces are perturbed into general position and equipped with whiskers (basepoints joined by a path to the basepoint of $X$ ). To each transverse intersection point $p \in A_{i} \cap A_{j}$ is associated a fundamental group element $g_{p} \in \pi_{1} X$ determined by a loop through $A_{i}$ and $A_{j}$ which changes sheets at $p$. Summing over all such intersection points, with the usual notion of the sign $\epsilon_{p}= \pm 1$, defines the intersection and self-intersection "numbers":

$$
\begin{aligned}
\lambda\left(A_{i}, A_{j}\right) & =\sum_{p \in A_{i} \cap A_{j}} \epsilon_{p} \cdot g_{p} \in \mathbb{Z}\left[\pi_{1} X\right] \\
\mu\left(A_{i}\right) & =\sum_{p \in A_{i} \cap A_{i}} \epsilon_{p} \cdot g_{p} \in \frac{\mathbb{Z}\left[\pi_{1} X\right]}{\mathbb{Z}[1] \oplus\{z-\bar{z}\}} .
\end{aligned}
$$

Modding out by the action of the involution $z \mapsto \bar{z}$ on $\mathbb{Z}\left[\pi_{1} X\right]$ induced by the map $g \mapsto$ $g^{-1}$ on $\pi_{1} X$ accounts for choices of orientations on the sheet-changing loops through the self-intersections of $A_{i}$. The relations killing $\mathbb{Z}[1]$ correspond to indeterminacies in the $\mu\left(A_{i}\right)$ due to local cusp homotopies which create self-intersections in $A_{i}$ whose double-point loops determine the trivial element $1 \in \pi_{1} X$.

Basic singularity theory shows that $\lambda\left(A_{i}, A_{j}\right)$ and $\mu\left(A_{i}\right)$ are invariant under homotopy of $A$ (rel boundary). Since the indeterminacy relations can be realized (by introducing local cusp homotopies and orienting double-point loops appropriately), the vanishing 
of $\lambda$ and $\mu$ implies that, after perhaps a homotopy (rel boundary), all intersection points of $A$ occur in canceling pairs having equal group elements and opposite signs. As described in detail below, each such canceling pair admits a Whitney disk.

If Whitney disks can be found that are all disjoint, with embedded interiors disjoint from $A$, and satisfying a normal framing condition described below, then such Whitney disks can be used to guide a homotopy of $A$ to an embedding. It can always be arranged that the Whitney disks are disjoint and framed, but in general they will have interior intersections with $A$. The obstruction theory of Whitney towers attempts to pair up these higher order intersections with higher order Whitney disks, and the resulting invariants associated to such a tower of Whitney disks naturally take values in groups generated by unitrivalent trees. These trees are associated to unpaired intersection points, and sit as subsets bifurcating down through the Whitney tower, with the trivalent vertices corresponding to Whitney disks, and the edges corresponding to sheet-changing paths.

In this language, the proper immersion $A$ is called a Whitney tower of order zero, and Wall's intersection form is an invariant of order zero (since there are no Whitney disks involved). In the notation of Whitney towers, the information contained in $\lambda$ and $\mu$ is expressed as the order zero intersection tree

$$
\tau_{0}(A):=\sum \epsilon_{p} \cdot t_{p} \in \mathcal{T}_{0}\left(\pi_{1} X\right)
$$

where each $t_{p}$ is an oriented edge (order zero trees have no trivalent vertices) which is decorated by $g_{p}$ and has endpoint vertices labelled by $i$ and $j$ for $p \in A_{i} \cap A_{j}$. We will use the notation $(g)_{i j}$ to denote an order zero tree which is decorated by $g$ and oriented from $i$ to $j$. The target $\mathcal{T}_{0}\left(\pi_{1} X\right)$ is the abelian group additively generated by such decorated edges modulo two kinds of relations: The orientation relations $(g)_{i j}=\left(g^{-1}\right)_{j i}$ reflect the Hermitian nature of the pairing $\lambda$ for $i \neq j$, and correspond for $i=j$ to the relations $z=\bar{z}$ in the self-intersection invariants $\mu\left(A_{i}\right)$ of the components of $A$. The framing relations (1) $)_{i i}=0$ correspond to the relations $\mathbb{Z}[1]=0$ in the $\mu\left(A_{i}\right)$ by killing any edges decorated by trivial group elements and having both vertices labeled by $i$. (With these framing relations one has a homotopy invariant; without the framing relations one has a regular homotopy invariant.)

Thus, $\tau_{0}(A)$ is exactly Wall's intersection form rewritten in Whitney tower notation, and the vanishing of $\tau_{0}(A)$ is equivalent to the existence of Whitney disks pairing all intersection points in $A$ (perhaps after performing some cusp homotopies). In the language of Whitney towers we say that such $A$ admits an order 1 Whitney tower. The Whitney disks in an order 1 Whitney tower are required to have pairwise disjointly embedded boundaries. The interiors of the Whitney disks are allowed to be immersed, 
and may intersect each other as well as $A$. All Whitney disks are required to satisfy a relative normal framing condition as described next.

\subsection{Framed Whitney disks}

Consider a canceling pair of intersections $p$ and $q$ between $A_{i}$ and $A_{j}$, with $g_{p}=$ $g_{q} \in \pi_{1} X$ and $\epsilon_{p}=-\epsilon_{q}$. The union of any pair of arcs, one in $A_{i}$ and the other in $A_{j}$, each connecting $p$ and $q$ (but avoiding all other singularities), forms a loop contractible in $X$, and any (immersed) disk $W$ bounded by such a loop is a Whitney disk. Since $p$ and $q$ have opposite signs, the restriction $v_{\partial W}$ of the normal disk bundle $v_{W}$ of $W$ in $X$ to $\partial W$ is the trivial bundle $S^{1} \times D^{2}$ (if the signs of $p$ and $q$ were equal, then we would get the nonorientable disk bundle over the circle).

The trivial $D^{2}$-bundle $v_{\partial W}$ has a nowhere-vanishing Whitney section constructed as follows (see Figure 4): Denote by $\partial_{i} W$ (resp. $\partial_{j} W$ ) the arc of $\partial W$ that lies in $A_{i}$ (resp. $A_{j}$ ). Over $\partial_{i} W$ choose a splitting of $v_{\partial W}$ given by a vector field $v_{i}^{t}$ tangent to $A_{i}$ and a vector field $v_{i}^{n}$ normal to both $W$ and $A_{i}$. Over $\partial_{j} W$ we have a similar splitting $\left\{v_{j}^{t}, v_{j}^{n}\right\}$ of $v_{\partial W}$, and we may arrange that these splittings coincide at $p$ and $q$ with $v_{i}^{t}=v_{j}^{n}$ and $v_{i}^{n}=v_{j}^{t}$. The Whitney section of $v_{\partial W}$ is constructed by taking $v_{i}^{t}$ over $\partial_{i} W$, and $v_{j}^{n}$ over $\partial_{j} W$. If this section of $v_{\partial W}$ can be extended to a nowhere-vanishing section of $v_{W}$, then following traditional terminology we say that $W$ is framed. (This terminology is only mildly abusive since $v_{W} \cong D^{2} \times D^{2}$ has a unique trivialization up to homotopy.)
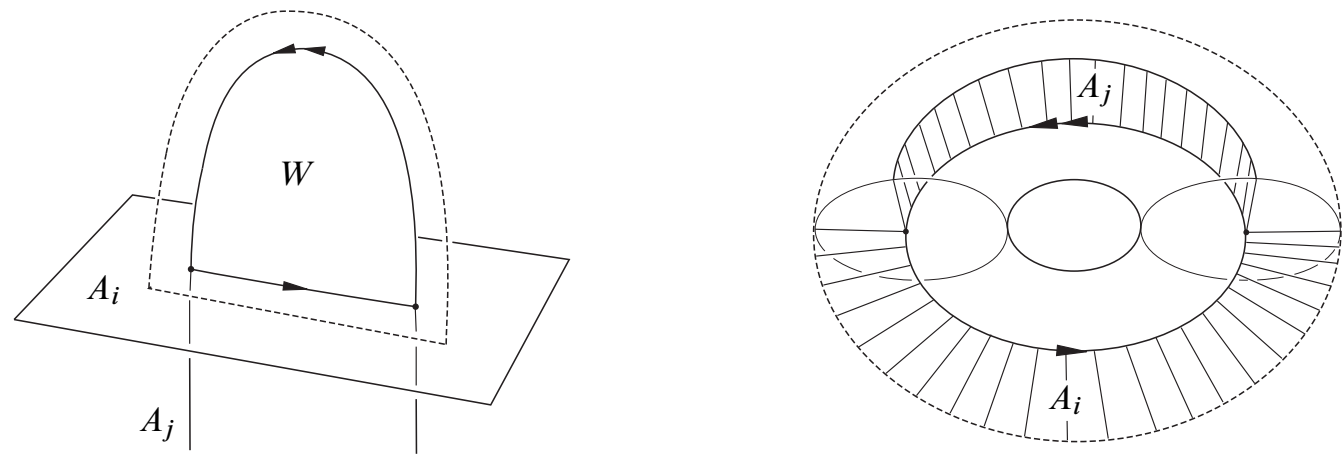

Figure 4: The Whitney section over the boundary of a framed Whitney disk is indicated by the dotted loop shown on the left for a clean Whitney disk $W$ in a 3-dimensional slice of 4-space, and on the right in the (pulled back) normal disk bundle over $\partial W$.

Fixing an orientation of $v_{W}$, the relative Euler number of the Whitney section gives an integer obstruction to extending the section over $W$ which is traditionally called 
the framing obstruction. This obstruction can always be killed at the cost of creating interior intersections between $W$ and $A_{i}$ (or $A_{j}$ ) by a boundary twisting modification of $W$ which changes the obstruction by \pm 1 . Also, the framing obstruction can be changed by \pm 2 at the cost of creating an interior self-intersection of $W$ by performing a cusp homotopy, called an interior twist. Boundary and interior twists are illustrated below in Figure 22 and Figure 19 of Section 5.

\subsection{The order 1 intersection tree $\tau_{1}$}

The invariant denoted $\tau$ in [31] corresponds in the general theory of Whitney towers [8; $5 ; 6 ; 7 ; 32]$ to the order 1 intersection tree $\tau_{1}$. For a collection $A$ of properly immersed simply connected surfaces with vanishing $\tau_{0}(A), \tau_{1}(A)$ is defined as follows. Choose an order 1 Whitney tower $\mathcal{W}$ on $A$, with fixed whiskers on the $A_{i}$ and on each of the Whitney disks in $\mathcal{W}$. To each intersection point $p \in W_{(i, j)} \cap A_{k}$ between $A_{k}$ and the interior of a Whitney disk $W_{(i, j)}$ pairing intersections between $A_{i}$ and $A_{j}$ is associated a trivalent Y-tree $t_{p}$ as illustrated in Figure 1. The univalent vertices are labelled by $i, j$ and $k$ and the oriented edges are decorated by elements $a, b$ and $c$ in $\pi_{1} X$ determined by sheet-changing loops through $W_{(i, j)}$ and the components of $A$ using the chosen whiskers, with the loop orientations corresponding to the edge orientations. Fixing (arbitrary) orientations on the Whitney disks in $\mathcal{W}$ associates a sign $\epsilon_{p}= \pm 1$ to each interior intersection point $p$, and determines a cyclic orientation at the trivalent vertex of the corresponding tree $t_{p}$ via the following convention: As illustrated in Figure 1, the tree $t_{p}$ sits as an embedded subset of $W_{(i, j)}$. The two edges of $t_{p}$ labelled $i$ and $j$ determine a "corner" of $W_{(i, j)}$ which does not contain the $k$-labelled edge, and the orientation of $t_{p}$ is taken to be that induced by $W_{(i, j)}$ if and only if this corner contains the positive intersection point between $A_{i}$ and $A_{j}$ that is paired by $W_{(i, j)}$.

(Due to differing orientation conventions there is a global sign difference between $\tau$ of [31] and the $\tau_{n}$ of more recent papers [8;32], including $\tau_{1}$ here.)

The order 1 intersection tree $\tau_{1}(\mathcal{W})$ is defined by summing the $t_{p}$ over all unpaired intersection points in $\mathcal{W}$ :

$$
\tau_{1}(\mathcal{W}):=\sum \epsilon_{p} \cdot t_{p} \in \frac{\mathcal{T}_{1}\left(\pi_{1} X\right)}{\operatorname{INT}(A)}
$$

The abelian group $\mathcal{T}_{1}\left(\pi_{1} X\right)$ is additively generated by the above described decorated Y-trees, modulo the relations shown in Figure 5. The $\operatorname{INT}(A)$ relations are shown in Figure 6. 

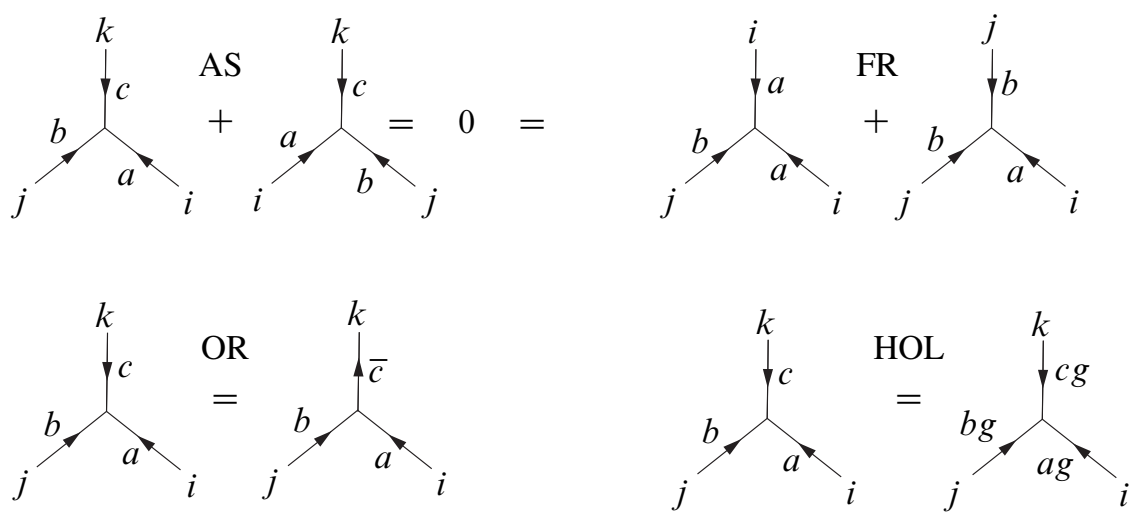

Figure 5: The AS antisymmetry, FR framing, OR orientation and HOL holonomy relations in $\mathcal{T}_{1}\left(\pi_{1} X\right)$, assuming a fixed planar orientation on the trivalent vertex of all Y-trees. Here $a, b, c$ and $g$ are elements of $\pi_{1} X$, with $\bar{c}=c^{-1}$. These edge labels can also be extended linearly to elements of $\mathbb{Z}\left[\pi_{1} X\right]$.
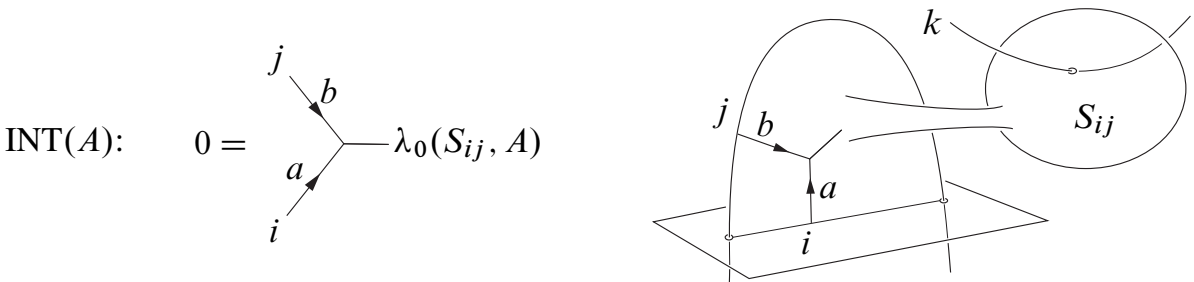

Figure 6: The INT(A) intersection relations. Here $S_{i j}$ varies over 2-spheres representing a generating set for $\pi_{2} X$, and the order zero invariant $\lambda_{0}\left(S_{i j}, A\right)$ counts only intersections between $S_{i j}$ and $A$, with the vertex label $k$ assigned to the intersections between $S_{i j}$ and $A_{k}$ (and edge labels from $\pi_{1} X$ ).

2.3.1 Notation and normal forms for $\mathcal{T}_{1}$ Note that the AS relation implies that the generators appearing in the FR relation are 2-torsion. By the OR edge-orientation relation, we can normalize all edges on generators of $\mathcal{T}_{1}\left(\pi_{1} X\right)$ to be oriented towards the trivalent vertex. We will use the cyclically oriented triple $(a, b, c)_{i j k}$, to denote such a Y-tree having univalent labels $i, j, k$, and corresponding group elements $a$, $b$, and $c$.

For fixed triples of univalent vertex labels, it will be convenient for us to represent the corresponding subgroups of $\mathcal{T}_{1}\left(\pi_{1} X\right)$ as quotients of the integral group ring $\mathbb{Z}\left[\pi_{1} X \times \pi_{1} X\right] \cong Z\left[\pi_{1} X\right] \times Z\left[\pi_{1} X\right]$ by using the HOL relation to normalize one edge decoration to the trivial group element $1 \in \pi_{1} X$ - for instance $(a, b, c)_{i j k}=$ 
$\left(1, b a^{-1}, c a^{-1}\right)_{i j k}$ - and summing group element decorations inside the parentheses. This corresponds to the notation used in [31] for $\tau=\tau_{1}$.

Assume that $\prec$ is a right-order on $\pi_{1} X-$ a strict total ordering of the elements of $\pi_{1} X$ such that if $g \prec h$ then $g f \prec h f-$ and that the components of $A$ (the univalent vertex labels) are ordered. Then we have the following normal form for $\mathcal{T}_{1}\left(\pi_{1} X\right)$ :

First consider a Y-tree whose univalent vertices all have the same label, and whose edge decorations are distinct group elements. Use the HOL relation to trivialize one edge decoration, and denote the resulting Y-tree by the ordered pair $(g, h):=(1, g, h)$, where we suppress the common univalent vertex label.

Such a generator $(g, h)$ is not involved in any FR relation (since $1 \neq g \neq h \neq 1$ ), and the orbit of $(g, h)$ under the AS and HOL relations in this notation is

$$
(g, h)=-\left(g^{-1}, h g^{-1}\right)=\left(h g^{-1}, g^{-1}\right)=-\left(g h^{-1}, h^{-1}\right)=\left(h^{-1}, g h^{-1}\right)=-(h, g) \text {. }
$$

Now one can check that for each of the possible relative orderings of $1, g$ and $h$ given by $\prec$, there is exactly one generator $(a, b)$ in the orbit of $(g, h)$ such that $1 \prec a \prec b$; for instance if $h \prec 1 \prec g$, then $\left(h^{-1}, g h^{-1}\right)$ satisfies $1 \prec h^{-1} \prec g h^{-1}$.

In the case where $g=h$, but still assuming all univalent labels are the same, then the generator is 2-torsion and we have the following orbit under the AS, HOL and FR relations:

$$
(g, g)=\left(g^{-1}, g^{-1}\right)=\left(g^{-1}, 1\right)=\left(1, g^{-1}\right)=(g, 1)=(1, g) .
$$

From each such orbit we get a unique generator $(1, a)$ such that $1 \preceq a$ (or a unique generator $(a, a)$ with $1 \preceq a)$.

This same approach works for other combinations of univalent labels, of course with different possible choices of convention. We state here the rest of a set of generators in normal form, written in ordered triple notation, with nondecreasing univalent labels (from left to right) which correspond to the cyclic orientation of the Y-tree:

$$
\begin{array}{lll}
(1, g, h)_{i j k} & \text { for distinct labels } \\
(1, g, h)_{i i j} & \text { with } 1 \prec g & \text { for labels } i<j, \\
(1, g, h)_{i j j} & \text { with } g \prec h & \text { for labels } i<j, \\
(1,1, g)_{i i j} & & \text { for labels } i<j .
\end{array}
$$

These last generators are 2-torsion.

This construction of a normal form, together with the observation that a (nontrivial) right-orderable group is infinite (it must be torsion free), gives the following proposition: 
Proposition 2.3.1 If $\pi_{1} X$ is nontrivial and right-orderable, then $\mathcal{T}_{1}\left(\pi_{1} X\right)$ is isomorphic to $\mathbb{Z}^{\infty} \oplus \mathbb{Z}_{2}^{\infty}$.

\subsection{Homotopy invariance of $\tau_{1}$}

We summarize here the proof in [31] that $\tau_{1}(A):=\tau_{1}(\mathcal{W})$ is a well-defined (regular) homotopy invariant of $A$, indicating the relevant points that will have to be addressed when we allow $A$ to have annular components. The proof proceeds by first checking that the value of $\tau_{1}(\mathcal{W})$ in $\mathcal{T}_{1}\left(\pi_{1} X\right) / \operatorname{INT}(A)$ does not depend on the choices in constructing the Whitney tower $\mathcal{W}$. Showing homotopy invariance is then reduced to checking invariance under finger moves.

With an eye towards our interest in the case $X=M \times I$, we will make the simplifying assumptions that $\pi_{1} X$ is torsion-free, and that $X$ is spin, ie, the second Stiefel-Whitney class $\omega_{2} X$ is trivial.

Independence of tree decorations and signs First of all, the group elements decorating the tree edges do not depend on the choices of sheet-changing loops since the $A_{i}$ and the Whitney disks are simply connected. The OR relations account for the orientation choices on the loops, which we will always assume to be oriented into the Whitney disks, so that all tree edges are oriented towards the trivalent vertex. The decorations do not depend on the choices of whiskers on the Whitney disks by the HOL relations.

The sign $\epsilon_{p}$ associated to each cyclically oriented $t_{p}$ is independent of the chosen Whitney disk orientations (for fixed orientations on the $A_{i}$ ) by the AS antisymmetry relations and the "positive corner" convention (Section 2.3).

Independence of Whitney disk interiors For fixed boundaries on the Whitney disks, the INT and FR relations guarantee that changing the Whitney disk interiors does not change $\tau_{1}(A)$; the idea is that such a change is given by intersections with 2 -spheres: Consider any Whitney disk $W=W_{(i, j)}$ in $\mathcal{W}$ pairing $p$ and $q$ in $A_{i} \cap A_{j}$. If $W^{\prime}$ is another Whitney disk with same boundary as $W$, then the union of $W$ and $W^{\prime}$ is a topological 2-sphere $S$, which may not be smooth along $\partial W=\partial W^{\prime}$. We may arrange (by a small isotopy and after perhaps performing some boundary twists on $W$ ) that along their common boundary the collars $C$ and $C^{\prime}$ of $W$ and $W^{\prime}$ point in opposite directions (Figure 7) so that $W \cup W^{\prime}$ is an immersed 2-sphere, still denoted $S$, which is oriented by the orientation of $W$ together with the opposite orientation of $W^{\prime}$. The boundary twists on $W$ ( $n$ twists into $A_{i}$ and $m$ twists into $A_{j}$ ) have changed $\tau_{1}(\mathcal{W})$ by $n(a, b, a)_{i j i}+m(a, b, b)_{i j j} \in \mathcal{T}_{1}\left(\pi_{1} X\right)-$ which is 2 -torsion by the AS relations 


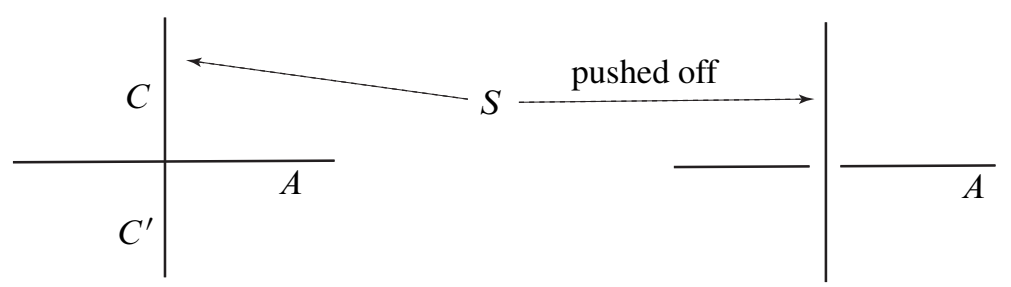

Figure 7: The 2-sphere $S$ formed as the union of two Whitney disks can be pushed off of $A$ near its equator - where the collars of the Whitney disks are joined along their boundaries.

- and have changed the framing obstruction of $W$ by $n+m \in \mathbb{Z}$. But by extending the Whitney section over the equator to a normal push-off of all of $S$ we see that $n+m$ is equal modulo 2 to $\omega_{2}(S) \in \mathbb{Z}_{2}$ which vanishes since $X$ is spin (note that any self-intersections of $S$ do not contribute to $\left.\omega_{2}(S)\right)$. So by the AS and FR relations $n(a, b, a)_{i j i}+m(a, b, b)_{i j j}=0 \in \mathcal{T}_{1}\left(\pi_{1} X\right)$.

Since the circle of intersection between $S$ and $A$ can be perturbed away without creating any new intersections (Figure 7), it follows that the change in $\tau_{1}(\mathcal{W})$ resulting from replacing $W$ by $W^{\prime}$ is exactly described by an $\operatorname{INT}(A)$ relation with $S=S_{i j}$.

Independence of Whitney disk boundaries To show that $\tau_{1}(\mathcal{W})$ does not depend on the choices of boundaries of the Whitney disks, for fixed pairings of the canceling singularities in $A$, it is convenient to weaken the definition of an order 1 Whitney tower by allowing transverse intersections among the boundaries of the Whitney disks (following [31] and 10.8 of [10]). The definition of $\tau_{1}$ is extended to such Whitney towers by assigning trees to the boundary intersections between Whitney disks in the following way (see Figure 8).
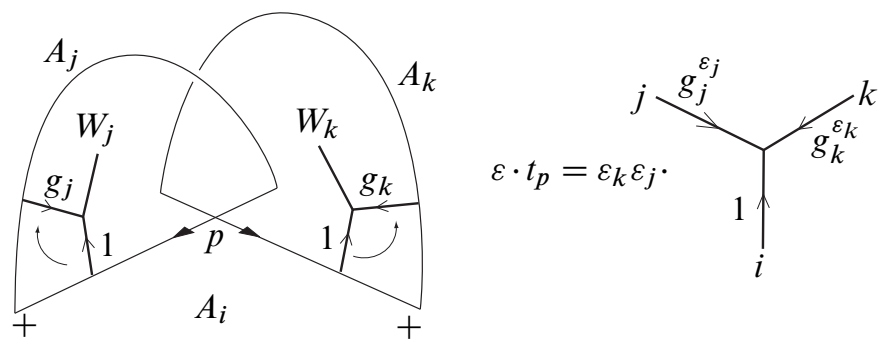

Figure 8: The signed tree $\epsilon_{p} \cdot t_{p}$ assigned to an intersection point $p \in$ $\partial_{\epsilon_{j}} W_{j} \cap \partial_{\epsilon_{k}} W_{k}$ between Whitney disk boundaries in the case $\epsilon_{k}=+=\epsilon_{j}$, with the orientations of $A_{i}$ and $t_{p}$ both the same as the plane of the paper 
Consider two Whitney disks, $W_{j}$ and $W_{k}$, pairing intersections that $A_{i}$ has with $A_{j}$ and $A_{k}$, respectively. Note that even if $W_{j}$ and $W_{k}$ have no interior intersections with any $A_{l}$, they still have naturally associated Y-trees, with only the "descending" edges and vertices decorated and labelled, as in the left hand side of Figure 8. Choose whiskers so that the edges labelled by $i$ are decorated by the trivial element $1 \in \pi_{1} X$, which then determines elements $g_{j}$ and $g_{k}$, respectively, decorating the edges labeled $j$ and $k$. The cyclic orientation of a Y-tree sitting in a Whitney disk $W$ corresponds to the orientation of $W$ by the "positive corner" convention described above in Section 2.3 , and induces an orientation of $\partial W$ (which corresponds to the convention that $\overrightarrow{\partial W}$ together with a second inward pointing vector give the orientation of $W$ ). We will use the notation $\partial_{+}$to indicate a Whitney disk boundary arc that is oriented towards its positive intersection point, and $\partial_{-}$for a Whitney disk boundary arc that is oriented towards its negative intersection point.

Now let $p \in \partial_{\epsilon_{j}} W_{j} \cap \partial_{\epsilon_{k}} W_{k}$ be a point such that the ordered pair of tangent vectors $\left(\overrightarrow{\partial_{\epsilon_{j}} W_{j}}, \overrightarrow{\partial_{\epsilon_{k}} W_{k}}\right)_{p}$ is equal to the orientation of $A_{i}$ at $p$. Define the tree $t_{p}$ associated to such a $p$ by

$$
\epsilon_{p} \cdot t_{p}:=\epsilon_{k} \epsilon_{j}\left(1, g_{k}^{\epsilon_{k}}, g_{j}^{\epsilon_{j}}\right)_{i k j}
$$

where $\epsilon \in\{+,-\} \hat{=}\{+1,-1\}$.

One can check that this definition of $t_{p}$ does not depend on the choices made. The extended version of $\tau_{1}(A)$ is defined by including such $t_{p}$ in the sum. Since all boundary intersections can be eliminated by finger moves which create interior intersections having the exact same trees (Figure 3 in [31]), this extended definition can always be reduced to the original one.

Properly interpreted, the formula assigning $t_{p}$ to $p \in \partial W_{j} \cap \partial W_{k}$ also works when $W_{j}=W_{k}$, including the case $i=j=k$. For instance, for $W$ pairing self-intersections of $A_{i}$, and $p \in \partial_{-} W \cap \partial_{+} W$ such that the orientation of $A_{i}$ is equal to $\left(\overrightarrow{\partial_{-} W}, \overrightarrow{\partial_{+} W}\right)_{p}$, then $\epsilon_{p} \cdot t_{p}=-\left(1, g, g^{-1}\right)_{i i i}$, where the $\mathrm{Y}$-tree associated to $W$ has decorations 1 and $g$ on the edges dual to the + and - boundary arcs respectively. (This formula will be used in Section 5 below.)

The proof of independence of Whitney disk boundaries now goes as follows. Since the components of $A$ are all simply connected, any configuration of Whitney disk boundaries can be achieved by a regular homotopy of (collars of) the Whitney disk boundaries, fixing the intersection points of $A$ (Clarification: we mean here that this regular homotopy is induced by a regular homotopy of the inverse images of the Whitney disk boundaries in the domain of $A$, and extends to a regular homotopy of collars of the Whitney disks in $X$ ). During such a homotopy, $\tau_{1}$ does not change since boundary 
intersections come and go in canceling pairs, or accompanied by a canceling interior intersection (when pushing over an intersection point of $A$; see Figure 5 in [31]).

Note that this step uses the fact that (the domains of) the components of $A$ are simply connected, and will have to be modified when we allow $A$ to have immersed annular components.

Independence of intersection pairings The independence of $\tau_{1}(\mathcal{W})$ on the choices of pairings of the intersections of $A$ follows easily from a construction pictured in Figure 6 of [31] (originally from 10.8 of [10]). In the presence of 2 -torsion in $\pi_{1} X$ there is also a subtle indeterminacy corresponding to the pairing of the inverse images of the self-intersections of the $A_{i}$ which was first explained in [33], and is covered by a more general INT relation [31] (which also covers the case where $X$ is not spin).

Homotopy invariance Having established the independence of $\tau_{1}(A):=\tau_{1}(\mathcal{W})$ on the choice of Whitney tower $\mathcal{W}$, homotopy invariance can be seen as follows. Up to isotopy, a generic homotopy (rel $\partial$ ) between surfaces in a 4-manifold is a sequence of finger moves (which create a pair of intersections), Whitney moves (which eliminate a pair of intersections), and local cusp homotopies (births and deaths of local selfintersections). In a regular homotopy it may be arranged that there are only finger moves and Whitney moves, and that the finger moves all occur before the Whitney moves. Since finger moves and Whitney moves are inverse to each other, it follows that if $A$ is regularly homotopic to $A^{\prime}$, then there is $A^{\prime \prime}$ which differs (up to isotopy) from each of $A$ and $A^{\prime}$ by only finger moves which create canceling intersections paired by local clean Whitney disks. Since these finger moves and their local clean Whitney disks can be assumed to be disjoint from all other Whitney disks in any Whitney tower on $A$ or $A^{\prime}$, it follows that $\tau_{1}(A)=\tau_{1}\left(A^{\prime \prime}\right)=\tau_{1}\left(A^{\prime}\right)$.

Geometric characterization That the vanishing of $\tau_{1}(A)$ leads to an order 2 and height 1 Whitney tower follows from the fact that all the relations in $\mathcal{T}_{1}\left(\pi_{1} X\right)$ can be realized by controlled manipulations of Whitney towers [31]. For instance, the FR and INT relations can be realized by creating clean Whitney disks, then twisting and tubing into 2-spheres. The new relations introduced later for the generalization of $\tau_{1}(A)$ which allows $A$ to have annular components will be similarly realizable.

\subsection{Proof of Corollary 1}

Proof The equivalence of statements (i) and (iii) in Theorem 1 is proved as Theorem 2 of [31] in the case where $A$ consists of a single connected component, but the exact same proof goes through for multiple components. Thus, the vanishing of $\tau_{1}(A)$ means 
that all the singularities of $A$ can be paired by (framed) Whitney disks whose interiors are disjoint from $A$. If the Whitney disk interiors are also disjointly embedded, then $A$ is homotopic to an embedding without any stabilization. Otherwise, it may be arranged by splitting the Whitney disks using finger moves that each Whitney disk is embedded, and has at most a single transverse interior intersection with some other Whitney disk. Each such intersection point $p \in W \cap W^{\prime}$ can be eliminated after taking a connected sum of $X$ with a copy of $S^{2} \times S^{2}$ by the Norman trick [26], which is illustrated schematically in Figure 9: If $S$ and $S^{\prime}$ are dual 2-sphere factors generating $\pi_{2}\left(S^{2} \times S^{2}\right)$, then $W$ can be tubed into $S$, and $p$ can be eliminated by tubing $W^{\prime}$ into $S^{\prime}$ along $W$. This operation can be done without creating any new singularities, and since $S$ and $S^{\prime}$ are both 0 -framed, the resulting Whitney disks are still framed. After one stabilization for each interior intersection between Whitney disks, the resulting disjointly embedded framed Whitney disks guide a homotopy of $A$ to an embedding in the connected sum of $X$ with (finitely many) copies of $S^{2} \times S^{2}$.
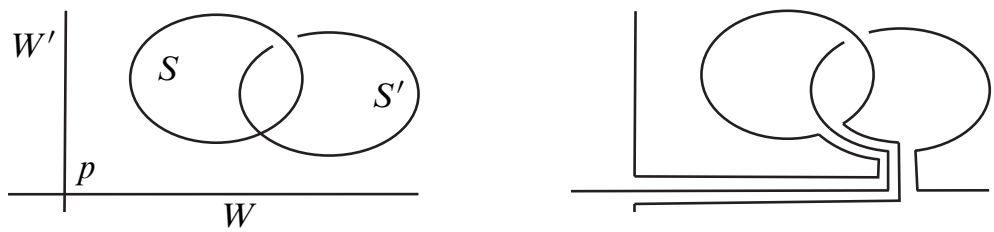

Figure 9: The Norman trick

On the other hand, if $A \rightarrow X$ is homotopic to an embedding in the connected sum of $X$ with copies of $S^{2} \times S^{2}$, then since $\tau_{1}(A)$ only depends on the homotopy class (rel boundary) of $A$, and since taking connected sums of $X$ with $S^{2} \times S^{2}$ does not change the $\operatorname{INT}(A)$ relations, it follows that $\tau_{1}(A)$ vanishes.

\subsection{Proof of Theorem 2}

This subsection gives a proof of the concordance invariance of the order 1 intersection tree $\tau_{1}(L)$ of a link (Theorem 2), which at the same time shows the concordance invariance of the order zero intersection tree $\tau_{0}(L)$.

Proof Consider an oriented $m$-component link $L$ of null-homotopic knots in an oriented 3-manifold $M$, with $D$ any collection of properly immersed disks in $M \times I$ bounded by $L \subset M \times\{0\}$. For $n=0$ or $n=1$, if $D$ admits an order $n$ Whitney tower, then we want to show that $\tau_{n}(L):=\tau_{n}(D)$ only depends on the concordance class of $L$. 
The key lemma is:

Lemma 2.6.1 [28] Any $m$ elements of $\pi_{2}(M \times I)$ are represented by $m$ embedded pairwise disjoint 2-spheres.

Proof A well known consequence of the Sphere Theorem of 3-manifold theory is that $\pi_{2} M$ is generated as a module over $\pi_{1} M$ by disjoint embeddings (the $2-$ spheres that decompose $M$ into prime factors, together with any spherical boundary components and cross-sections of any $S^{1} \times S^{2}$ factors; see Proposition 3.12 of Hatcher [17]). Tubing these generators together in $M \times I$ does not create any new intersections, so all elements of $\pi_{2}(M \times I)$ are represented by embeddings, which can be isotoped to be pairwise disjoint using the product structure.

We also need the following general properties of $\tau_{n}(A)$ for $A \leftrightarrow X$, which can be checked directly from the definitions: If $-A$ denotes a flip of orientation on (all components of) $A$, then $\tau_{0}(-A)=\tau_{0}(A)$ and $\tau_{1}(-A)=-\tau_{1}(A)$, where the orientation of the ambient 4-manifold $X$ is fixed. On the other hand, flipping the orientation of $X$ while fixing the orientation of $A$ has the effect of multiplying $\tau_{0}(A)$ by -1 , but preserves $\tau_{1}(A)$.

To see that $\tau_{n}(L)$ does not depend on the choice of $D$, let $D^{\prime}$ be another singular nullconcordance of $L$. Then the union $S$ of $D$ and $D^{\prime}$ along $L$, in two copies of $M \times I$ identified along $M$, determines $m$ elements of $\pi_{2}(M \times I) \cong \pi_{2} M$. The definition of $\tau_{n}(L):=\tau_{n}(D)$ depends on fixing a convention for the how the orientation of $L \subset M \times\{0\}$ induces an orientation of $D \rightarrow M \times I$. It follows that, after orienting $S$ in $M \times I$ (which requires reorienting one of the original copies of $M \times I$ and the corresponding singular null-concordance), we have

$$
0=\tau_{n}(S)= \pm\left(\tau_{n}(D)-\tau_{n}\left(D^{\prime}\right)\right)
$$

where the first equality comes from Lemma 2.6.1 and the homotopy invariance of $\tau_{n}$. It also follows from Lemma 2.6.1 that the $\operatorname{INT}(D)$ relations are trivial in the case $n=1$.

The sense in which $\tau_{n}(L)$ is a concordance invariant requires interpreting its value modulo the effect on $\mathcal{T}_{n}\left(\pi_{1} M\right)$ of whisker choices. This will be discussed in detail in Section 4. For now we observe that if $A$ is a concordance from $L^{\prime}$ to $L$ then, up to the change-of-whisker action, $\tau_{n}\left(L^{\prime}\right)=\tau_{n}\left(A \cup_{L} D\right)=\tau_{n}(D)=\tau_{n}(L)$ since $A$ has no singularities. This same argument shows that $\tau_{n}(L)$ is invariant under order $n+1$ Whitney concordance since then all singularities of $A$ of order less than $n+1$ occur in canceling pairs. 


\section{$3 \quad \tau_{1}$ for inessential annuli}

In this section we extend the definition of $\tau_{1}(A)$ to allow $A$ to have components which are inessential properly immersed annuli, meaning that the induced map $\mathbb{Z} \rightarrow \pi_{1} X$ on fundamental groups is trivial.

This generalized $\tau_{1}$ is illustrated by a pairing between links and 2 -spheres in $M$ described in Section 3.1 below, and computed in Section 3.2. These results are used in Section 3.3 to define the indeterminacy subgroup $\Phi(z)$ in the definition of the relative order 1 intersection tree $\tau_{1}\left(L_{z}, L\right)$ of Section 1.3. (The reader who is only interested in links in irreducible 3-manifolds can skip these three subsections.)

We continue to assume that $X$ is spin, and $\pi_{1} X$ is torsion-free.

Let $A \rightarrow X$ be a collection of properly immersed surfaces admitting an order 1 Whitney tower. We now allow the components of $A$ to include inessential annuli as well as 2-disks and 2-spheres. In order to extend the definition in Section 2.3 of $\tau_{1}(A)$ to such $A$ it will only be necessary to add some additional $\operatorname{INT}(A)$ relations.

Starting with the original Section 2.3, we proceed by examining the steps in the proof in Section 2.4 that $\tau_{1}(A)$ is a well-defined homotopy invariant.

First of all, note that $\tau_{0}(A)$ is still a well-defined homotopy invariant for such $A$ (with the vanishing of $\tau_{0}(A)$ equivalent to the existence of an order 1 Whitney tower on $A$ ) since in inessential annuli the elements of $\pi_{1} X$ associated to sheet-changing paths are still well-defined (because loops in $A$ which do not change sheets must represent $\left.1 \in \pi_{1} X\right)$. For the same reason the edge decorations from $\pi_{1} X$ on the trees in $\tau_{1}(A)$ are still well-defined.

The key step in Section 2.4 which relies on the assumption that the components of $A$ are simply connected is the argument showing independence of the choices of Whitney disk boundaries, which uses the fact that any two configurations of arcs with fixed endpoints in a simply connected surface are related by a homotopy (rel endpoints). This is no longer true when $A$ has annular components, and to account for the indeterminacies which correspond to changing the homotopy classes (rel endpoints) of the Whitney disk boundaries on annular components of $A$ we will include new $\operatorname{INT}(A)$ relations.

Consider any Whitney disk $W_{i j}$ pairing intersections between an annular component $A_{i}$ of $A$ and any component $A_{j}$ (including possibly $j=i$ ). Since $A_{i}$ is inessential, pushoffs of either component of $\partial A_{i}$ bound immersed disks in $X$. Let $D_{i j}$ be an immersed disk, with $\partial D_{i j}$ a parallel push-off of a component of $\partial A_{i}$ sitting in a collar on $\partial A_{i}$. The normal disk-bundle of $D_{i j}$ in $X$ has a nowhere vanishing section given by pushing tangentially along $A_{i}$, and we require that this section extends to a nowhere vanishing 
section over $D_{i j}$; this can always be arranged by boundary-twisting $D_{i j}$ if necessary. As illustrated in Figure 10, by half-tubing a collar of $W_{i j}$ into a collar of $D_{i j}$ along any embedded arc in $A_{i}$ we can change the homotopy class of $\partial W_{i j}$ (rel endpoints) in $A_{i}$ by \pm a generator of $\pi_{1} A_{i}$ (meaning that $\partial W_{i j}$ is band-summed into a loop which pulls back to a generator of the fundamental group of the domain of $A_{i}$ ).
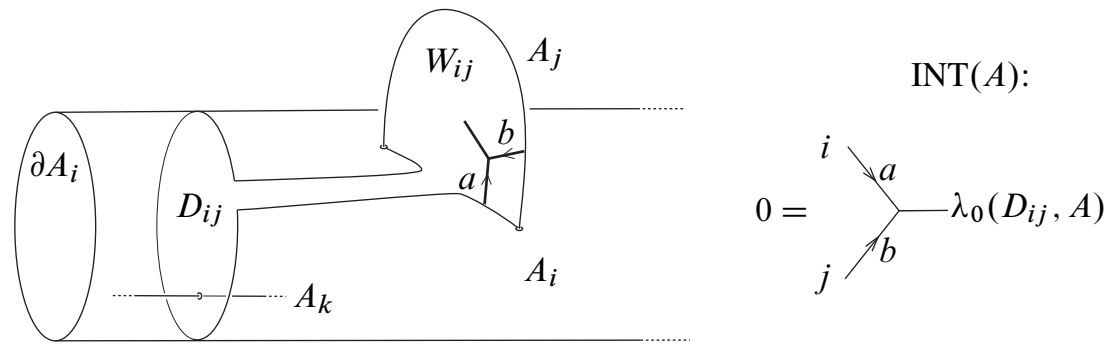

Figure 10: Changing the homotopy class in $A_{i}$ of the boundary of a Whitney disk leads to new $\operatorname{INT}(A)$ relations determined by the order zero intersection invariant $\lambda_{0}\left(D_{i j}, A\right)$, with the corresponding univalent labels taken from the components of $A$.

The framing requirement on $D_{i j}$ means that this operation preserves the framing on $W_{i j}$, and the resulting change in the contribution of $W_{i j}$ to $\tau_{1}(A)$ can be expressed as the relation illustrated in Figure 10, where $a$ and $b$ are the descending group elements for $W_{i j}$, and the order zero invariant $\lambda_{0}\left(D_{i j}, A\right)$ counts only intersections between $D_{i j}$ and all the $A_{k}$ (not intersections among the $A_{k}$ ), as was the case for the 2-spheres $S_{i j}$ in the original $\operatorname{INT}(A)$ relations of Figure 6.

By the proof of the independence of $\tau_{1}$ on the Whitney disk interiors (Section 2.4), using the usual INT( $A$ ) relations, this expression does not depend on the choice of $D_{i j}$ (or on the choice of the component of $\partial A_{i}$ ). Since the complement of any number of embedded arcs in an annulus is path-connected, this operation can be iterated any number of times on any number of Whitney disks, to get any choices of homotopy classes (rel endpoints) for the Whitney disk boundaries, so all the resulting indeterminacies are linear combinations of the expressions in Figure 10. By including these expressions into the $\operatorname{INT}(A)$ relations, and otherwise defining $\tau_{1}(A)$ as before, we get a well-defined homotopy invariant, with $A$ allowed to have inessential properly immersed annular components.

We remark that the original $\operatorname{INT}(A)$ relations are determined by computing order zero intersection invariants for a generating set for $\pi_{2} X$ (as a module over $\pi_{1} X$ ). These 
extended INT $(A)$ relations are determined by computing finitely many more order zero intersection invariants, which in our applications to links will essentially correspond to the order zero intersection tree of the link.

These new $\operatorname{INT}(A)$ relations can realized by performing finger-moves on $A$ to create clean Whitney disks with prescribed group elements, and then performing the above operation. Since the usual geometric manipulations of Whitney towers - such as "transferring" intersections between Whitney disks (Figure 10 in [31]) - also work for annular components, it follows that the geometric statements in Theorem 1 hold for this generalized version of $\tau_{1}(A)$.

Summarizing, we have:

Theorem 4 Theorem 1 and Corollary 1 also hold if any components of $A$ are inessential properly immersed annuli.

\subsection{The order 1 intersection pairing of links and spheres}

In this subsection we use Theorem 4 to define an order 1 intersection pairing between links and 2-spheres which determines the indeterminacy subgroup $\Phi(z)$ of Theorem 3.

Let $L$ be any link of null-homotopic knots, and $S$ be any immersed 2-sphere in $M$. Perturb $S$ into $M \times I$, and extend $L$ to the collection of embedded annuli $A=L \times I \subset$ $M \times I$. By Lemma 2.6.1, $S$ is homotopic to an embedding, and since the components of $L$ are null-homotopic it follows that $\tau_{0}(A, S)=0$. So we can define the pairing

$$
\tau_{1}(L, S):=\tau_{1}(A, S) \in \mathcal{T}_{1}\left(\pi_{1} M\right) / \operatorname{INT}(z)
$$

where we write $\operatorname{INT}(z)$ for $\operatorname{INT}(A, S)$, since (by Lemma 2.6.1 and the fact that the components of $L$ are null-homotopic) the only INT relations come from the boundary $L$, and are determined by $z=\tau_{0}(L)$ as follows. Using the ordering of the link components we can write $\tau_{0}(L)$ in a normal form:

$$
\tau_{0}(L)=z=\sum_{i \leq j} z_{i j}=\sum_{i \leq j} \sum_{p} e_{p} \cdot\left(g_{p}\right)_{i j},
$$

where the coefficients $e_{p}$ are nonzero integers, with the understanding that the indices $p$ depend on $i, j$-this is just collecting together all the intersections between $D_{i}$ and $D_{j}$ that have the same group element $g_{p}$. The group elements are assumed to be distinct, ie $\left(g_{p}\right)_{i j} \neq\left(g_{p^{\prime}}\right)_{i j}$ for $p \neq p^{\prime}$; and the only indeterminacy in this normal form for $z$ is in case $i=j$ we choose only one of $\left(g_{p}\right)_{i i}$ or $\left(g_{p}^{-1}\right)_{i i}$. 
Then each INT relation corresponding to a $2-$ disk $D_{i j}$ as in Figure 10, with the group element $a$ normalized to 1 by a HOL relation, can be written

$$
0=\left(1, g, \sum_{k \neq i} z_{k i}\right)_{i j k}+\left(1, g, z_{i i}+\overline{z_{i i}}\right)_{i j i}
$$

where the first term is determined by order zero linking between $L_{i}$ and the other components $L_{k}$ for $k \neq i$; and the second term is determined by order zero linking between $L_{i}$ and a 0 -parallel push-off $L_{i}^{\prime}$. Note that the second term is independent of the above mentioned indeterminacy in the normal form of $z$ (it corresponds to the relation $\lambda\left(D, D^{\prime}\right)=\mu(D)+\overline{\mu(D)}$ in Wall's intersection form for $D^{\prime}$ a zero-parallel push-off of $D$ - see the next example).

3.1.1 Example This subsubsection computes the pairing $\tau_{1}(L, S)$ in a case where $L$ is a knot, illustrating its relation to the study of $L$ up to stable concordance by defining the indeterminacy subgroup $\Phi(z)$ and target group of Theorem 3 in a simple example. It also serves as motivation and a warm-up for the more complicated computations in the subsequent two subsections.

Consider the case $M=S^{1} \times S^{2}$, with $\pi_{1} M \cong \mathbb{Z}$ generated multiplicatively by $x$. Let $x^{n} S$ denote a cross-sectional 2-sphere $S$ equipped with a whisker corresponding to $x^{n} \in \pi_{1} M$, and let $L$ be the clasp knot $K \subset M$ as in Figure 11 with $\tau_{0}(K)=$ $x \in \mathcal{T}_{0}(\langle x\rangle)$. (In this case $K$ is just a positive Whitehead double of a circle factor representing the generator $x$; by the "belt trick" there are two such Whitehead doubles up to isotopy.)
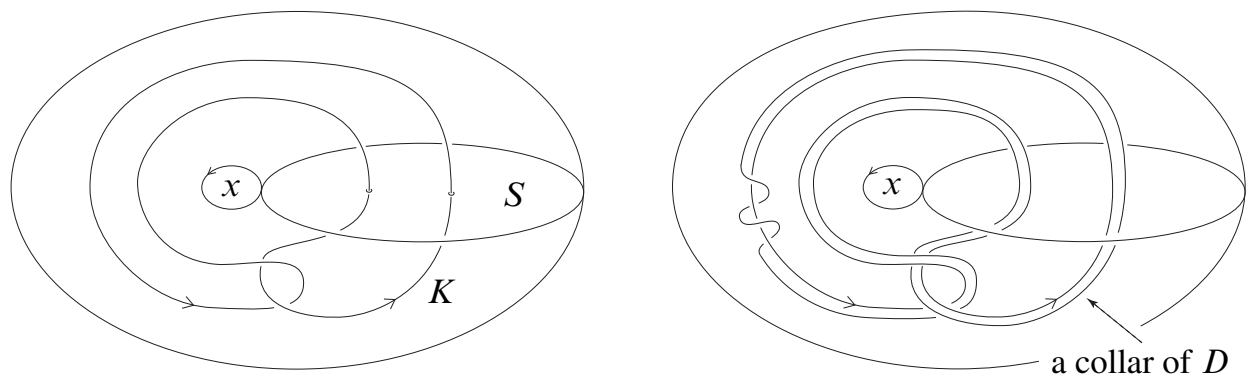

Figure 11: On the left, the clasp knot $K$ and (one hemisphere of) the crosssectional 2-sphere $S$ in $M=S^{1} \times S^{2}$ (with only $S^{1} \times D^{2}$ shown). On the right, a collar of a 0 -framed immersed 2-disk $D$ bounded by a parallel copy of $K$ in $K \times I$.

The target for $\tau_{1}\left(K, x^{n} S\right)$ is the quotient of $\mathcal{T}_{1}(\langle x\rangle)$, with univalent labels from $\{K, S\}$, by the intersection relations $\operatorname{INT}(K \times I, S)=\operatorname{INT}(x)$ which can be computed 
explicitly from intersecting a (framed) null-homotopy $D$ of a parallel copy of $K$ with $K \times I$ (crossing changes in the right hand side of Figure 11):

$$
\operatorname{INT}(x)=\left(1, x^{r}, x+x^{-1}-2\right)_{K j K}=\left(1, x^{r}, x+x^{-1}\right)_{K j K}=0
$$

for $j \in\{K, S\}$ and all $r \in \mathbb{Z}$. (Note that the contributions $\left(1, x^{r},-2\right)=-2\left(1, x^{r}, 1\right)$ from the two negative twists which ensure the framing of $D$ are killed by the AS relations.)

As illustrated in Figure 12, $K \times I$ and $x^{n} S$ support an order 1 Whitney tower $\mathcal{W}$ with a single Whitney disk, and

$$
\tau_{1}(\mathcal{W})=\left(1, x^{-1}, x^{n}\right)_{K K S}=\left(x, 1, x^{n+1}\right)_{K K S}=-\left(1, x, x^{n+1}\right)_{K K S}
$$

where the middle equality comes from the HOL relations, and the right-most equality comes from the AS relations.

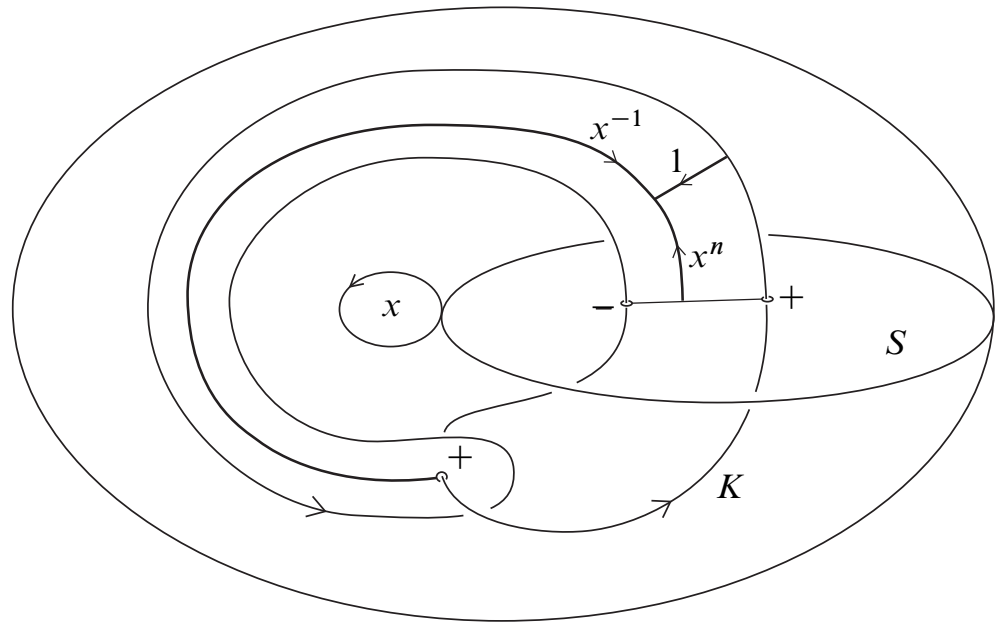

Figure 12: The order 1 Whitney tower on $K \times I$ and $x^{n} S$. (Whiskers are not shown.)

It follows that $\tau_{1}\left(K, x^{n} S\right)$ lies in the span of Y-trees having two univalent vertices labeled by $K$ and one by $S$. Using the HOL relation to trivialize one $K$-labeled edge decoration, we can write any such Y-tree as

$$
\left(x^{l}, x^{m}\right):=\left(1, x^{l}, x^{m}\right)_{K K S} \quad l, m \in \mathbb{Z} .
$$

By the AS and HOL relations we have

$$
\left(x^{l}, x^{m}\right)=-\left(x^{-l}, x^{m-l}\right),
$$


so the relevant subgroup of $\mathcal{T}_{1}(\langle x\rangle)$ is isomorphic to $\mathbb{Z}^{\infty} \oplus \mathbb{Z}_{2}^{\infty}$, with the $\mathbb{Z}$ factors generated by $\left(x^{l}, x^{m}\right)$, for $l \in \mathbb{N}$ and $m \in \mathbb{Z}$, and the $Z_{2}$ factors generated by $\left(1, x^{m}\right)$, for $m \in \mathbb{Z}$.

Now, in terms of these generators the relevant INT $(x)$ relations $\left(x+x^{-1}, x^{r}\right)=$ $\left(x, x^{r}\right)+\left(x^{-1}, x^{r}\right)=0$ give

$$
\left(x, x^{r}\right)=\left(x, x^{r+1}\right)
$$

for all $r \in \mathbb{Z}$ (using $\left(x^{-1}, x^{r}\right)=-\left(x, x^{r+1}\right)$ by HOL and AS).

So for all $n$ we have

$$
\tau_{1}\left(K, x^{n} S\right)=-\left(x, x^{n+1}\right)=-(x, x) \neq 0 \in \mathcal{T}_{1}(\langle x\rangle) / \operatorname{INT}(x) .
$$

The discussion so far has focused on computing $\tau_{1}\left(K, x^{n} S\right)$. Recall that the relevance to the knot theory of $K$ from our point of view is that $x^{n} S$ can be tubed into any singular concordance $A$ of $K$, and the corresponding indeterminacy in $\tau_{1}(A)$ lives in $\mathcal{T}_{1}(\langle x\rangle) / \operatorname{INT}(x)$ with all univalent labels corresponding to $K$. These indeterminacies are described algebraically by just replacing the $S$-labels with $K$-labels in $\tau_{1}\left(K, x^{n} S\right)$, which gives the indeterminacy subgroup $\Phi(x)<\mathcal{T}_{1}(\langle x\rangle) / \operatorname{INT}(x)$, as $x^{n}$ varies over $\pi_{1} M$ with the generator $S$ of $\pi_{2} M$ fixed.

Specifically, we can describe the quotient of $\mathcal{T}_{1}(\langle x\rangle) / \operatorname{INT}(x)$ by $\Phi(x)$ as follows:

Using the natural ordering of $\langle x\rangle \cong \mathbb{Z}$ with the chosen generator $x$, we can write the generators of $\mathcal{T}_{1}(\langle x\rangle)$ with all univalent vertices labelled by $K$ in normal form (as in Section 2.3.1 above):

$$
\mathcal{T}_{1}(\langle x\rangle)=\left\langle\left(x^{m}, x^{n}\right)\right\rangle_{1 \leq m<n} \oplus\left\langle\left(x^{r}, x^{r}\right)\right\rangle_{0 \leq r} \cong \mathbb{Z}^{\infty} \oplus \mathbb{Z}_{2}^{\infty} .
$$

Expressing the projected $\operatorname{INT}(x)$ relations $\left(x, x^{r}\right)+\left(x^{-1}, x^{r}\right)=0$ for $r \in \mathbb{Z}$ in the normal form generators gives the relations

$$
\left(x, x^{r}\right)=\left(x, x^{r+1}\right) \quad 1 \leq r \quad \text { and } \quad\left(x^{q}, x^{q+1}\right)=\left(x^{q+1}, x^{q+2}\right) \quad 0 \leq q
$$

which intersect when $r=2$ and $q=1$, so the $\operatorname{INT}(x)$ relations identify an infinite family of normal form generators.

Now the projected image $\Phi(x)$ of each of the pairings $\tau_{1}\left(K, x^{n} S\right)=-\left(x, x^{n+1}\right)=$ $-(x, x)$, for all $n$, is equal to (the inverse of) one of the generators identified by the INT relations, so the quotient of $\mathcal{T}_{1}(\langle x\rangle) / \operatorname{INT}(x)$ by $\Phi(x)$ is gotten by setting all the terms in Equation (1) equal to zero.

For example, by Theorem 3 any combination of Borromean surgeries on $K$ which correspond to linear combinations of terms in Equation (1) do not change the stable 
concordance class of $K$. On the other hand, there still exists $\mathbb{Z}^{\infty} \oplus \mathbb{Z}_{2}^{\infty}$ many distinct stable concordance classes of knots which are order 1 Whitney concordant to $K$, as detected by the generators $\left(x^{m}, x^{n}\right)$ with $|m-n| \geq 2$ and $m \neq 1$.

\subsection{Computing $\tau_{1}(L, S)$}

The computations of this subsection are used in the following subsection to describe the indeterminacy subgroup $\Phi(z)$ of Theorem 3 .

It follows from Theorem 4 that $\tau_{1}(L, S)$ is invariant under isotopy of $L$ and homotopy of $S$ in $M$. In (the proof of) Lemma 3.2.1 below we will see how $\tau_{1}(L, S)$ is invariant under order 1 Whitney concordance of $L$, and is completely determined by $S$ and $\tau_{0}(L)$.

In order to express $\tau_{1}(L, S)$ as a function of $S$ and $\tau_{0}(L)$ it will be useful to define a map $\sigma_{S}: \pi_{1} M \rightarrow \mathbb{Z}\left[\pi_{1} M\right]$ for each $S$ representing an element of $\pi_{2} M$. This will be done by defining the map on each generator of $\pi_{2} M$ as a $\pi_{1} M$-module, then extending linearly. We may ignore any generators which are spherical boundary components of $M$, since they clearly will have vanishing $\tau_{1}(L, S)$ for any $L$. The remaining generators consist of 2 -spheres that decompose $M$ into prime factors, and cross-sections of any $S^{1} \times S^{2}$ factors. The maps are defined slightly differently for each of these two types of spheres. (The first-time reader could at this point jump to Lemma 3.2.1, and then begin to absorb the definition of $\sigma_{S}$ during the subsequent proof.)

3.2.1 Separating spheres Let $S$ be an embedded separating sphere giving a connected sum decomposition of $M$ as $M=M^{\prime} \sharp_{S} M^{\prime \prime}$. Any $g \in \pi_{1} M$ can be written $g=h_{0} \prod_{l=1}^{n} g_{l} h_{l}$, where the $g_{l}$ are carried by $M^{\prime}$ and the $h_{l}$ are carried by $M^{\prime \prime}$. Denote by $\beta_{q}:=h_{q} \prod_{l=q+1}^{n} g_{l} h_{l}$, with $\beta_{n}=h_{n}$, and define $\sigma_{S}(g)$ by

$$
\sigma_{S}(g):=\sum_{q=1}^{n}\left(g_{q}-1\right) \beta_{q} \in \mathbb{Z}\left[\pi_{1} M\right] .
$$

Thus, here $\sigma_{S}(g)$ is roughly an alternating sum of "tails" of $g$. For example, in the case $n=2$ we have

$$
\sigma_{S}: g=h_{0} g_{1} h_{1} g_{2} h_{2} \mapsto\left(g_{1}-1\right)\left(h_{1} g_{2} h_{2}\right)+\left(g_{2}-1\right) h_{2} .
$$

3.2.2 Nonseparating spheres The map $\sigma_{S}$ is similar but slightly more complicated for nonseparating spheres. Let $S$ be a spherical section of the $S^{1} \times S^{2}$ factor in a connected sum decomposition of $M$ as $M=\left(S^{1} \times S^{2}\right) \sharp M^{\prime}$. Any $g \in \pi_{1} M$ can 
be written $g=h_{0} \prod_{l=1}^{n} x^{\epsilon_{l} r_{l}} h_{l}$, where $x$ is represented by the circle in the $S^{1} \times S^{2}$ factor, with $\epsilon_{l}= \pm 1$ and the $r_{l}$ positive integers, and the $h_{l}$ are carried by $M^{\prime}$. Let $\beta_{q}:=h_{q} \prod_{l=q+1}^{n} x^{\epsilon_{l} r_{l}} h_{l}$, with $\beta_{n}=h_{n}$, and define $\sigma_{S}(g)$ by

$$
\sigma_{S}(g):=\sum_{q=1}^{n} \epsilon_{q}\left(\sum_{l=1}^{r_{q}} x^{\epsilon_{q}\left(l+\left(\epsilon_{q}-1\right) / 2\right)}\right) \beta_{q} \in \mathbb{Z}\left[\pi_{1} M\right] .
$$

For example, in the case $n=2, r_{1}=3=r_{2}, \epsilon_{1}=1=-\epsilon_{2}$ we have

$$
\sigma_{S}: g=h_{0} x^{3} h_{1} x^{-3} h_{2} \mapsto\left(x^{3}+x^{2}+x\right) h_{1} x^{-3} h_{2}-\left(1+x^{-1}+x^{-2}\right) h_{2} .
$$

So for separating spheres $\sigma_{S}(g)$ is again a sum of tails of $g$, but multiplied by the indicated unit-coefficient polynomials in $x$ or $x^{-1}$.

3.2.3 Having defined $\sigma_{S}$ on generators, the definition for arbitrary elements of $\pi_{2} M$ is gotten by extending linearly: $\sigma_{\left(a S \pm a^{\prime} S^{\prime}\right)}(g):=a \sigma_{S}(g) \pm a^{\prime} \sigma_{S^{\prime}}(g)$.

Lemma 3.2.1 Let $S$ be any immersed 2-sphere in $M$. For any $z \in \mathcal{T}_{0}\left(\pi_{1} M\right)$, and any link $L \subset M$ of null-homotopic knots with $\tau_{0}(L)=z$,

$$
\tau_{1}(L, S)=\sum_{i \leq j} \sum_{p} e_{p} \cdot\left(g_{p}, 1, \sigma_{S}\left(g_{p}\right)\right)_{i j S} \in \mathcal{T}_{1}\left(\pi_{1} M\right) / \operatorname{INT}(z)
$$

where $z=\sum_{i \leq j} \sum_{p} e_{p} \cdot\left(g_{p}\right)_{i j}$

Here $z$ is written in normal form as in Section 3.1 above.

Proof First of all, we claim that if $L^{\prime}$ is any other link with $\tau_{0}\left(L^{\prime}\right)=z$, then $\tau_{1}\left(L^{\prime}, S\right)=\tau_{1}(L, S)$ :

As mentioned above in Section 3.1, the $\operatorname{INT}\left(L^{\prime}\right)$ and $\operatorname{INT}(L)$ relations coincide, since they only depend $z$. The equality of $\tau_{0}$ implies that there exists a homotopy from $L^{\prime}$ to $L$ whose trace is a collection of properly immersed annuli $A^{\prime} \rightarrow M \times I$ supporting an order 1 Whitney tower, and connecting the link components in opposite ends of $M \times I$ (see Section 4.3 below). By running this homotopy partially forward, then waiting, and then backward, in increasing increments, one can construct a homotopy (rel boundary) from $L^{\prime} \times I \subset M \times I$ to $A=A^{\prime}+(L \times I)-A^{\prime} \rightarrow M \times I$, where we write the composition of homotopies additively from left to right, and $-A^{\prime}$ is the oppositely oriented $A^{\prime}$ which runs from $L$ back to $L^{\prime}$ (see Figure 13). Since $A^{\prime}$ and $-A^{\prime}$ each support an order 1 Whitney tower, $A$ supports an order 1 Whitney tower such that no Whitney disks intersect the part of $M \times I$ containing $L \times I$. (If $\tau_{0}\left(L^{\prime}\right) \neq \tau_{0}(L)$ then this construction would still yield $A$ admitting an order 1 Whitney tower, but there 

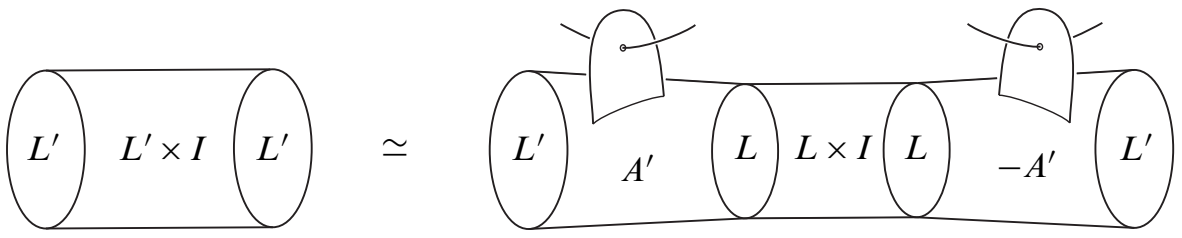

Figure 13: If $L^{\prime}$ is homotopic to $L$ by $A^{\prime}$, then $L^{\prime} \times I \subset M \times I$ is homotopic to $A^{\prime}+(L \times I)-A^{\prime}$ ↔ $M \times I$.

would necessarily be canceling pairs of intersections with one point from each pair in $A^{\prime}$ and the other in $-A^{\prime}$, so that the corresponding Whitney disks would have to intersect the part of $M \times I$ containing $L \times I$.) Now $S$ is homotopic into the part of $M \times I$ containing $L \times I$, where Whitney disks can be found for all intersections between $S$ and $L \times I$ such that the Whitney disks do not intersect the parts of $M \times I$ containing $A^{\prime}$ and $-A^{\prime}$ (since $\left.\tau_{0}(L \times I, S)=0\right)$. It follows from the homotopy invariance of $\tau_{1}$ that $\tau_{1}\left(L^{\prime}, S\right)=\tau_{1}(L, S)$, proving the claim.

So to prove the lemma we are free to use any link $L$ with $\tau_{0}(L)=z$. This will not be hard using a clasp link (Figure 3 and Section 4.4 below) whose intersections with $S$ can be paired by Whitney disks that are contained in neighborhoods of the arcs guiding the clasps.

Consider the case where $S$ is a separating sphere giving a connected sum decomposition $M=M^{\prime} \sharp_{S} M^{\prime \prime}$, and $z=+(g)_{i j}$, is represented by a single positively signed order zero tree (edge) oriented from $i$ to $j$, and labeled by $g \in \pi_{1} M$. Writing $g=h_{0} \prod_{l=1}^{n} g_{l} h_{l}$, where the $g_{l}$ are carried by $M^{\prime}$ and the $h_{l}$ are carried by $M^{\prime \prime}$, we can create $L$, with $\tau_{0}(L)=(g)_{i j}$, from the unlink by introducing a clasp guided by an arc from the $i-$ th to the $j$-th unlink component, which intersects $S$ in $2 n$ points, so that $A_{i}=L_{i} \times I$ has $2 n$ canceling pairs of intersections with $S$ in some interior slice of $M \times I$, as illustrated in Figure 14 for the case $n=2$.

As can be seen in Figure 14, these canceling pairs admit embedded Whitney disks, each of which winds along the band of the clasp and has a single interior intersection with $A_{j}=L_{j} \times I$. Using the product structure of $M \times I$, these Whitney disks can be made pairwise disjoint by a small perturbation. With our usual convention of orienting all edges towards the trivalent vertex, the group element labels on the trees associated to these Whitney disks can be seen to have the following pattern: If the whiskers on the Whitney disks are chosen to respect the factorization of $g$, then as we move through the Whitney disks from $A_{i}$ towards $A_{j}$ (right to left in the figure), the group elements labelling the $i$-edge read off increasing subwords of $g$, while the group elements labelling the $j$-edge read off decreasing subwords of $g^{-1}$, as 


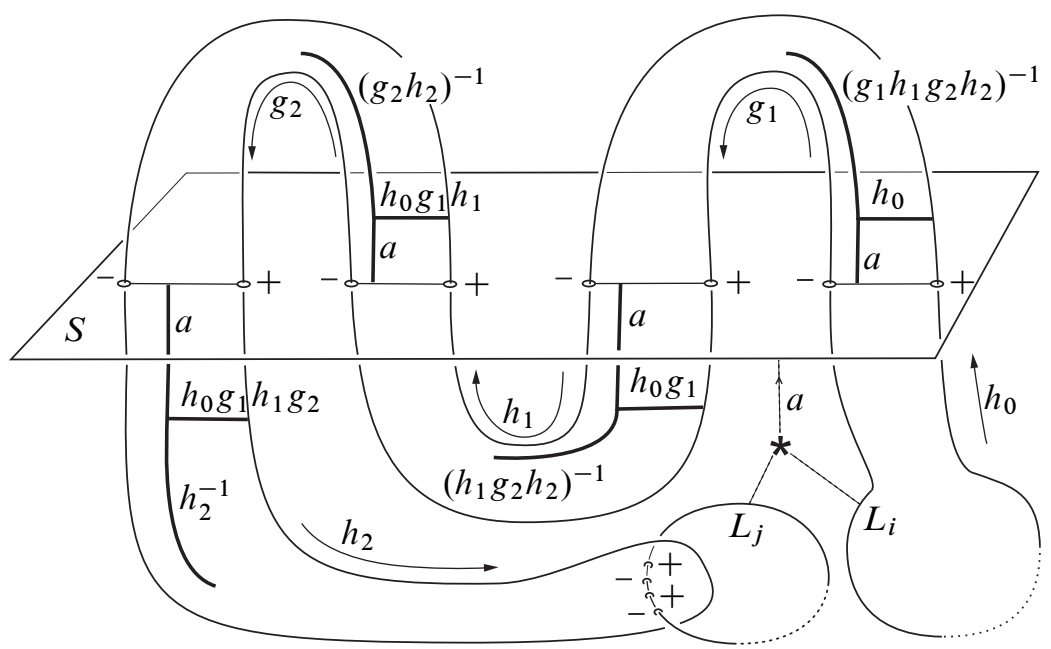

Figure 14: Four parallel Whitney disks pair intersections between a separating sphere $S$ and a clasp link $L$ with $\tau_{0}(L)=(g)_{i j}$ for $g=h_{0} g_{1} h_{1} g_{2} h_{2}$. The indicated decorations for the associated trees can be computed from the diagram, giving $\tau_{1}(L, a S)=\left(h_{0},\left(g_{1} h_{1} g_{2} h_{2}\right)^{-1}, a\right)_{i j S}-\left(h_{0} g_{1},\left(h_{1} g_{2} h_{2}\right)^{-1}, a\right)_{i j S}+$ $\left(h_{0} g_{1} h_{1},\left(g_{2} h_{2}\right)^{-1}, a\right)_{i j S}-\left(h_{0} g_{1} h_{1} g_{2}, h_{2}^{-1}, a\right)_{i j S}=\left(g, 1, a g_{1} h_{1} g_{2} h_{2}-\right.$ $\left.a h_{1} g_{2} h_{2}+a g_{2} h_{2}-a h_{2}\right)_{i j S}$, where the second equality comes from the HOL relations.

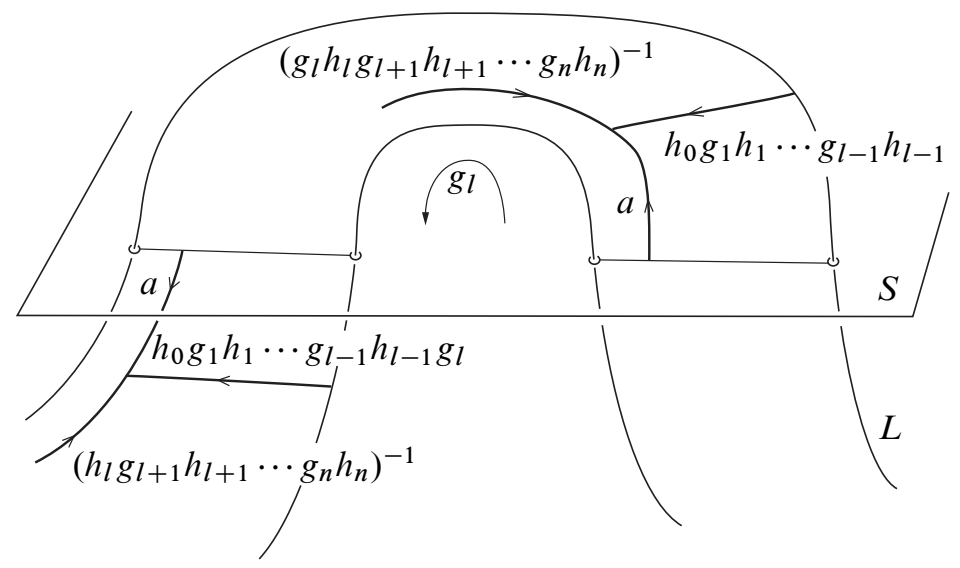

Figure 15

illustrated in Figure 15. A choice of whisker on $S$ determines an element $a \in \pi_{1} M$ labelling all the edges corresponding to $S$. Now using the right multiplication of the HOL relations (geometrically, changing the whiskers on the Whitney disks) to get the 
group element $g$ on the $i$-edge of each tree, simultaneously changes each $j$-edge label to 1 , and changes the $S$-edges exactly as described by the map $\sigma_{S}(g$ ) (see the example in Section 3.2.1 above), once the signs are taken into account, as can be checked in Figure 14 using our positive corner convention. Thus in this case, we have $\tau_{1}(L, a S)=\left(g, 1, a \sigma_{S}(g)\right)_{i j S}=\left(g, 1, \sigma_{a S}(g)\right)_{i j S}$ as desired.

It is easy to see that $\tau_{1}(L, S)$ behaves linearly under adding parallel copies of $S$, with each new copy of $S$ having $2 n$ more parallel Whitney disks, with appropriate sign changes for orientation-reversed copies of $S$.

Fixing $L$ and $z=(g)_{i j}$, and taking a different (nonhomotopic) separating sphere $S^{\prime}$, gives a different factorization of $g$, but the same construction works simultaneously to confirm the computation for $\pi_{1} M$-linear combinations of separating spheres:

$$
\tau_{1}\left(L, a S+a^{\prime} S^{\prime}\right)=\left(g, 1, a \sigma_{S}(g)\right)_{i j S}+\left(g, 1, a^{\prime} \sigma_{S}^{\prime}(g)\right)_{i j S^{\prime}}=\left(g, 1, \sigma_{a S+a^{\prime} S^{\prime}}(g)\right) .
$$

And for $z=-(g)_{i j}$, changing the sign of the clasp gives a global change of sign for $\tau_{1}(L, S)$.

Since this entire construction takes place in a neighborhood of the guiding arc for the clasp determining $g$, it can be iterated any number of times giving the result for $z=\sum_{i \leq j} \sum_{p} e_{p} \cdot\left(g_{p}\right)_{i j}$ and combinations of separating spheres.

Checking the case where $S$ is nonseparating follows the same construction: Noting that the construction only depends on the arc guiding the clasp, Figure 16 shows a more schematic illustration of the computation of $\tau_{1}(z, S)$ for $S$ a cross-section of an $S^{1} \times S^{2}$ factor of $M$, in the case $z=+(g)_{i j}$ with $g=h_{0} x^{3} h_{1} x^{-3} h_{2}$, where $x$ represents a generator of the circle factor in $\pi_{1} M$. Collecting terms and using HOL relations again gives $\tau_{1}(L, a S)=\left(g, 1, \sigma_{a S}(g)\right)_{i j S}$ (check with the example in Section 3.2.2 above).

\subsection{The indeterminacy subgroup $\Phi(z)$}

In the definition given in Section 1.3 of the relative order 1 intersection tree $\tau_{1}\left(L_{z}, L\right):=$ $\tau_{1}(A)$, there is an obvious indeterminacy coming from changing the components of $A$ by connected sums with 2-spheres. By Lemma 3.2.1 this indeterminacy is characterized by the subgroup $\Phi(z)$ which we define to be the span of the elements

$$
\sum_{i \leq j} \sum_{p} e_{p} \cdot\left(g_{p}, 1, a \sigma_{S}\left(g_{p}\right)\right)_{i j k} \in \mathcal{T}_{1}\left(\pi_{1} M\right) / \operatorname{INT}(z)
$$

for $z=\sum_{i \leq j} \sum_{p} e_{p} \cdot\left(g_{p}\right)_{i j}$ written in normal form, where $a$ ranges over $\pi_{1} M$ and $S$ ranges over a generating set for $\pi_{2} M$. Each such element corresponds to the effect on $\tau_{1}(A)$ of tubing a $2-$ sphere $a S$ into the $k$-th component $A_{k}$. 


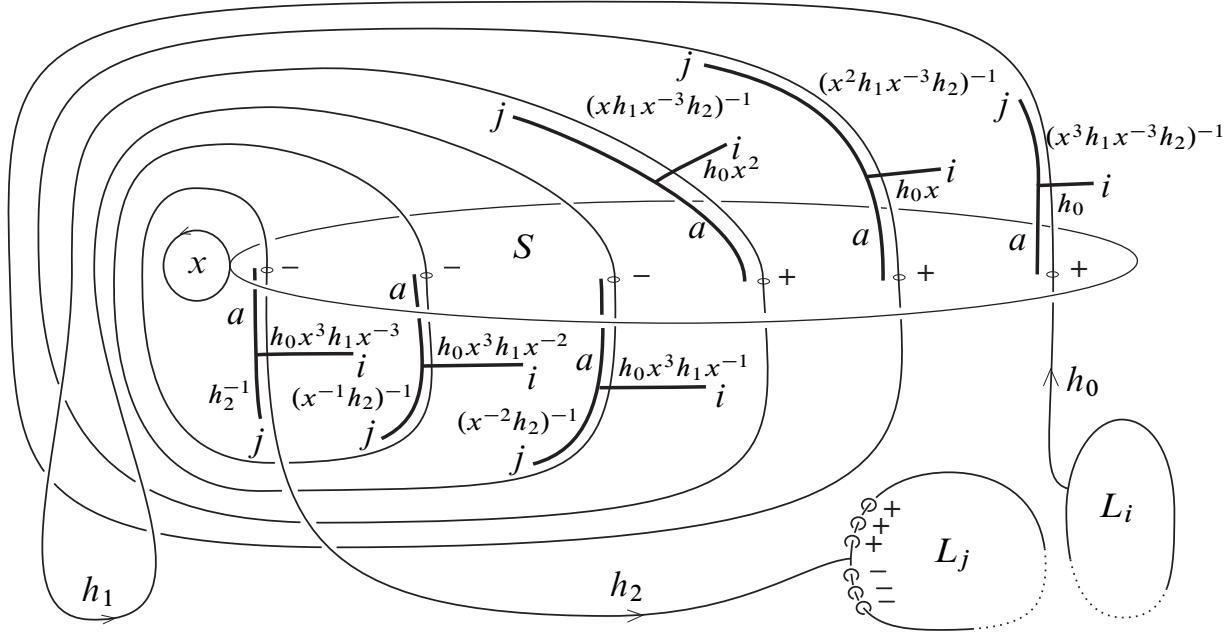

Figure 16: The arc from $L_{i}$ to $L_{j}$ representing $g=h_{0} x^{3} h_{1} x^{-3} h_{2}$ guides a clasp which, via the construction of Figure 14, leads to six Whitney disks - one for each intersection between the arc and the nonseparating sphere $S$. Only the trees of the Whitney disks are shown, with edges decorated by group elements as computed from the diagram.

\section{Proof of Theorem 3}

This section clarifies the definition of the relative order 1 intersection tree $\tau_{1}\left(L_{z}, L\right)$ for links of null-homotopic knots given in Section 1.3, and introduces notions relevant to the proof of Theorem 3. The proof is based on three lemmas which are left to the end of the section.

We remark that for links in 3-manifolds the equivalence relations of singular concordance (cobounding immersed annuli in $M \times I$ ) and homotopy (level-preserving singular concordance) coincide by $[14 ; 15]$.

\subsection{The $\Psi$ whisker-change actions}

The invariants $\tau_{0}(A)$ and $\tau_{1}(A)$ depend on fixing whiskers on the $m$ components of $A$. In the present setting, the corresponding indeterminacies are characterized by the following (left) actions of the $m$-fold cartesian product $\Psi:=\left(\pi_{1} M\right)^{m}$ on $\mathcal{T}_{0}\left(\pi_{1} M\right)$ and $\mathcal{T}_{1}\left(\pi_{1} M\right)$ :

The action of $\psi=\left(\psi_{1}, \psi_{2}, \ldots, \psi_{m}\right) \in \Psi$ on $\mathcal{T}_{0}\left(\pi_{1} M\right)$ and $\mathcal{T}_{1}\left(\pi_{1} M\right)$, respectively, is defined on generators by

$$
\left(g_{p}\right)_{i j} \mapsto\left(\psi_{i} g_{p} \psi_{j}^{-1}\right)_{i j} \quad \text { and } \quad(a, b, c)_{i j k} \mapsto\left(\psi_{i} a, \psi_{j} b, \psi_{k} b\right)_{i j k}
$$




\subsection{Latitudes of singular concordances}

Let $K \subset M$ be a knot, and let $A \rightarrow M \times I$ be a singular concordance from $K$ to $K^{\prime}$. A path that goes along a whisker on $K$ in $M \times\{0\}$, then along $A$ to $K^{\prime}$, and then along a whisker on $K^{\prime}$ in $M \times\{1\}$ is called a latitude of $A$. Via projection to $M \times\{0\}$, a latitude of $A$ determines an element in $\pi_{1} M$.

For a singular concordance $A$ of a link $L \subset M$, latitudes of the components determine an element $\psi \in \Psi$.

We write compositions of singular concordances additively from left to right. Note that if $A_{\psi}$ and $A_{\phi}$ have latitudes determining $\psi$ and $\phi$ respectively, then $A_{\psi}+A_{\phi}$ has latitudes determining the product $\psi \phi$. Also, if $-A_{\psi}$ denotes the inversion of $A_{\psi}$, then $-A_{\psi}$ has latitudes determining $\psi^{-1}$. Basepoints are assumed to be taken in $M \times\{0\}$ (unless otherwise stated), so for $n=0,1$ we have

$$
\tau_{n}\left(-A_{\psi}\right)=-\psi^{-1} \cdot \tau_{n}\left(A_{\psi}\right),
$$

as well as

$$
\begin{aligned}
& \tau_{n}\left(A_{\psi}+A_{\phi}\right)=\tau_{n}\left(A_{\psi}\right)+\psi \cdot \tau_{n}\left(A_{\phi}\right) \\
& \tau_{n}\left(A_{\psi}-A_{\phi}\right)=\tau_{n}\left(A_{\psi}\right)-\psi \phi^{-1} \cdot \tau_{n}\left(A_{\phi}\right)
\end{aligned}
$$

and

where the actions of $\Psi$ on $\mathcal{T}_{0}$ and $\mathcal{T}_{1}$ are both denoted by ". ". Note that when inverting a singular concordance, the orientation of $M \times I$ is assumed to remain fixed. Specific orientation conventions are suppressed from notation, but consistent throughout the examples.

\subsection{Order 1 Whitney concordance}

Consider $L$ and $L^{\prime}$ with $\tau_{0}(L)=z=\tau_{0}\left(L^{\prime}\right) \in \mathcal{T}_{0}\left(\pi_{1} M\right)$, for some choices of whiskers. Placing $L$ and $L^{\prime}$ in either end of $M \times I$ and connecting the components of singular null-concordances $D$ and $D^{\prime}$ by disjointly embedded tubes gives a singular concordance $A$ from $L$ to $L^{\prime}$. Using the whiskers on $L \subset M \times\{0\}$, we have

$$
\tau_{0}(A)=\tau_{0}(D)-\psi \cdot \tau_{0}\left(D^{\prime}\right)=z-\psi \cdot z
$$

for some $\psi \in \Psi$ which depends on the whiskers and the choices of tubes. Since we can rechoose the tubes in the construction of $A$ to realize any element in $\Psi$, we can arrange for $\psi$ to be trivial, so that $\tau_{0}(A)=0 \in \mathcal{T}_{0}\left(\pi_{1} M\right)$, which implies that $L$ and $L^{\prime}$ are order 1 Whitney concordant.

(Note that $A$ can be constructed to be the trace of a homotopy from $L$ to $L^{\prime}$ : Take $D$ and $D^{\prime}$ to be homotopies to the unlink, and realize the tubes by isotopies of the unlink components.) 
It follows that $\tau_{0}(L) \in \mathcal{T}_{0}\left(\pi_{1} M\right)$ classifies order 1 Whitney concordance for links of null-homotopic knots, since the converse is given by the last line of the proof of Theorem 2 in Section 2.6.

Note that the same argument shows that $\tau_{1}(L) \in \mathcal{T}_{1}\left(\pi_{1} M\right)$ classifies order 2 Whitney concordance, hence stable concordance, for $L$ with $\tau_{0}(L)=0$. But the classification for $\tau_{0}(L) \neq 0$ - as per Theorem 3 - is more subtle (as is the classification in the case of essential knots given in Theorem 5 of Section 6).

\subsection{Clasp links}

For each $z \in \mathcal{T}_{0}\left(\pi_{1} M\right)$ the link $L_{z}$ in Theorem 3 can be taken to be a clasp link constructed from an $m$-component unlink $U$ in the following way. Fix whiskers and orientations on $U$. For each $e_{p} \cdot\left(g_{p}\right)_{i j}$ in $z$, choose $\left|e_{p}\right|$ embedded guiding arcs joining $U_{i}$ and $U_{j}$ which represent $g_{p} \in \pi_{1} M$. Replace each guiding arc by adding a band to the $i$-th component which "clasps" the $j$-th component as illustrated in Figure 3, with the sign of the clasp taken so that the intersection point in the trace of the homotopy which undoes the clasp has sign equal to the sign of $e_{p} \in \mathbb{Z}$.

For given $z$, any two clasp links $L_{z}$ and $L_{z}^{\prime}$ are order 1 Whitney equivalent, since $\tau_{0}\left(L_{z}\right)=z=\tau_{0}\left(L_{z}^{\prime}\right)$. Fixing a chosen clasp link $L_{z}$ gives the classification of Theorem 3. Up to isotopy, there are three kinds of indeterminacies coming from the choices in the construction of a clasp link: twisting of the clasps (around the guiding arcs), knotting and linking of the guiding arcs, and the configurations of the guiding arc endpoints on $U$. As illustrated in Section 5 below, for any two choices the difference $\tau_{1}\left(L_{z}, L_{z}^{\prime}\right)$ can be explicitly computed.

\subsection{Stabilizers of the $\Psi$-action on $\mathcal{T}_{0}$}

The proof of Theorem 3, as well as its constraints, will depend on understanding the stabilizers of the $\Psi$-action on $\mathcal{T}_{0}\left(\pi_{1} M\right)$.

For $z \in \mathcal{T}_{0}\left(\pi_{1} M\right)$ written in normal form (as in Section 3.1), the action $z \mapsto \psi \cdot z$ of $\psi=\left(\psi_{1}, \psi_{2}, \ldots, \psi_{m}\right) \in \Psi$ is

$$
z=\sum_{i \leq j} \sum_{p} e_{p} \cdot\left(g_{p}\right)_{i j} \mapsto \sum_{i \leq j} \sum_{p} e_{p} \cdot\left(\psi_{i} g_{p} \psi_{j}^{-1}\right)_{i j},
$$

so $\psi$ is in the stabilizer $\Psi_{z}$ of $z$ if and only if for all $i$ and $j$

$$
\sum_{p} e_{p} \cdot\left(g_{p}\right)_{i j}=\sum_{p} e_{p} \cdot\left(\psi_{i} g_{p} \psi_{j}^{-1}\right)_{i j},
$$


which means that each pair of factors $\psi_{i}$ and $\psi_{j}$ permutes the elements $g_{p}$, and if $i=j$ possibly sends some $g_{p}$ to $g_{p}^{-1}$. (Of course permuting the $g_{p}$ in $z_{i j}$ is not sufficient for $\psi$ to be in $\Psi_{z}$ since the coefficients of the permuted elements would also have be equal.)

A stabilizer $\Psi_{z}$ is called untwisted if for all $\psi \in \Psi_{z}$, and all $i$ and $j$,

$$
g_{p}=\psi_{i} g_{p} \psi_{j}^{-1}
$$

and $\Psi_{z}$ is called twisted otherwise. So untwisted stabilizers are exactly those which induce the identity map on the fundamental group elements in $z_{i j}$ for all $i$ and $j$.

Will also refer to an element $\psi$ of $\Psi_{z}$ as twisted or untwisted, depending on whether $\psi$ induces a nontrivial or trivial permutation of the elements in $z$; so that $\Psi_{z}$ is untwisted if every $\psi$ in $\Psi_{z}$ is untwisted. As discussed in Section 5.2 below, in a sense "most" $z$ have untwisted stabilizers.

\section{6 $\tau_{1}\left(L_{z}, L\right)$ is well-defined}

As discussed in Section 4.3 above, it is natural to interpret the values of the "absolute" invariants $\tau_{0}(L)$ and $\tau_{1}(L)$ modulo the respective $\Psi$-actions. This means that these invariants are completely independent of choices of whiskers.

In the definition of $\tau_{1}\left(L_{z}, L\right)$ we consider $L_{z}$ to be fixed, including the choice of whiskers. Then, for each $L$ the value of $\tau_{1}\left(L_{z}, L\right)$ in the quotient of $\mathcal{T}_{1}\left(\pi_{1} M\right)$ by $\operatorname{INT}(z)$ and $\Phi(z)$ is taken modulo the action of the stabilizer $\Psi_{z}$. The reason for this is essentially due to Lemma 4.8 .2 below as will become clear shortly.

Let $A$ and $A^{\prime}$ be singular concordances from $L_{z}$ to $L$ supporting order 1 Whitney towers. Then the composition $A-A^{\prime}$ is a singular self-concordance of $L_{z}$ supporting an order 1 Whitney tower. By Lemma 4.8.2 below, latitudes of $A-A^{\prime}$ determine an element $\psi \in \Psi_{z}$. Since $\Psi_{z}$ is assumed to be untwisted, by Lemma 4.8.3 below there exists a self-homotopy $H_{\psi^{-1}}^{0}$ of $L_{z}$ with latitudes determining $\psi^{-1}$ such that $\tau_{1}\left(H_{\psi^{-1}}^{0}\right)=0 \in \mathcal{T}_{1}\left(\pi_{1} M\right)$. Now the singular self-concordance $A-A^{\prime}+H_{\psi^{-1}}^{0}$ of $L_{z}$ has latitudes determining the trivial element $\psi \psi^{-1} \in \Psi$, so by Lemma 4.8.1 below, $A-A^{\prime}+H_{\psi^{-1}}^{0}$ is regularly homotopic (rel $\partial$ ) to the connected sum of $L_{z} \times I$ with disjointly embedded 2-spheres. By the homotopy invariance of $\tau_{1}$ and the definition of $\Phi(z)$ given in Section 3.3 we have

$$
\tau_{1}\left(A-A^{\prime}+H_{\psi^{-1}}^{0}\right)=\tau_{1}(A)-\psi \cdot \tau_{1}\left(A^{\prime}\right) \in \Phi(z),
$$

so $\tau_{1}\left(L_{z}, L\right)$ does not depend on the choice of $A$. 


\subsection{The equivalence of statements (i)-(iv) in Theorem 3}

We will show the equivalence of (i) and (iii); the equivalence of (ii), (iii) and (iv) follows from Theorem 4 in Section 3.

If $L$ and $L^{\prime}$ are order 2 Whitney concordant, then they cobound an immersed annulus $A^{\prime}$ which supports an order 2 Whitney tower. So any singular concordance $A$ used to compute $\tau_{1}\left(L_{z}, L\right)$ can be extended by $A^{\prime}$ to compute $\tau_{1}\left(L_{z}, L^{\prime}\right)=\tau_{1}\left(A+A^{\prime}\right)=$ $\tau_{1}\left(L_{z}, L\right)$, since $\tau_{1}\left(A^{\prime}\right)$ vanishes.

On the other hand, the equality $\tau_{1}\left(L_{z}, L^{\prime}\right)=\tau_{1}\left(L_{z}, L\right)$ means that if $A$ and $A^{\prime}$ are order 1 Whitney concordances from $L_{z}$ to $L$ and $L^{\prime}$ respectively, then $\tau_{1}\left(A^{\prime}\right)=$ $\psi \cdot \tau_{1}(A)$ modulo $\Phi(z)$, for some $\psi \in \Psi_{z}$. After realizing $\operatorname{INT}(z)$ relations and taking connected sums with some 2-spheres (if necessary) we may arrange that $\tau_{1}\left(A^{\prime}\right)=$ $\psi \cdot \tau_{1}(A) \in \mathcal{T}_{1}\left(\pi_{1} M\right)$. By Lemma 4.8.3, there exists a self-homotopy $H_{\psi^{-1}}^{0}$ of $L_{z}$ with $\tau_{1}\left(H_{\psi^{-1}}^{0}\right)=0$ and latitudes determining $\psi^{-1}$. Now the composition $-A+H_{\psi^{-1}}^{0}+A^{\prime}$ is an order 1 Whitney concordance from $L$ to $L^{\prime}$, and taking the whiskers on $L_{z}$ at the end of $-A$ we have

$$
\tau_{1}\left(-A+H_{\psi^{-1}}^{0}+A^{\prime}\right)=-\tau_{1}(A)+\psi^{-1} \cdot \tau_{1}\left(A^{\prime}\right)=-\tau_{1}(A)+\psi^{-1} \psi \cdot \tau_{1}(A)=0
$$

so by Theorem 4 it follows that $-A+H_{\psi^{-1}}^{0}+A^{\prime}$ is homotopic to a singular concordance from $L$ to $L^{\prime}$ which supports an order 2 Whitney tower.

\subsection{Lemmas}

The proof of Theorem 3 is completed by the following three lemmas.

Lemma 4.8.1 [28] If a singular self-concordance $A$ of a knot $K \subset M$ has a latitude that represents the trivial element $1 \in \pi_{1} M$, then $A$ is homotopic (rel $\partial$ ) to the connected sum of an embedded 2-sphere and the embedded annulus $K \times I$ in $M \times I$.

Furthermore, if $A$ also supports a Whitney tower of order 1 (or greater), and $K$ has trivial order zero intersections with 2-spheres in $M$, then the above homotopy (rel $\partial$ ) can be taken to be a regular homotopy.

Note that $K$ is not assumed to be null-homotopic in Lemma 4.8.1.

Proof The properly immersed $A$ projects to a map of a torus $T \rightarrow M=M \times\{0\}$, with the image of a latitude in $A$ projecting to a loop $C$ in the image of $T$ which is contractible in $M$. The inverse image $\bar{C}$ of $C$ is algebraically dual to the inverse image $\bar{K}$ of $K$ in the domain torus $T$, so after a homotopy we may assume that $\bar{C}$ is 
geometrically dual to $\bar{K}$ and embedded. Then $C$ bounds an immersed 2-disk $D$ in $M$ which surgers the map $T \rightarrow M$ to a map of a 2-sphere $S \rightarrow M$. This surgery lifts to a homotopy (rel $\partial$ ) of $A$ in $M \times I$ to $K \times I \sharp S^{\prime}$, where $S^{\prime}$ is a lift of $S$. By Lemma 2.6.1, $S^{\prime}$ can be assumed to be embedded.

The second statement of Lemma 4.8.1 follows from the fact that, up to isotopy, a homotopy of surfaces in a 4-manifold consists of a sequence of finger moves, Whitney moves, and cusp homotopies. If the cusp homotopies occur in canceling pairs, then they can be replaced (up to isotopy) by finger moves (pairs of births) and Whitney moves (pairs of deaths), so that the homotopy is a regular homotopy. So, if $K \times I \sharp S^{\prime}$ and $A$ both have all self-intersections occurring in canceling pairs - as will be the case under the further assumptions - then the above homotopy may be arranged to be a regular homotopy.

Lemma 4.8.2 For any $z \in \mathcal{T}_{0}\left(\pi_{1} M\right)$, let $L_{z} \subset M$ be any link such that $\tau_{0}\left(L_{z}\right)=$ $z \in \mathcal{T}_{0}\left(\pi_{1} M\right)$, and let $A_{\psi} \rightarrow M \times I$ be any singular self-concordance of $L_{z}$, with latitudes determining $\psi \in \Psi$. Then:

If $A_{\psi}$ admits an order one Whitney tower, then $\psi \in \Psi_{z}$.

Proof As described in Section 4.3, there exists a self-homotopy $H_{\psi}$ of $L_{z}$ with latitudes determining $\psi$ such that $\tau_{0}\left(H_{\psi}\right)=z-\psi \cdot z \in \mathcal{T}_{0}\left(\pi_{1} M\right)$. Since the composition $A_{\psi}-H_{\psi}$ has latitudes representing the trivial element $\psi \psi^{-1} \in \Psi$, it follows from Lemma 4.8.1 that $A_{\psi}-H_{\psi}$ is homotopic (rel $\partial$ ) to the connected sum of $L_{z} \times I$ with some 2-spheres. By the homotopy invariance of $\tau_{0}$, and since null-homotopic knots have trivial order zero intersections with 2-spheres, we have that $\tau_{0}\left(A_{\psi}-H_{\psi}\right)=0 \in$ $\mathcal{T}_{0}\left(\pi_{1} M\right)$. So, computing in $\mathcal{T}_{0}\left(\pi_{1} M\right)$ we have

$$
0=\tau_{0}\left(A_{\psi}-H_{\psi}\right)=\tau_{0}\left(A_{\psi}\right)-\tau_{0}\left(H_{\psi}\right)=0-(z-\psi \cdot z)
$$

since $\tau_{0}\left(A_{\psi}\right)=0$, which shows that $\psi \in \Psi_{z}$.

Lemma 4.8.3 Let $L_{z} \subset M$ be a clasp link with $\tau_{0}\left(L_{z}\right)=z \in \mathcal{T}_{0}\left(\pi_{1} M\right)$, such that $\Psi_{z}$ is untwisted. Then for any $\psi \in \Psi_{z}$, there exists a self-homotopy $H_{\psi}^{0}$ of $L_{z}$ with latitudes determining $\psi$, and an order one Whitney tower $\mathcal{W}$ on $H_{\psi}^{0}$, such that $\tau_{1}(\mathcal{W})=0 \in \mathcal{T}_{1}\left(\pi_{1} M\right)$.

Proof The construction of $H_{\psi}^{0}$ has three steps, and is based on the observation that undoing and redoing clasps describes a self-homotopy supporting an order 1 Whitney tower whose Whitney disks are described by the guiding arcs of the clasps (Figure 17): 

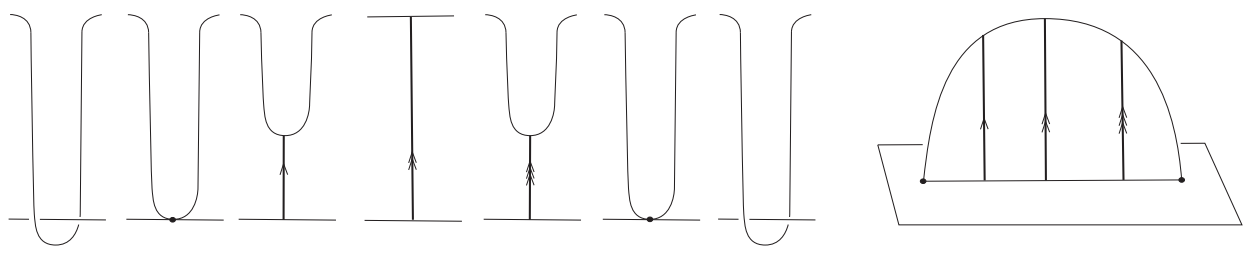

Figure 17: The guiding arc traces out a Whitney disk as a clasp is undone then redone.

First, undoing the clasps by crossing changes describes a homotopy from $L_{z}$ to the unlink $U$, with the guiding arcs tracing out corners of embedded Whitney disks near the crossing-change intersection points (Figure 3). The components of $U$ can be assumed to lie in small 3-balls, and the guiding arcs between $U_{i}$ and $U_{j}$ represent the elements $\left(g_{p}\right)_{i j}$ in $z_{i j}$.

Now, the untwistedness of $\psi$ means that $g_{p}=\psi_{i} g_{p} \psi_{j}^{-1} \in \pi_{1} M$ for all $i$ and $j$ (and $p$ which depends on $i$ and $j$ ) which is exactly the condition that a self-isotopy of $U$ around loops representing $\psi$ extends to self-homotopies of the guiding arcs and whiskers which are attached to $U$. Each of these self-homotopies of guiding arcs traces out the center rectangle of an immersed Whitney disk in $\mathcal{W}$. The self-isotopy of $U$ extends to a self-isotopy of the small 3-ball neighborhoods of the components of $U$ together with a neighborhood of the whiskers, which forms a thickened wedge of arcs. So the only singularities created in this second step of the construction are possible crossing changes between the guiding arcs which are interior intersections among the Whitney disks of $\mathcal{W}$.
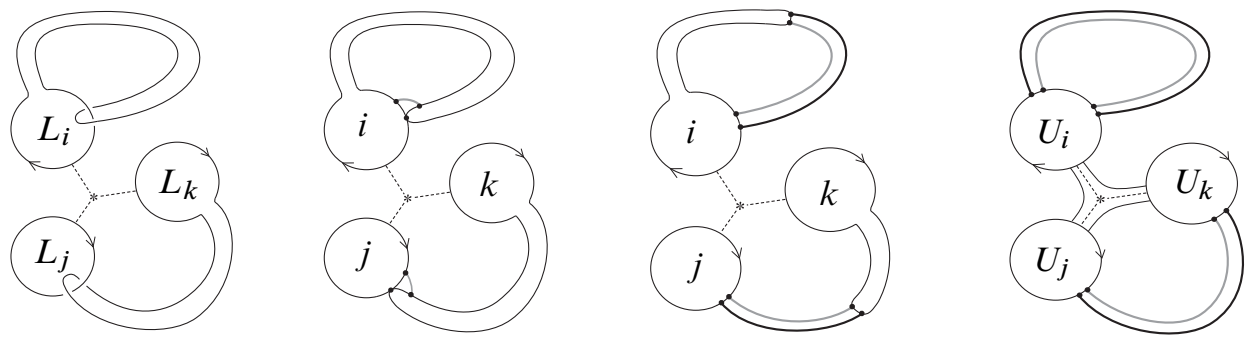

Figure 18: From left to right, the first part of the homotopy $H_{\psi}^{0}$, and from right to left the third part. The guiding arcs trace out Whitney disks, and gray arcs trace out an extension of the Whitney section. The right most picture includes the surface $F$.

The third step is to run the first homotopy backwards from $U$ to $L_{z}$, recreating the clasps by crossing changes, each of which gives an intersection point which forms a canceling pair with the corresponding intersection created when undoing the clasps 
in the first step. In this third step the guiding arcs shrink down and disappear at the intersection points, tracing out the remaining corners of the Whitney disks in $\mathcal{W}$.

The union $H_{\psi}^{0}$ of these three steps is a self-homotopy of $L_{z}$ which by construction has latitudes determining $\psi$. Assuming for the moment that the Whitney disks are framed, it is clear that $\tau_{1}(\mathcal{W})=0 \in \mathcal{T}_{1}\left(\pi_{1} M\right)$ because the only singularities are interior intersections among the Whitney disks which do not contribute to $\tau_{1}$ (they are order 2 intersections). It remains to show that the Whitney disks can be arranged to be framed, which will follow essentially from the fact that Chernov's affine self-linking numbers for framed knots in 3-manifolds vanish modulo 2 [4].

Recall that the normal section which defines the Whitney disk framing obstruction is defined by pushing one Whitney disk boundary arc tangentially along its surface sheet and pushing the other boundary arc in the normal direction to its surface sheet. Observe that after a small isotopy ( $\pi / 2$ radians in the normal circle bundle) the part of the section that was pushed normally to a surface sheet will lie in the surface sheet, except for a short arc near each intersection point (in the right-hand side of Figure 4 picture the back half of the dotted loop sliding up to the top of the torus). This isotopy does not change the framing obstruction, and will make it easier to compute the framing obstructions in the construction of $\mathcal{W}$.

Push-offs of the Whitney disks in $\mathcal{W}$ extending the Whitney sections are given by parallel copies of the guiding arcs in the above description of $H_{\psi}^{0}$ (Figure 18). As shown in Figure 18, the parts of the Whitney disks in the first and third homotopies are disjoint from their push-offs, and so the only possible contributions to framing obstructions can occur in the second part of the homotopy, which we will call $H$.

At the start of $H$ we have guiding arcs and their parallel copies running between components of $U$. The components of $U$ bound disjointly embedded 2-disks (whose interiors are disjoint from all guiding arcs), and the guiding arc pairs determine bands between these 2-disks. After thickening the whiskers on $U$ to a wedge of bands joined near the basepoint of $M$, the resulting embedded surface $F$ (shown in the right-most picture of Figure 18) gives a normal framing on each of the (embedded) loops representing the $\left(g_{p}\right)_{i j}$.

Now $H$ is a self-homotopy of the union of $U$ together with its whiskers and the guiding arcs. Clearly $H$ extends to a homotopy of $F$ (which lies in a neighborhood of $U$ union whiskers and guiding arcs). We may assume that $H$ restricts to a self-isotopy on the subdisk of $F$ which is the union of the disks bounded by $U$ and the wedge of bands containing the whiskers. At the end of $H$ the guiding arcs are back where they started, but the parallel copies might not be, since the twisting of the bands may have changed (by full twists) during $H$. The framing obstruction on each Whitney 


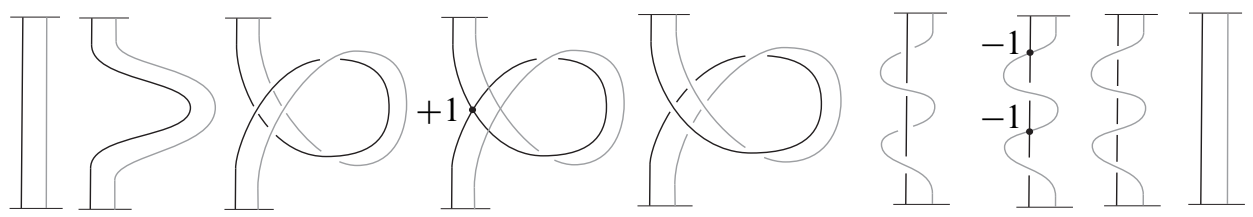

Figure 19: An interior twist on a Whitney disk is the local introduction of a self-intersection. Creation of a \pm 1 -intersection changes the framing obstruction by $\mp 2$, as can be seen in the intersections with a normal push-off of the Whitney disk shown in grey. Note that near the self-intersection of the Whitney disk there are a pair of intersections between the Whitney disk and its push-off which are just artifacts of the immersion of the disk bundle and do not contribute to the framing obstruction.

disk in $\mathcal{W}$ is equal to this change in framing of the corresponding loop in $F$ at the end of $H$. By the main results of [4], these relative framings can only differ by an even integer. So, after perhaps performing interior twists (which do not contribute to $\tau_{1}$ ) as illustrated in Figure 19, the Whitney disks in the above construction of $\mathcal{W}$ on $H_{\psi}^{0}$ can be assumed to be framed.

\section{Examples}

This section computes change-of-base-link formulas for $\tau_{1}\left(L_{z}, \cdot\right)$, characterizes twisted stabilizers, and shows how twisted $\Psi_{z}$ can lead to nontrivial indeterminacies in $\tau_{1}\left(L_{z}, \cdot\right)$.

\subsection{Clasp links}

For given $z \in \mathcal{T}_{0}\left(\pi_{1} M\right)$, a clasp link $L_{z}$ is determined up to isotopy by choices at three steps in the construction: the configuration of endpoints of guiding arcs on the unlink, the isotopy classes (rel endpoints) of the guiding arcs, and the (relative) framing of the band corresponding to the thickening of the guiding arc to a clasp. This subsection illustrates the computation of the order 1 relative intersection tree $\tau_{1}\left(L_{z}, L_{z}^{\prime}\right)$ for clasp links $L_{z}$ and $L_{z}^{\prime}$, giving a "change of base-link" formula for Theorem 3.

5.1.1 Twisting clasps Figure 20 illustrates the trace $A$ of a homotopy between clasp links $L_{z}$ and $L_{z}^{\prime}$ which differ by one twist of a clasp representing $g \in \pi_{1} M$ decorating $(g)_{j i}$ in $z$. The canceling pair of intersections of $A$ admit an embedded Whitney disk whose interior is disjoint from $A$, but which is not framed, as illustrated in Figure 21. Figure 22 shows how an application of the boundary twist procedure corrects the framing 
on the Whitney disk at the cost of creating an interior intersection between the Whitney disk and $A$, giving $\tau_{1}\left(L_{z}, L_{z}^{\prime}\right)=(1,1, g)_{i i j}$, which is 2 -torsion by the AS relations. In general, twisting such a band $n$ times would give $\tau_{1}\left(L_{z}, L_{z}^{\prime}\right)=n(1,1, g)_{i i j}$, which only depends on the parity of $n$.
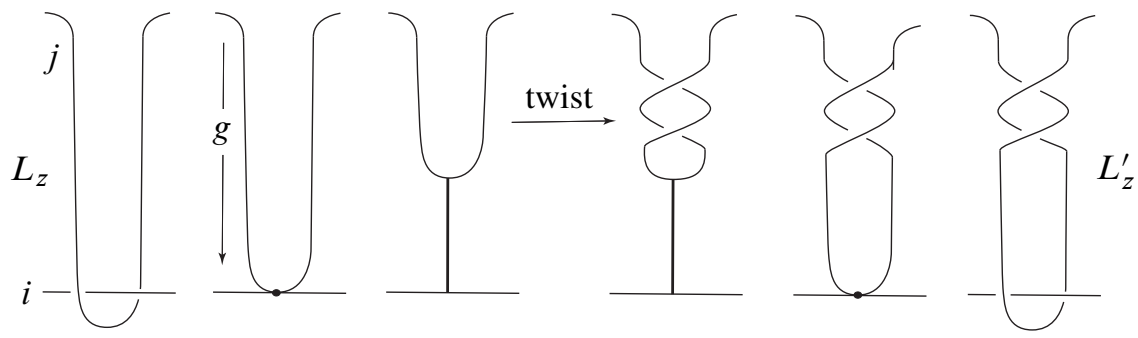

Figure 20: The trace of a homotopy between clasp links $L_{z}$ and $L_{z}^{\prime}$ which differ by a single twist in one clasp. The Whitney disk described by the vertical arcs is not framed, as shown in Figure 21.
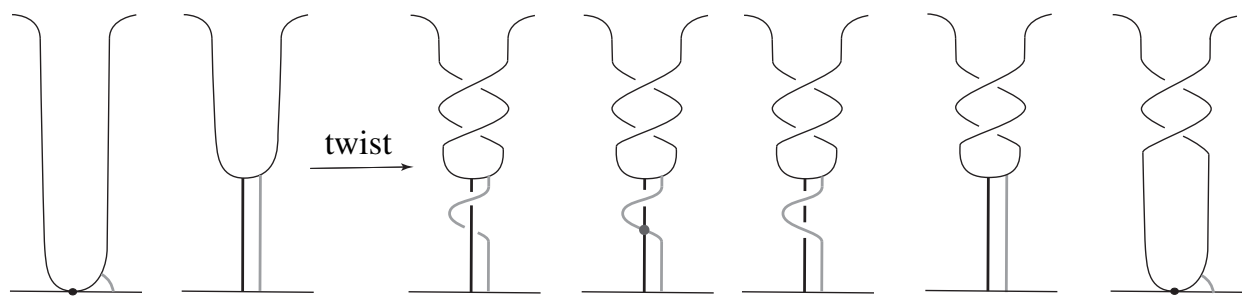

Figure 21: The grey arcs show an extension of the Whitney section over the interior of the Whitney disk. The single intersection between the extension and the Whitney disk indicates that the Whitney disk is not framed.

5.1.2 Choices of guiding arcs If $L_{z}$ and $L_{z}^{\prime}$ differ only by a homotopy (rel endpoints) of guiding arcs then $\tau_{1}\left(L_{z}, L_{z}^{\prime}\right)=0$ : This can be seen directly from the "band pass" move illustrated in Figure 23. Or, constructing a homotopy between $L_{z}$ and $L_{z}^{\prime}$ supporting Whitney disks described by the guiding arcs, the crossings between guiding arcs correspond to interior intersections between Whitney disks and do not contribute to $\tau_{1}$ (as in the proof of Lemma 4.8.3).

5.1.3 Guiding arc endpoint configurations Figure 24 shows the computation of the equality $\tau_{1}\left(L_{z}, L_{z}^{\prime}\right)=(1, g, h)_{i j k}$, where $L_{z}$ and $L_{z}^{\prime}$ are related by a single transposition of guiding arc endpoints along the $i$-th component. The reader familiar with finite type theory will recognize this as a decorated STU relation. 

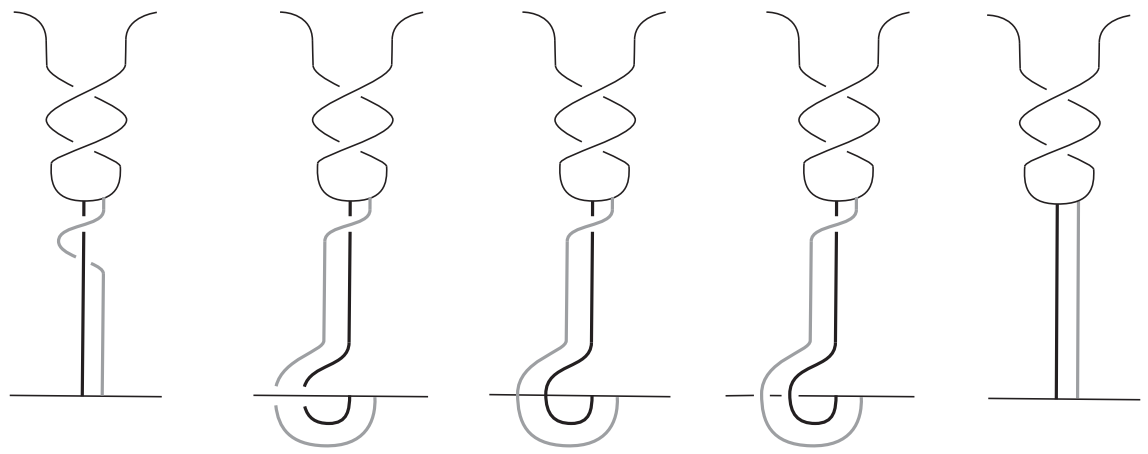

Figure 22: Changing the Whitney disk of Figures 20 and 21 by the indicated local procedure of introducing a boundary twist creates a framed Whitney disk at the cost of creating a single intersection (center picture) between the interior of the Whitney disk and the sheet that was twisted around.
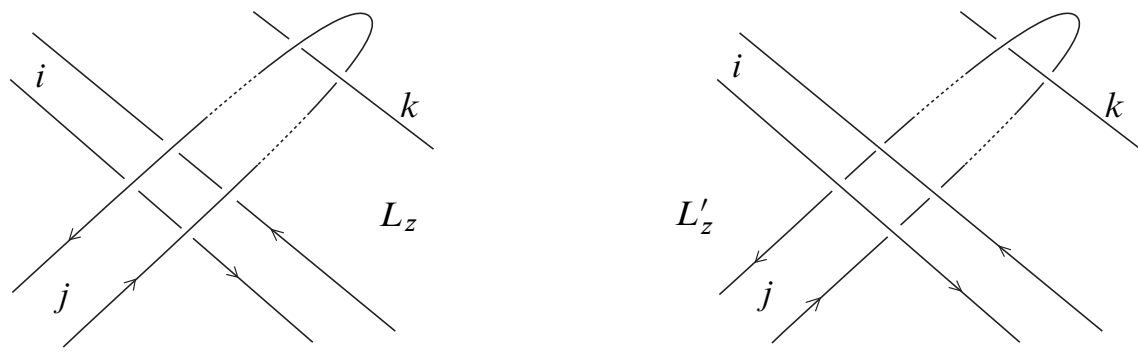

Figure 23: A homotopy which pushes a clasp of the $i$-th component across a clasp of the $j$-th component gives rise to two parallel oppositely oriented Whitney disks ("inside" the dotted $j$-clasp) whose interior intersections with the $k$-th component will have canceling trees.

The transposition of the two endpoints of the same guiding arc is illustrated in Figure 25, which computes the corresponding change in $\tau_{1}$ using the formula for intersections between Whitney disk boundaries. This computation will be used in Section 5.3 below.

\subsection{Twisted stabilizers}

This subsection points out that twisted stabilizers only occur in the presence of circle bundles over nonorientable surfaces with orientable total space, or Seifert fibered submanifolds containing singular fibers, and illustrates how twisted stabilizers can lead to self-homotopies of knots having nontrivial $\tau_{1}$.

To present the essential ideas with a minimum of subscripts we concentrate on the case where $z=\tau_{0}(K)$ for a knot $K \subset M$. Identifying $\mathcal{T}_{0}\left(\pi_{1} M\right)$ with $\mathbb{Z}\left[\pi_{1} M\right]$ modulo 

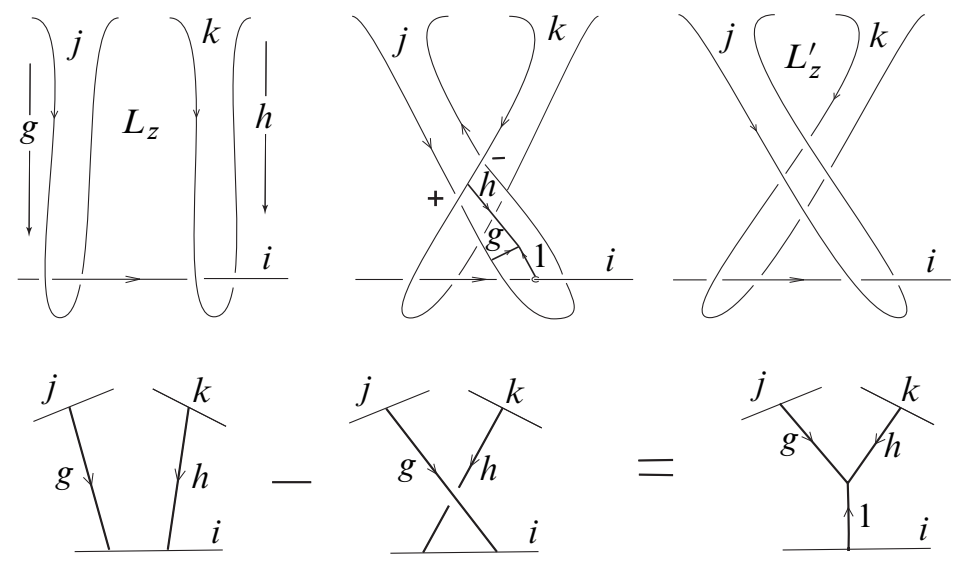

Figure 24: For clasp links $L_{z}$ and $L_{z}^{\prime}$ differing by the illustrated single transposition of guiding arc endpoints, $\tau_{1}\left(L_{z}, L_{z}^{\prime}\right)=(1, g, h)_{i j k}$.

inversion and trivial elements, and writing $z=\sum e_{i} g_{i}$ in normal form (with all $e_{i} \neq 0$, $g_{i} \neq 1$, and with $g_{i} \neq g_{j}^{ \pm 1}$ for $i \neq j$ ), any element $\psi$ in the stabilizer $\Psi_{z}$ of $z$ satisfies the equation

$$
\sum e_{i} \psi g_{i} \psi^{-1}=\sum e_{i} g_{i} \quad \text { modulo } g=g^{-1} .
$$

So the conjugation action of $\psi$ permutes the (finite) set $\left\{g_{i}^{ \pm 1}\right\}$, and by definition $\psi$ is twisted if this permutation is nontrivial, and untwisted if this permutation is the identity. Assuming that $\psi$ is twisted, there is a (least) natural number $2 \leq n \in \mathbb{N}$ such that $\psi^{n}$ induces the identity permutation on $\left\{g_{i}^{ \pm 1}\right\}$. Writing $\zeta(z):=\bigcap_{i} \zeta\left(g_{i}\right)$ for the intersection of the centralizers $\zeta\left(g_{i}\right)$ in $\pi_{1} M$ of the $g_{i}$, we have $\psi^{n} \in \zeta(z)$, but $\psi \notin \zeta(z)$. From work of Jaco and Shalen [18; 20; 19], Casson-Jungreis [3] and Gabai [11], noncyclic centralizers of nontrivial elements of $\pi_{1} M$ are carried by a codimension zero characteristic submanifold of $M$ which is Seifert fibered. Since $n$-th roots of any element are contained in the centralizer of that element, it can be checked that both $\zeta(z)$ and $\zeta\left(\psi^{n}\right)$ are carried by the same connected component $N$ of the characteristic submanifold of $M$.

It follows from Section 4 of [19] (in particular Theorem 4.4) that there are only two possible ways for $\psi^{n} \in \zeta(z)$, but $\psi \notin \zeta(z)$ : Either $\psi$ and $\left\{g_{i}\right\}$ are all carried by a submanifold $B \widetilde{\times} S^{1} \subset N$ which is the orientable circle bundle over the Möbius band, or $\psi$ is carried by a neighborhood of singular fiber in $N$ and $\left\{g_{i}\right\}$ lie in the canonical subgroup of $\pi_{1} N<\pi_{1} M$ consisting of elements represented by loops which project to orientation-preserving loops in a base surface for $N$. In both cases the possible $z$ can be described explicitly as follows. 

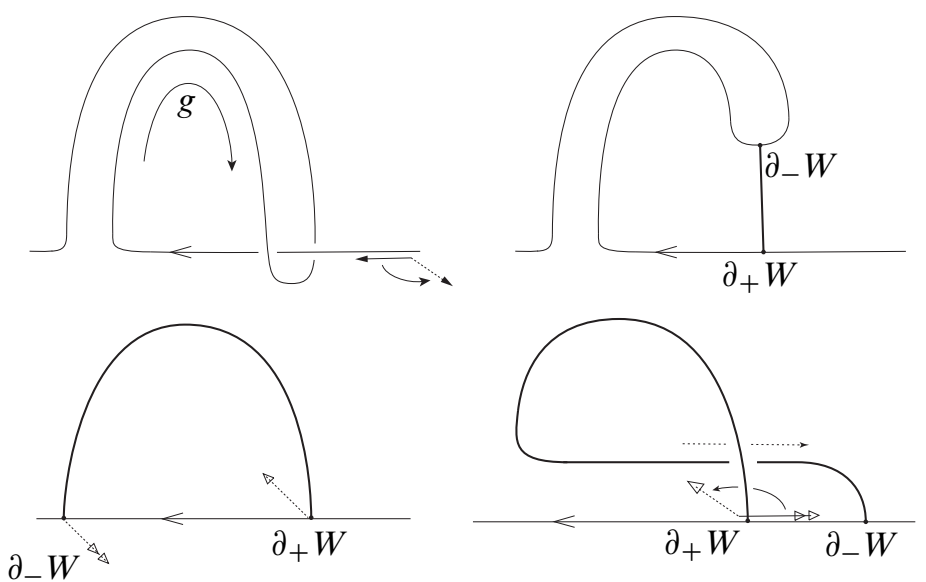

Figure 25: This figure describes a homotopy $A$ from the clasp knot $K$ shown in the upper left to the clasp knot $K^{\prime}$ which is determined by the guiding arc shown in the lower right. The undoing of the positive clasp creates a positive self-intersection in $A$, and the redoing of the clasp creates a canceling negative self-intersection. These self-intersections are paired by a framed Whitney disk $W$ (described by the trace of the guiding arc) whose boundary arcs $\partial_{+} W$ and $\partial_{+} W$ intersect in a single point $p$ where $\partial_{-} W$ pushes across $\partial_{+} W$ just before the lower right picture as indicated by the horizontal dotted arrow. The dotted vectors pointing lower-right point in the direction of increasing time, and the dotted vectors pointing upper-left point into the past. The orientation of $A$ is given by a tangent vector to $K$, together with a second tangent vector pointing into the future. The Whitney disk boundary arc $\partial_{+} W$ is oriented towards the positive selfintersection, and the boundary arc $\partial_{-} W$ is oriented towards the negative selfintersection. At $p$ the orientation of $A$ agrees with $\left(\partial_{-} W, \partial_{+} W\right)_{p}$. Using the assignment of trees to intersections between Whitney disk boundaries given by the formula $\epsilon_{p} \cdot t_{p}:=\epsilon_{k} \epsilon_{j}\left(1, g_{k}^{\epsilon_{k}}, g_{j}^{\epsilon_{j}}\right)$ in the "independence of Whitney disk boundaries" discussion in Section 2.4, and computing from the figure gives $\tau_{1}\left(K, K^{\prime}\right)=\tau_{1}(A)=-\left(g, g^{-1}\right)=+\left(g^{-1}, g\right)$, in the notation $(g, h):=(1, g, h)$.

5.2.1 The twisted circle bundle over the Möbius band In the first case, we use the notation

$$
\pi_{1}\left(B \tilde{\times} S^{1}\right)=\left\langle a, f \mid a f a^{-1}=f^{-1}\right\rangle<\pi_{1} M
$$

with $f$ represented by a circle fiber, $a$ represented by a core of the Möbius band $B$, and normal form $a^{n} f^{l}$ for $n, l \in \mathbb{Z}$. It is easily checked that the only candidates to be twisted stabilizers are $\psi=a^{2 r+1} f^{s}$, with $\psi^{2} \in\left\langle a^{2 k}\right\rangle$ central. For all $r, s \in \mathbb{Z}$, the 
orbits under conjugation by $a^{2 r+1} f^{s}$ are

$$
\left\{a^{2 k}\right\} \quad\left\{f^{l}, f^{-l}\right\} \quad\left\{a^{2 k} f^{l}, a^{2 k} f^{-l}\right\}
$$

for all nonzero integers $k$ and $l$; and

$$
\left\{a^{2 k+1} f^{l}, a^{2 k+1} f^{2 s-l}\right\} \quad\left\{a^{2 k+1}, a^{2 k+1} f^{2 s}\right\} \quad\left\{a^{2 k+1} f^{s}\right\}
$$

for all integers $k$ and $l$ with $l \neq s$. So in this case $\Psi_{z}$ is twisted exactly when $z$ is of the form

$$
\begin{aligned}
& \sum_{l \neq 0, k \neq 0} e_{k l}\left(a^{2 k} f^{l}+a^{2 k} f^{-l}\right)+e_{l} f^{l}+e_{k}^{\prime} a^{2 k} \\
& \quad+\sum_{l \neq s} d_{k l}\left(a^{2 k+1} f^{l}+a^{2 k+1} f^{2 s-l}\right)+d_{k}\left(a^{2 k+1}+a^{2 k+1} f^{2 s}\right)+d_{k}^{\prime} a^{2 k+1} f^{s},
\end{aligned}
$$

where the coefficients $e_{k l}, e_{l}, e_{k}^{\prime}, d_{k l}, d_{k}, d_{k}^{\prime}$ are integers, with some coefficient other than the $e_{k}^{\prime}$ nonzero.

We will show below in Section 5.3 that there is a self-homotopy $A$ of a knot $K$ having a latitude determining $a \in \Psi_{z}$ with $\tau_{0}(K)=z=f+f^{3}$ such that $\tau_{1}(A) \neq 0 \in$ $\mathcal{T}_{1}\left(\pi_{1} M\right) / \operatorname{INT}(z)$. This illustrates how Lemma 4.8 .3 (hence the proof of Theorem 3 ) can fail if $\Psi_{z}$ is twisted.

On the other hand, related examples will show that for $K$ with $\tau_{0}(K)=z=e_{l} f^{l}$, all self-homotopies $A$ of $K$ have $\tau_{1}(A)=0 \in \mathcal{T}_{1}\left(\pi_{1} M\right) / \operatorname{INT}(z)$. Thus, a twisted $\Psi_{z}$ does not guarantee the failure of Lemma 4.8.3, and so Theorem 3 can be extended to allow some twisted stabilizers.

5.2.2 Singular Seifert fibers In the case where $\psi$ is carried by a neighborhood of singular fiber in $N$ and $\left\{g_{i}\right\}$ lie in the canonical subgroup of $\pi_{1} N<\pi_{1} M$, we have $\psi=\phi^{r} f^{s}$ for $r, s \in \mathbb{Z}$ where $f$ is represented by a regular fiber of $N$ and $\phi$ is represented by a singular fiber of $N$. In this case $n \in \mathbb{N}$ is the smallest power such that $\phi^{r n}$ is contained in the cyclic (normal) subgroup $\langle f\rangle$ of $\pi_{1} N$. We get fixed $z$ of the form

$$
\sum e_{k}\left(g_{k}+\phi^{r} g_{k} \phi^{-r}+\phi^{2 r} g_{k} \phi^{-2 r}+\cdots+\phi^{(n-1) r} g_{k} \phi^{-(n-1) r}\right)+e_{p q} \phi^{p} f^{q}
$$

where the $\phi^{p} f^{q}$ lie in $\zeta\left(\phi^{r} f^{s}\right)$, so at least one $e_{k}$ must be nonzero.

5.2.3 Twisted $\Psi_{z}$ for links Now let $L$ be a link with $\tau_{0}(L)=z=\sum_{i \leq j} z_{i j}$, with each $z_{i j}$ written in normal form:

$$
z_{i j}=\sum_{p} e_{p}\left(g_{p}\right)_{i j}
$$


A twisted $\psi \in \Psi_{z}$ induces a nontrivial permutation of at least one of the sets $\left\{g_{p}^{ \pm 1}\right\}$ for $i=j$ or $\left\{g_{p}\right\}$ for $i<j$.

In the cases $i=j$ the above discussion for knots applies to describe restrictions on $z_{i i}$ and $\psi_{i}$ for twisted $\psi \in \Psi_{z}$. (Of course these restrictions are not independent of each other in the presence of nonzero $z_{i j}$ with $i<j$.)

Considering the case where $i<j$ and $\psi$ induces a nontrivial permutation of $\left\{g_{p}\right\}$ (twisting $z_{i j}$ ), there exists $n \geq 2$ such that $\psi_{i}^{n} g_{p} \psi_{j}^{-n}=g_{p}$ for all $g_{p}$. Thus $g_{p}^{-1} \psi_{i}^{n} g_{p}=\psi_{j}^{n}$, and $g_{p} \psi_{j}^{n} g_{p}^{-1}=\psi_{i}^{n}$ for all $g_{p}$. It follows from Proposition 4.5 of [19] that $\psi_{i}$ and $\psi_{j}$ are carried by singular fibers in a characteristic component $N \subset M$.

A more detailed analysis of twisted stabilizers would be interesting, but we stop here and conclude:

Proposition 5.2.1 If $M$ contains no orientable circle bundles over nonorientable surfaces and no Seifert fibered submanifolds having singular fibers, then $\Psi_{z}$ is untwisted for all $z \in \mathcal{T}_{0}\left(\pi_{1} M\right)$.

By direct computation one can extend Lemma 4.8.3 (hence Theorem 3) to certain simple examples of $z$ with twisted $\psi \in \Psi_{z}$ carried by a singular Seifert fiber, and it is possible that twisted $\Psi_{z}$ which correspond only to singular Seifert fibers do not contribute any nontrivial indeterminacies to $\tau_{1}\left(L_{z}, \cdot\right)$, but I do not know of a general construction. The next example shows that Lemma 4.8.3 can indeed fail for twisted $\Psi_{z}$ which correspond to orientable circle bundles over nonorientable surfaces.

\subsection{Knots in the twisted circle bundle over a Möbius band}

Using the notation of Section 5.2.1 above, this example will consider knots $K$ in $B \widetilde{\times} S^{1}$ with $\tau_{0}(K)=z=\sum e_{l} f^{l}$ carried by the circle factor. Such $z$ has twisted $\Psi_{z}$, with the conjugation action of $a$ (carried by the Möbius band core) inverting the $f^{l}$. After giving an example of a self-homotopy $A$ of such a $K$ with nontrivial $\tau_{1}(A)$ (so that Lemma 4.8.3 fails), we will observe that in some cases Lemma 4.8.3 can be extended to $K$ with twisted $\Psi_{z}$.

Throughout this subsection $(g, h):=(1, g, h)$ with the common univalent labels suppressed.

Since $B \widetilde{\times} S^{1}$ is irreducible, the $\Phi(z)$ relations are trivial throughout this subsection. 
5.3.1 A self-homotopy with nontrivial $\tau_{1}$ Illustrated in Figure 26, using the unknotplus-guiding-arcs notation, is a self-homotopy $A$ of a clasp knot $K$ in $B \widetilde{\times} S^{1}$, with $\tau_{0}(K)=z=f+f^{3}$. The right-back and left-front faces of the rectangular prism are identified by a half-rotation, and the top and bottom faces are identified by a translation, forming $B \widetilde{\times} S^{1}$. The clasp knot $K$ is described by the left-most rectangular unknot $U$ together with two guiding arcs, one which wraps once around the circle fiber and a longer one which wraps around three times. The self-homotopy $A$ pushes $K$ along the length of the Möbius band, so $A$ has a latitude representing the element $a \in \pi_{1} B \widetilde{\times} S^{1}$. During the description of $A$, the guiding arcs can be thought of either as representing the "bands" of $K$, or as tracing out a pair of Whitney disks in an order 1 Whitney tower on $A$.

As explained in the caption of Figure 26, $\tau_{1}(A)=(f, f)+\left(f^{-1}, f\right)+\left(f^{3}, f^{3}\right)+$ $\left(f^{-3}, f^{3}\right)$, which we will show is nonzero in $\mathcal{T}_{1}\left(\pi_{1} B \widetilde{\times} S^{1}\right) / \operatorname{INT}(z)$.

Noting that the edge decorations in $\tau_{1}(A)$ are carried by circle fibers, the relevant $\operatorname{INT}(z)$ relations for $z=f+f^{3}$ are

$$
0=\left(z+\bar{z}, f^{r}\right)=\left(f, f^{r}\right)+\left(f^{-1}, f^{r}\right)+\left(f^{3}, f^{r}\right)+\left(f^{-3}, f^{r}\right) \quad \text { for } r \in \mathbb{Z} .
$$

Denoting these relations by $\Delta_{r}$ and writing them in normal form $\left(f^{n}, f^{m}\right)$ with $0<n<m$ or $0=n \leq m$, we have

$$
\begin{array}{rlrl}
\Delta_{r} & =\left(f, f^{r}\right)-\left(f, f^{r+1}\right)+\left(f^{3}, f^{r}\right)-\left(f^{3}, f^{r+3}\right) & \text { for } 3<r \\
\Delta_{-r} & =\left(f^{r}, f^{r+1}\right)-\left(f^{r-1}, f^{r}\right)+\left(f^{r}, f^{r+3}\right)-\left(f^{r-3}, f^{r}\right) & & \text { for }-r \leq-3 \\
\Delta_{3} & =\left(f, f^{3}\right)-\left(f, f^{4}\right)+\left(1, f^{3}\right)-\left(f^{3}, f^{6}\right) & & \\
\Delta_{2} & =\left(f, f^{2}\right)-\left(f, f^{3}\right)-\left(f^{2}, f^{3}\right)-\left(f^{3}, f^{5}\right) & \\
\Delta_{1} & =(1, f)-\left(f, f^{2}\right)-\left(f, f^{3}\right)-\left(f^{3}, f^{4}\right) & \\
\Delta_{-1} & =\left(f, f^{2}\right)+(1, f)+\left(1, f^{4}\right)+\left(f^{2}, f^{3}\right) & \\
\Delta_{-2} & =\left(f^{2}, f^{3}\right)-\left(f, f^{2}\right)+\left(f^{2}, f^{5}\right)+\left(f, f^{3}\right) &
\end{array}
$$

with $\Delta_{0}$ already zero by the AS and FR relations.

We will show that $\tau_{1}(A)$ is not equal to a finite sum $\sum d_{i} \Delta_{i}$ in $\mathcal{T}_{1}\left(\pi_{1} B \tilde{\times} S^{1}\right)$ :

In normal form we have $\tau_{1}(A)=(1, f)-\left(f, f^{2}\right)+\left(1, f^{3}\right)-\left(f^{3}, f^{6}\right)$. Assume that $(1, f)-\left(f, f^{2}\right)+\left(1, f^{3}\right)-\left(f^{3}, f^{6}\right)=\sum d_{i} \Delta_{i}$. Note that for all $5 \leq r$ the coefficient of $\left(f, f^{r}\right)$ must vanish, so $d_{r-1}+d_{r}=0$. Thus, if $d_{4}$ is odd, then $\sum d_{i} \Delta_{i}$ can not be a finite sum. Similarly, for all $5 \leq r$ the coefficient of $\left(f^{r-1}, f^{r}\right)$ must vanish, so $d_{-(r-1)}+d_{-r}=0$. Thus, if $d_{-4}$ is odd, then $\sum d_{i} \Delta_{i}$ can not be a finite sum. 


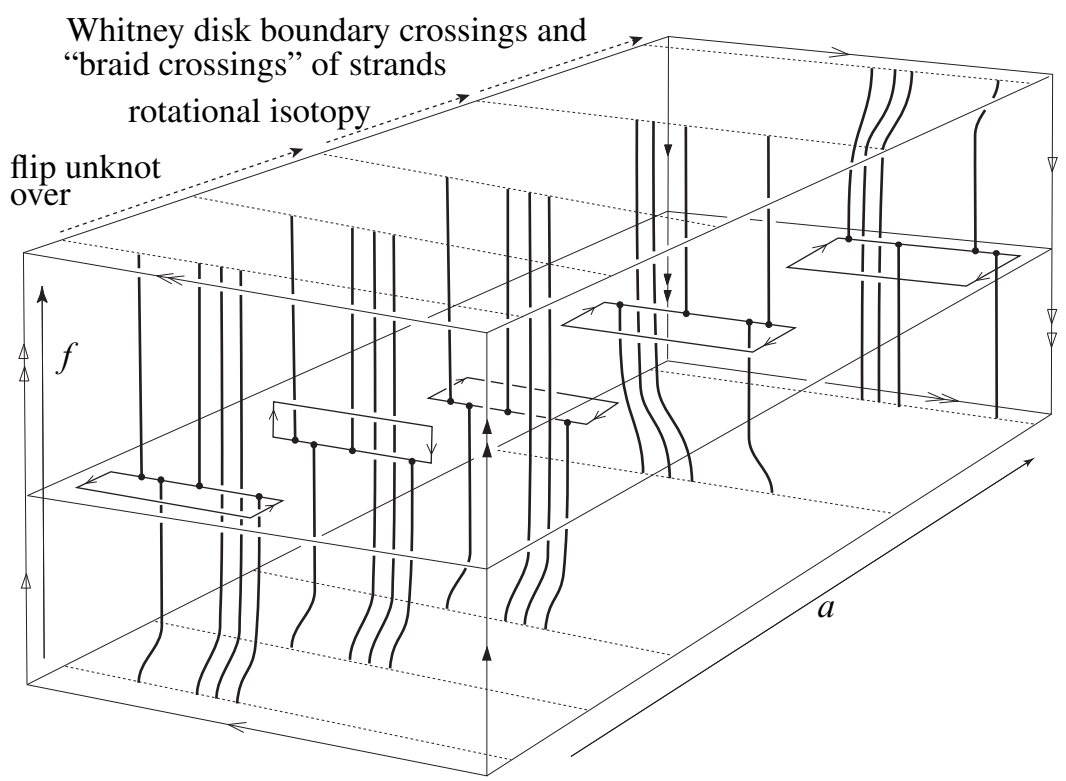

Figure 26: From left to right are pictured five stages of the self-homotopy $A$ of $K$ in the twisted circle bundle $B \widetilde{\times} S^{1}$ over the Möbius band. During the first three stages, the unknot $U$ flips over while fixing its edge that contains the endpoints of the guiding arcs. Between the second and third stages $U$ intersects each guiding arc near one endpoint - contributing $(f, f)+\left(f^{3}, f^{3}\right)$ to $\tau_{1}(A)$ - and intersects two strands of the longer guiding arc in canceling pairs of intersections which contribute nothing to $\tau_{1}(A)$. From the third stage to the fourth stage is just an ambient isotopy of $U$ together with the guiding arcs which is rotation by $180^{\circ}$ in the horizontal plane. From the fourth stage to the fifth stage each guiding arc pushes one of its endpoints across the other - contributing $\left(f^{-1}, f\right)+\left(f^{-3}, f^{3}\right)$ to $\tau_{1}(A)$, as illustrated in Figure 25 - and the vertical strands of the longer guiding arc pass through each other contributing nothing to $\tau_{1}(A)$. From the fifth stage back to the first stage is just an isotopy across the back face and in from the front face. Thus $\tau_{1}(A)=(f, f)+\left(f^{-1}, f\right)+\left(f^{3}, f^{3}\right)+\left(f^{-3}, f^{3}\right)$, which is shown above to be nonzero in $\mathcal{T}_{1}\left(\pi_{1} B \widetilde{\times} S^{1}\right) / \operatorname{INT}(z)$ (even modulo 2 , in case you don't want to check signs).

Working modulo 2 , the first three terms in $\tau_{1}(A)$ give the following relations among the coefficients:

$$
\begin{array}{ll}
(1, f): & d_{1}+d_{-1} \equiv 1 \\
\left(f, f^{2}\right): & d_{2}+d_{-2} \equiv 0 \\
\left(1, f^{3}\right): & d_{3}+d_{-3} \equiv 1 .
\end{array}
$$


The vanishing of the coefficient of $\left(f, f^{3}\right)$ gives

$$
d_{1}+d_{2}+d_{-2}+d_{3} \equiv 0
$$

which implies that $d_{1} \equiv d_{3}$, since $d_{2}+d_{-2} \equiv 0$. Now the vanishing of the coefficient of $\left(f^{3}, f^{4}\right)$, together with the congruence $d_{1} \equiv d_{3}$, give

$$
0 \equiv d_{1}+d_{-3}+d_{4}+d_{-4} \equiv d_{3}+d_{-3}+d_{4}+d_{-4} \equiv 1+d_{4}+d_{-4},
$$

so one of $d_{4}$ or $d_{-4}$ is odd, and $\sum d_{i} \Delta_{i}$ can not be a finite sum.

5.3.2 Extending Lemma 4.8.3 to some twisted stabilizers The construction of the self-homotopy $A$ in Figure 26 generalizes to clasp knots $K$ with $\tau_{0}(K)=\sum e_{l} f^{l}$, yielding self-homotopies $A$ with latitudes representing $a$, and

$$
\tau_{1}(A)=\sum e_{l}\left(\left(f^{l}, f^{l}\right)+\left(f^{-l}, f^{l}\right)\right) .
$$

In the case where $\tau_{0}(K)=z=e_{l} f^{l}$, it is clear that $\tau_{1}(A)=e_{l}\left(\left(f^{l}, f^{l}\right)+\left(f^{-l}, f^{l}\right)\right) \in$ INT $(z)$ by taking $r=l$ in the INT relations, and Lemma 4.8.3 can be extended to such $K$. (In the case $e_{l}=1$ so $K$ has just a single clasp then $A$ can be taken to be a self-isotopy of $K$.) Notice that composing $A$ with itself in the general case gives a self-homotopy $A+A$ of $K$ with a latitude representing $a^{2}$ which is untwisted, and the computation

$$
\begin{aligned}
\tau_{1}(A+A) & =\tau_{1}(A)+a \cdot \tau_{1}(A) \\
& =\sum e_{l}\left(\left(f^{l}, f^{l}\right)+\left(f^{-l}, f^{l}\right)\right. \\
& \left.\quad+\left(a f^{l} a^{-1}, a f^{l} a^{-1}\right)+\left(a f^{-l} a^{-1}, a f^{l} a^{-1}\right)\right) \\
& =\sum e_{l}\left(\left(f^{l}, f^{l}\right)+\left(f^{-l}, f^{l}\right)+\left(f^{-l}, f^{-l}\right)+\left(f^{l}, f^{-l}\right)\right) \\
& =0 \in \mathcal{T}_{1}\left(\pi_{1} B \tilde{\times} S^{1}\right)
\end{aligned}
$$

confirms Lemma 4.8 .3 (using $\left(f^{-l}, f^{-l}\right)+\left(f^{l}, f^{l}\right)=0$ and $\left(f^{l}, f^{-l}\right)+\left(f^{-l}, f^{l}\right)=$ 0 , by HOL, FR and AS relations).

It would be useful to find general techniques for computing in the infinitely generated groups $\mathcal{T}_{1}\left(\pi_{1} B \tilde{\times} S^{1}\right) / \operatorname{INT}(z)$.

\section{Essential knots}

The goal of this section is Theorem 5 (Section 6.4.2) which, together with Theorem 3 in the introduction, classifies stable knot concordance in products $F \times S^{1}$ of an orientable 
surface $F \neq S^{2}$ with the circle. Most of the work involves characterizing new INT relations which arise when defining $\tau_{1}$ for singular concordances of knots which are not null-homotopic.

Throughout this section we continue to assume that $M$ is an oriented 3-manifold, with $\pi_{1} M$ torsion-free, and add the further assumption that $M$ is irreducible. We restrict our attention to knots in such $M$, eventually focusing on the case where $M=F \times S^{1}$. Consider an essential knot $K \subset M$ with $[K]=\gamma \neq 1$ generating a cyclic subgroup $\langle\gamma\rangle<\pi_{1} M$, for some choice of whisker on $K$. For any singular concordance $A \rightarrow \rightarrow$ $M \times I$ of $K$ which supports an order 1 Whitney tower $\mathcal{W}$, we want to define $\tau_{1}(A)$ as before by associating decorated Y-trees to the intersections between $A$ and the interiors of the Whitney disks in $\mathcal{W}$. Defining $\tau_{1}$ for such an essential annulus will require two modifications of the previous definition for inessential annuli: The edge decorations will now be cosets by $\langle\gamma\rangle$, to account for choices of paths in $A$; and a reformulation of the $\operatorname{INT}(A)$ relations will account for new indeterminacies in the choices of Whitney disk boundaries. The relevant $\operatorname{INT}(A)$ relations will turn out to be determined by order zero intersections between singular self-concordances which have latitudes representing generators of the centralizer $\zeta(\gamma)$ of $\gamma$ in $\pi_{1} M$.

\subsection{Order zero double coset decorations}

We start by discussing the order zero intersection invariants that will be used to define the new INT relations and index the equivalence classes of singular concordances which support an order 1 Whitney tower.

Define the abelian group $\mathcal{T}_{0}\left(\langle\gamma\rangle \backslash \pi_{1} M /\langle\gamma\rangle\right)$ in the same way as $\mathcal{T}_{0}\left(\pi_{1} M\right)$ except that the order zero trees are decorated by (representatives of) double cosets of $\pi_{1} M$ by $\langle\gamma\rangle$.

In defining an order zero intersection invariant for annuli, we will only be concerned with two separate cases: either all univalent vertices will have the same label; or the pairs of univalent vertices on all edges (order zero trees) will be labeled distinctly from a set of two labels. So in the latter case $\mathcal{T}_{0}\left(\langle\gamma\rangle \backslash \pi_{1} M /\langle\gamma\rangle\right)$ can be identified with the $\mathbb{Z}$-span of the double coset space, and in the first case one takes a further quotient by inversion of coset representatives and by killing the double coset represented by the trivial element $1 \in \pi_{1} M$.

If $A$ and $A^{\prime}$ are singular concordances of knots in $M$ representing $\gamma$, then sheetchanging paths through the intersections between $A$ and $A^{\prime}$ determine double cosets, and we define the order zero nonrepeating intersection tree

$$
\lambda_{0}\left(A, A^{\prime}\right) \in \mathcal{T}_{0}\left(\langle\gamma\rangle \backslash \pi_{1} M /\langle\gamma\rangle\right)
$$


by summing as usual over the signed intersections between $A$ and $A^{\prime}$, and ignoring any self-intersections. $\lambda_{0}\left(A, A^{\prime}\right)$ is invariant under homotopy (rel $\partial$ ) and is the complete obstruction to pairing $A \cap A^{\prime}$ by Whitney disks (an order 1 nonrepeating Whitney tower).

Similarly, the order zero self-intersection tree

$$
\tau_{0}(A) \in \mathcal{T}_{0}\left(\langle\gamma\rangle \backslash \pi_{1} M /\langle\gamma\rangle\right)
$$

is defined by summing over all signed self-intersections of $A$, is invariant under homotopy (rel $\partial$ ), and is the complete obstruction to the existence of an order 1 Whitney tower on $A$.

\subsection{Order 1 coset decorations}

Define the abelian group $\mathcal{T}_{1}\left(\langle\gamma\rangle \backslash \pi_{1} M\right)$ in the same way as $\mathcal{T}_{1}\left(\pi_{1} M\right)$ except that the edges of the Y-tree generators are decorated by (representatives of) left cosets $\langle\gamma\rangle \backslash \pi_{1} M$ - here we are assuming that all edges in our $Y$-trees are oriented towards the trivalent vertex. In this section we only consider knots, so all univalent vertices in $\mathcal{T}_{1}\left(\langle\gamma\rangle \backslash \pi_{1} M\right)$ have the same label which is suppressed from notation.

$\mathcal{T}_{1}\left(\langle\gamma\rangle \backslash \pi_{1} M\right)$ is the quotient of $\mathcal{T}_{1}\left(\pi_{1} M\right)$ by the relations:

$$
(a, b, c)=\left(\gamma^{q} a, \gamma^{r} b, \gamma^{s} c\right) \quad \text { for } \quad q, r, s \in \mathbb{Z} .
$$

For an order 1 Whitney tower $\mathcal{W}$ on a singular concordance $A$ of $K$ as above, it is clear that these relations are exactly what is needed to account for choices of sheet-changing paths from $A$ into the Whitney disks $\mathcal{W}$ when defining $\tau_{1}(A)$.

\subsection{INT relations for essential knots}

Fixing the boundary and changing the interior of any Whitney disk in $\mathcal{W}$ does not change $\tau_{1}(\mathcal{W})$ since we are assuming that $M$ is irreducible. Also, by the same argument as in Section 2.4, changing the boundaries of the Whitney disks in $\mathcal{W}$ by a regular homotopy in $A$ does not change $\tau_{1}(\mathcal{W})$. However, changing the homotopy class in $A$ of Whitney disk boundaries can change $\tau_{1}(\mathcal{W})$ as we discuss next.

Consider a Whitney disk $W$ in $\mathcal{W}$. Changing $\partial W$ by winding one arc $m$ times around $A$ and the other arc $n$ times around $A$ creates a closed curve in $A$ representing $\gamma^{m} \beta \gamma^{n} \beta^{-1} \in \pi_{1} M$, where $\beta$ is determined by double-point loops through points paired by $W$, and the signs of the integers $m$ and $n$ correspond to the direction of winding relative to $\gamma$. This closed curve bounds a Whitney disk exactly when $\gamma^{m} \beta \gamma^{n} \beta^{-1}=1$. Solutions to such Baumslag-Solitar equations are highly restricted in 
3-manifold groups, for instance if $M$ is sufficiently large then $m= \pm n$ by Jaco [18]. We make the further assumption that if $\gamma^{m} \beta \gamma^{n} \beta^{-1}=1$, then $\gamma$ and $\beta$ commute, as will be the case for all $\gamma$ when $M=F \times S^{1}$.

Assuming that $\gamma^{m} \beta \gamma^{n} \beta^{-1}=1$ implies $\gamma \beta \gamma^{-1} \beta^{-1}=1$, it is sufficient to compute the change in $\tau_{1}(\mathcal{W})$ due to changing a Whitney disk boundary $\partial W$ by winding one arc around $A$ in the positive $\gamma$ direction and the other arc around $A$ in the direction of $\gamma^{-1}$, and replacing $W$ by a Whitney disk $W^{\prime}$ with this new boundary. Such a change is illustrated in the left hand side of Figure 27, which also indicates how two thin bands (half-tubes) $B$ and $C$ can be added to $W$ so that $W \cup B \cup C \cup W^{\prime}$ forms a torus $T$ (here the orientation of $W^{\prime}$ is reversed). Since $A$ can be assumed to miss $B$ and $C$, the order zero intersections between $A$ and $T$ measure the change in $\tau_{1}$ as follows.
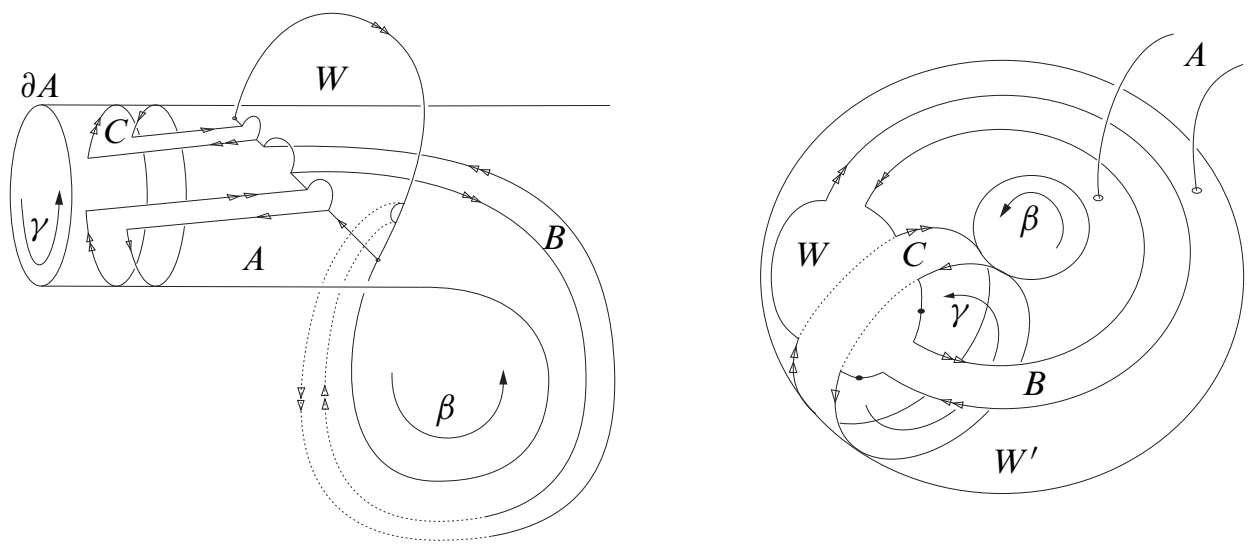

Figure 27

Take a whisker on $T$ to be a common whisker for $W$ and $W^{\prime}$ where their boundaries meet. As in the previous INT relations, the two descending edges on Y-trees for $W$ and $W^{\prime}$ are decorated by the same pair of fundamental group elements (now coset representatives), which here can be normalized to 1 and $\beta$ by choosing the whisker on $T$ appropriately. The change in $\tau_{1}$ is determined by sheet-changing paths from $A$ into $T$ which represent cosets decorating the third edges of the Y-trees. Note that allowing these paths to cross $B$ is irrelevant since we are working modulo multiplication by $\gamma$, but we can not assume to allow paths in $T$ to cross $C$ which would correspond to multiplication by $\beta$. So the change in $\tau_{1}$ is truly measured by order zero intersections between $A$ and the annulus which is gotten from $T$ essentially by cutting out $C$, as described next.

The band $C$ can be extended to an annulus $\bar{C}$ by adding a small embedded band in $W$, as indicated by the dotted lines in the right-hand side of Figure 27. Remove $\bar{C}$ 
from $T$ and extend the resulting boundary components by disjointly embedded collars to parallel copies of $K$ in $M \times\{0\}$ to get an annulus $A^{\prime}$ which has the same order zero intersections with $A$ as $T$. Now the change in $\tau_{1}$ can be expressed as $\left(1, \beta, \lambda_{0}\left(A, A^{\prime}\right)\right)$, where $\lambda_{0}$ is the order zero nonrepeating intersection tree of Section 6.1 above. Since $A$ has only canceling pairs of self-intersections, we can add a parallel copy of $A$ to $A^{\prime}$ along one boundary component to get a singular concordance $A^{\prime \prime}$ of a parallel copy of $K$ such that $\lambda_{0}\left(A, A^{\prime \prime}\right)=\lambda_{0}\left(A, A^{\prime}\right)$. Now we can extend both $A$ and $A^{\prime \prime}$ by $-A$ (and a parallel copy) to get pair of singular self-concordances $A_{1}$ and $A_{2}$ of $K$ and a parallel copy of $K$. Again, the intersections between $-A$ and its parallel copy occur in canceling pairs, so the change in $\tau_{1}$ can be expressed as $\left(1, \beta, \lambda_{0}\left(A_{1}, A_{2}\right)\right)$.

By Lemma 4.8.1 and the homotopy invariance of $\lambda_{0}, \lambda_{0}\left(A_{1}, A_{2}\right)$ only depends on the elements determined by latitudes of $A_{1}$ and $A_{2}$. Since a latitude of $A_{1}=A-$ $A$ determines the trivial element $1 \in \pi_{1} M, A_{1}$ is homotopic to the product selfconcordance $K \times I$, also by Lemma 4.8.1. Since $A^{\prime}$ has a latitude representing $\beta$, and $A_{2}$ was formed by extending $A^{\prime}$ by $A-A$, a latitude of $A_{2}$ determines the element $\beta$ in the centralizer $\zeta(\gamma)$ of $\gamma$ in $\pi_{1} M$. Thus, the possible changes in $\tau_{1}(\mathcal{W})$ are measured by $\lambda_{0}\left(K \times I, A_{\beta}\right)$ where $\beta$ ranges over $\zeta(\gamma)$, and we have the following INT relations.

For $[K]=\gamma \in \pi_{1} M$, define the $\operatorname{INT}(K)$ relations by

$$
\operatorname{INT}(K)=\left(1, \beta, \lambda_{0}\left(K \times I, A_{\beta}\right)\right)
$$

where $\beta$ ranges over $\zeta(\gamma)$ and $A_{\beta}$ is any singular self-concordance of $K$ having a latitude representing $\beta$. These INT relations are completely determined by the values on a set of generators for $\zeta(\gamma)$ as illustrated in the next subsection.

It follows from this discussion that (assuming $\gamma$ has only trivial Baumslag-Solitar solutions) for any singular concordance $A$ of $K$ supporting an order 1 Whitney tower $\mathcal{W}$

$$
\tau_{1}(A):=\tau_{1}(\mathcal{W}) \in \frac{\mathcal{T}_{1}\left(\langle\gamma\rangle \backslash \pi_{1} M\right)}{\operatorname{INT}(K)}
$$

does not depend on the choice of $\mathcal{W}$, and is invariant under regular homotopy (rel $\partial$ ) of $A$. Also, the geometric conditions equivalent to the vanishing of previous versions of $\tau_{1}$ hold in this setting since again the relations can all be realized by geometric constructions.

\subsection{Stable concordance of knots in $F \times S^{1}$}

We now restrict attention to essential knots in $M=F \times S^{1}$, the product of a compact orientable surface $F \neq S^{2}$ with the circle. The results in this section, together with 
Theorem 3 for null-homotopic knots, give a complete characterization of stable knot concordance in this setting.

6.4.1 The order zero relative self-linking invariant Fix a knot $K_{\gamma}$ in a nontrivial free homotopy class together with a whisker such that $\left[K_{\gamma}\right]=\gamma \in \pi_{1} M$. For any singular concordance $A$ from $K_{\gamma}$ to any knot $K$, define the order zero relative selfintersection tree $\tau_{0}\left(K_{\gamma}, K\right)$ by

$$
\tau_{0}\left(K_{\gamma}, K\right):=\tau_{0}(A) \in \mathcal{T}_{0}\left(\langle\gamma\rangle \backslash \pi_{1} M /\langle\gamma\rangle\right) .
$$

By [28], it is possible to choose $K_{\gamma}$ in each free homotopy class so that the order zero relative self-linking with $K_{\gamma}$ classifies order 1 Whitney concordance, meaning that $\tau_{0}\left(K_{\gamma}, K\right)=\tau_{0}\left(K_{\gamma}, K^{\prime}\right)$ if and only if $K$ and $K^{\prime}$ are order 1 Whitney concordant, for any knots $K$ and $K^{\prime}$ (freely) homotopic to $K_{\gamma}$. (This invariant was denoted $\mu\left(K_{\gamma}, K\right)$ in [28].) Here equality is modulo conjugation by the centralizer $\zeta(\gamma)$, ie modulo the restriction to $\zeta(\gamma)$ of the $\Psi$-action on $\mathcal{T}_{0}\left(\langle\gamma\rangle \backslash \pi_{1} M /\langle\gamma\rangle\right)$. Note that choices of whiskers on $K_{\gamma}$ giving $\left[K_{\gamma}\right]=\gamma$ differ by loops representing elements in $\zeta(\gamma)$, and all latitudes of singular self-concordances of $K_{\gamma}$ determine elements in $\zeta(\gamma)$.

As explained in [28], the key property required of $K_{\gamma}$ in order for $\tau_{0}\left(K_{\gamma}, \cdot\right)$ to classify order 1 Whitney concordance is that $\tau_{0}(H)=0 \in \mathcal{T}_{0}\left(\langle\gamma\rangle \backslash \pi_{1} M /\langle\gamma\rangle\right)$ for any singular self-concordance $H$ of $K_{\gamma}$. (In the present case $M$ is irreducible; in general the required vanishing of $\tau_{0}(H)$ would be modulo intersections with 2-spheres - hence the terminology " $K_{\gamma}$ is spherical" in [28].) This key property can be checked on self-homotopies having latitudes representing a set of generators for $\zeta(\gamma)$, and can be easily satisfied by choosing $K_{\gamma}$ as follows.

If $\gamma$ is carried by (a multiple of) a circle fiber, then take $K_{\gamma}$ to lie in a tubular neighborhood of a fiber. In this case, $\zeta(\gamma)=\pi_{1} M$, and $K_{\gamma}$ can be isotoped around any generating loop in $M$. If $\gamma$ is not carried by a circle fiber, then take $K_{\gamma}$ in a tubular neighborhood of a knot $K_{\rho}$ representing a primitive element $\rho \in \pi_{1} M$, with $\gamma=\rho^{n}$ for some natural number $n$. In this case $\zeta(\gamma)$ will be isomorphic to $\mathbb{Z} \oplus \mathbb{Z}$ generated by $\rho$ and a fiber element, unless $F$ is either a 2 -torus or 2-disk, and in all cases $K_{\gamma}$ can be isotoped around loops representing generators of $\zeta(\gamma)$.

6.4.2 The order 1 relative self-linking invariant Fix now $K_{\gamma}$ as above, which classifies order 1 Whitney concordance in a nontrivial free homotopy class represented by $\left[K_{\gamma}\right]=\gamma \in \pi_{1} M$. For any $z \in \mathcal{T}_{0}\left(\langle\gamma\rangle \backslash \pi_{1} M /\langle\gamma\rangle\right)$, construct a clasp knot $K_{z}$ such that $\tau_{0}\left(K_{\gamma}, K_{z}\right)=z$ by introducing clasps into $K_{\gamma}$ (just as was done for nullhomotopic knots). For any $K$ which is order 1 Whitney concordant to $K_{z}$ (equivalently 
$\left.\tau_{0}\left(K_{\gamma}, K\right)=z\right)$, define the order 1 relative self-intersection tree $\tau_{1}\left(K_{z}, K\right)$ by

$$
\tau_{1}\left(K_{z}, K\right):=\tau_{1}(A) \in \frac{\mathcal{T}_{1}\left(\langle\gamma\rangle \backslash \pi_{1} M\right)}{\operatorname{INT}(z)}
$$

where $A$ is any concordance from $K_{z}$ to $K$, and the $\operatorname{INT}(z)$ relations are

$$
\left(1, \beta^{r},\left(1-\beta^{r}\right)(z+\bar{z})\right)=0
$$

with $\beta$ ranging over a generating set for $\zeta(\gamma)$, and $r \in \mathbb{Z}$.

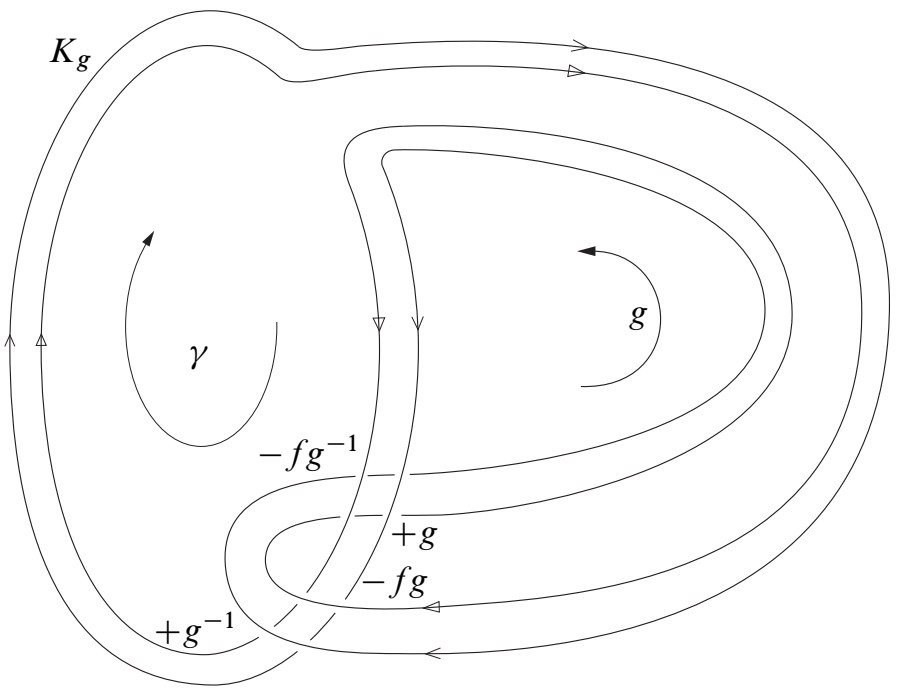

Figure 28: A knot $K_{g}$ together with a parallel copy sitting in a slice $F \times I$ of $F \times S^{1}$. A self-isotopy $A_{f}$ of the parallel copy up around the circle direction creates the indicated crossing-change intersections, giving $\lambda_{0}\left(K_{g} \times I, A_{f}\right)=$ $g+g^{-1}-f g-f g^{-1}$.

To see that these are the correct INT relations $\left(\operatorname{INT}(z)=\operatorname{INT}\left(K_{z}\right)\right.$ as defined above in Section 6.3) start by considering Figure 28, which illustrates the case where $\gamma$ is primitive and $z$ is represented by a single element $g \in \pi_{1}\left(F \times S^{1}\right) \cong \pi_{1} F \times\langle f\rangle$, with both $\gamma$ and $g$ contained in the subgroup $\pi_{1} F$ carried by a thickened surface slice which in the figure is represented by the plane of the paper cross an interval. Isotoping a parallel push-off of $K_{g}$ up around the circle direction describes an annulus $A_{f}$ which has a latitude representing the circle generator $f$, and the order zero nonrepeating intersection tree

$$
\lambda_{0}\left(K_{g} \times I, A_{f}\right)=g+g^{-1}-f g-f g^{-1}=(1-f)\left(g+g^{-1}\right)
$$


can be computed from crossing changes in the figure. Note that introducing twists between $K_{g}$ and its parallel push-off only creates terms of the form $\pm(1-f)$ via this construction which do not contribute to $\operatorname{INT}(g)$ because the 2-torsion elements $(1, f,(1-f))=(1, f, 1)-(1, f, f)$ are zero in $\mathcal{T}_{1}$ by the AS and FR relations.

Iterating this construction confirms the relations $\left(1, f^{r},\left(1-f^{r}\right)\left(g+g^{-1}\right)\right)$ for the generator $f \in \zeta(\gamma)$ in this case. It is easy to see that the same computation holds if $g$ replaced by any sum $z=\sum g_{i}$, even if $g_{i}$ are allowed to contain powers of $f$ as factors (with corresponding clasps guided by arcs wrapping around circle fibers). One can also check that allowing $\gamma$ to contain factors of $f$ does not change the computation.

Under the assumption that $\gamma$ is primitive, the computation so far verifies the formula for the $\operatorname{INT}(z)$ relations in the "generic" case where $\zeta(\gamma) \cong \mathbb{Z} \oplus \mathbb{Z}$ is generated by $f$ and $\gamma$, since in this case self-homotopies with latitudes representing $\gamma$ give only trivial relations: A parallel push-off of the product annulus $K_{z} \times I$ is disjoint from $K_{z} \times I$ and has a latitude representing $\gamma$.

Consider now the case where $\gamma=f$, so that $K_{z}$ is created by introducing clasps into a circle fiber $K_{f}$. In this case $f$ is central, and for any $\beta \in \pi_{1} M$, a self-homotopy $A_{\beta}$ of $K_{z}$ with a latitude representing $\beta$ and $\lambda_{0}\left(K_{z} \times I, A_{\beta}\right)=(1-\beta)(z+\bar{z})$ can be constructed as follows. Undoing the clasps on a parallel copy $K_{z}^{\prime}$ of $K_{z}$ describes a homotopy of $K_{z}^{\prime}$ to a knot $K_{f}^{\prime}$ which may twist around the circle fiber $K_{f}$. The homotopy so far creates intersections with $K_{z} \times I$ of the form $z+\bar{z}$ (half of the crossing changes in Figure 28 for each clasp). Next, $K_{f}^{\prime}$ can be isotoped around $M$ so that a basepoint on $K_{f}^{\prime}$ traces out a loop representing $\beta$; this self isotopy of $K_{f}^{\prime}$ only creates intersections with $K_{z} \times I$ of the form $\pm(1-\beta)$ corresponding to any twists between $K_{f}^{\prime}$ and $K_{f}$ which are pulled apart and then recreated; as mentioned above these intersections do not contribute to $\operatorname{INT}(z)$. Now reintroducing the clasps into $K_{f}^{\prime}$ describes the last part of the self-homotopy $A_{\beta}$ of $K_{z}$, and creates intersections of the form $-\beta(z+\bar{z})$, so that $\lambda_{0}\left(K_{z} \times I, A_{\beta}\right)=(1-\beta)(z+\bar{z})$ as desired.

The cases where $\gamma$ is not primitive are checked by constructing self-homotopies similar to the case $\gamma=f$ in the previous paragraph. For instance, if $\gamma=f^{n}$ then $K_{z}$ is created from $K_{f}$ which is contained in a tubular neighborhood of a circle fiber and the rest of the construction as for the case $n=1$ applies. The same construction applies for $\gamma=\rho^{n}$, with $\rho$ a primitive generator of $\zeta(\gamma)$ and $K_{z}$ created from $K_{\rho^{n}}$ which is contained in a tubular neighborhood of $K_{\rho}$; and also to any $\gamma$ in the nongeneric cases where $F$ is a torus or a disk.

Combined with Theorem 3, the following theorem gives a classification of stable knot concordance in $F \times S^{1}$ : 
Theorem 5 For $K_{z} \subset M=F \times S^{1}$ as above, the following are equivalent:

(i) $\tau_{1}\left(K_{z}, K\right)=\tau_{1}\left(K_{z}, K^{\prime}\right)$.

(ii) $K$ and $K^{\prime}$ are stably concordant.

(iii) $K$ and $K^{\prime}$ are order 2 Whitney concordant.

(iv) $K$ and $K^{\prime}$ are height 1 Whitney concordant.

Furthermore, for any such $K_{z}$ each element in the target group can be realized as $\tau_{1}\left(K_{z}, K\right)$ for some $K$.

Proof The proof of Theorem 5 follows the arguments in Sections 4.6 and 4.7 in the proof of Theorem 3. The key point needed is that $\tau_{1}$ vanishes on all self-homotopies of $K_{z}$ which support an order 1 Whitney tower (equivalently, have vanishing $\tau_{0}$ ). If $H_{\psi}$ is a self-homotopy of $K_{z}$ with a latitude representing $\psi$, then $\psi \in \zeta(\gamma)$, and by Lemma 4.8.1 $\tau_{0}\left(H_{\psi}\right)$ only depends on $\psi$. It follows that $\tau_{0}\left(H_{\psi}\right)=z-\psi z \psi^{-1}$, since such $H_{\psi}$ can be constructed by undoing the clasps on $K_{z}$ to get $K_{\gamma}$, then isotoping $K_{\gamma}$ around $\psi \in \zeta(\gamma)$, then redoing the clasps to return to $K_{z}$. Thus, such an $H_{\psi}$ admits an order 1 Whitney tower if and only if $\tau_{0}\left(H_{\psi}\right)$ vanishes if and only if $\psi \in \Psi_{z} \cap \zeta(\gamma)$. In the current setting, $\Psi_{z}$ is always untwisted, and it can be easily checked that for $\psi \in \Psi_{z} \cap \zeta(\gamma)$ the self-isotopies of $K_{\gamma}$ realizing $\psi$ (as described above in Section 6.4.1) extend to self-isotopies of $K_{z}$.

\section{References}

[1] C Bohr, Stabilisation, bordism and embedded spheres in 4-manifolds, Algebr. Geom. Topol. 2 (2002) 219-238 MR1917050

[2] S Boyer, D Rolfsen, B Wiest, Orderable 3-manifold groups, Ann. Inst. Fourier (Grenoble) 55 (2005) 243-288 MR2141698

[3] A Casson, D Jungreis, Convergence groups and Seifert fibered 3-manifolds, Invent. Math. 118 (1994) 441-456 MR1296353

[4] V Chernov, Framed knots in 3-manifolds and affine self-linking numbers, J. Knot Theory Ramifications 14 (2005) 791-818 MR2172898

[5] J Conant, R Schneiderman, P Teichner, Geometric filtrations of link concordance, in preparation

[6] J Conant, R Schneiderman, P Teichner, Link concordance invariants via Whitney towers, in preparation

[7] J Conant, R Schneiderman, P Teichner, Whitney towers and a conjecture of Levine, in preparation 
[8] J Conant, R Schneiderman, P Teichner, Jacobi identities in low-dimensional topology, Compos. Math. 143 (2007) 780-810 MR2330447

[9] M Freedman, R Kirby, A geometric proof of Rochlin's theorem, from: "Algebraic and geometric topology (Proc. Sympos. Pure Math., Stanford Univ., 1976), Part 2”, (R J Milgram, editor), Proc. Sympos. Pure Math. XXXII, Amer. Math. Soc. (1978) 85-97 MR520525

[10] M H Freedman, F Quinn, Topology of 4-manifolds, Princeton Math. Series 39, Princeton Univ. Press (1990) MR1201584

[11] D Gabai, Convergence groups are Fuchsian groups, Ann. of Math. (2) 136 (1992) 447-510 MR1189862

[12] S Garoufalidis, M Goussarov, M Polyak, Calculus of clovers and finite type invariants of 3-manifolds, Geom. Topol. 5 (2001) 75-108 MR1812435

[13] S Garoufalidis, J Levine, Homology surgery and invariants of 3-manifolds, Geom. Topol. 5 (2001) 551-578 MR1833753

[14] C H Giffen, Link concordance implies link homotopy, Math. Scand. 45 (1979) 243-254 MR580602

[15] D L Goldsmith, Concordance implies homotopy for classical links in $M^{3}$, Comment. Math. Helv. 54 (1979) 347-355 MR543335

[16] K Habiro, Claspers and finite type invariants of links, Geom. Topol. 4 (2000) 1-83 MR1735632

[17] A Hatcher, Notes on basic 3-manifold topology Available at http:// ww. math. cornell.edu/ hatcher

[18] W Jaco, Roots, relations and centralizers in three-manifold groups, from: "Geometric topology (Proc. Conf., Park City, Utah, 1974)”, (L C Glaser, T B Rushing, editors), Lecture Notes in Math. 438, Springer, Berlin (1975) 283-309 MR0394668

[19] W Jaco, P B Shalen, A new decomposition theorem for irreducible sufficiently-large 3-manifolds, from: "Algebraic and geometric topology (Proc. Sympos. Pure Math., Stanford Univ., 1976), Part 2”, (R J Milgram, editor), Proc. Sympos. Pure Math. XXXII, Amer. Math. Soc. (1978) 71-84 MR520524

[20] W H Jaco, P B Shalen, Seifert fibered spaces in 3-manifolds, Mem. Amer. Math. Soc. 21 (1979) viii+192 MR539411

[21] E Kalfagianni, Finite type invariants for knots in 3-manifolds, Topology 37 (1998) 673-707 MR1604915

[22] P Kirk, C Livingston, Type 1 knot invariants in 3-manifolds, Pacific J. Math. 183 (1998) 305-331 MR1625970

[23] P Kirk, C Livingston, Knot invariants in 3-manifolds and essential tori, Pacific J. Math. 197 (2001) 73-96 MR1810209 
[24] J P Levine, Surgery on links and the $\bar{\mu}$-invariants, Topology 26 (1987) 45-61 MR880507

[25] Y Matsumoto, Secondary intersectional properties of 4-manifolds and Whitney's trick, from: "Algebraic and geometric topology (Proc. Sympos. Pure Math., Stanford Univ., 1976), Part 2”, (R J Milgram, editor), Proc. Sympos. Pure Math. XXXII, Amer. Math. Soc. (1978) 99-107 MR520526

[26] R A Norman, Dehn's lemma for certain 4-manifolds, Invent. Math. 7 (1969) 143-147 MR0246309

[27] N Sato, Cobordisms of semiboundary links, Topology Appl. 18 (1984) 225-234 MR769293

[28] R Schneiderman, Algebraic linking numbers of knots in 3-manifolds, Algebr. Geom. Topol. 3 (2003) 921-968 MR2012959

[29] R Schneiderman, Simple Whitney towers, half-gropes and the Arf invariant of a knot, Pacific J. Math. 222 (2005) 169-184 MR2200249

[30] R Schneiderman, Whitney towers and gropes in 4-manifolds, Trans. Amer. Math. Soc. 358 (2006) 4251-4278 MR2231378

[31] R Schneiderman, P Teichner, Higher order intersection numbers of 2-spheres in 4-manifolds, Algebr. Geom. Topol. 1 (2001) 1-29 MR1790501

[32] R Schneiderman, P Teichner, Whitney towers and the Kontsevich integral, from: "Proceedings of the Casson Fest", (C Gordon, Y Rieck, editors), Geom. Topol. Monogr. 7 (2004) 101-134 MR2172480

[33] R Stong, Existence of $\pi_{1}$-negligible embeddings in 4-manifolds. A correction to Theorem 10.5 of Freedmann and Quinn, Proc. Amer. Math. Soc. 120 (1994) 1309-1314 MR1215031

Department of Mathematics and Computer Science, Lehman College City University of New York, New York, NY robert.schneiderman@lehman . cuny.edu http://comet. lehman. cuny.edu/schneiderman/

Received: 26 December 2008 Revised: 13 November 2009 